\title{
Botanicals and Their Bioactive Phytochemicals for Women's Health
}

\author{
Birgit M. Dietz, Atieh Hajirahimkhan, Tareisha L. Dunlap, and Judy L. Bolton
}

University of Illinois at Chicago/National Institutes of Health Center for Botanical Dietary Supplements, Department of Medicinal Chemistry and Pharmacognosy, College of Pharmacy, University of Illinois at Chicago, Chicago, Illinois

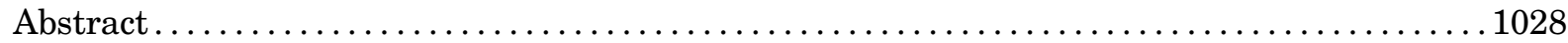

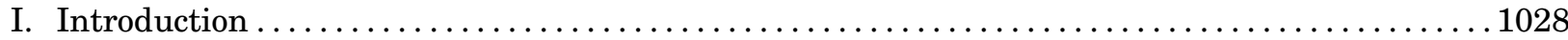

A. Women's Health as a Function of Age .................................... 1028

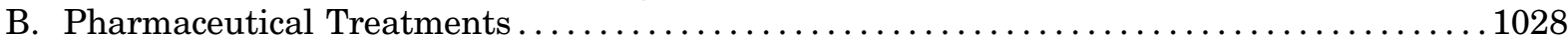

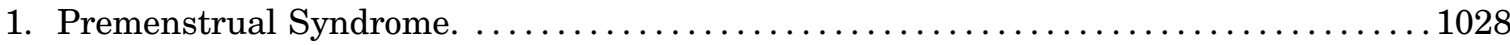

2. Urinary Tract Infections. . . . . . . . . . . . . . . . . . . . . . . . . . . . . . . . . . 1029

3. Pregnancy. .................................................... 1029

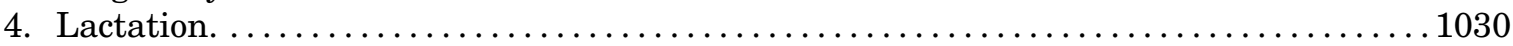

5. Menopausal Symptoms........................................... 1030

C. Botanicals as Alternatives for Women's Health . . . . . . . . . . . . . . . . . . . . . . . . . . . 1030

D. Current Approaches and Techniques in Botanical Research . . . . . . . . . . . . . . . . . . 1031

1. Identification and Procurement of Plant Material. . . . . . . . . . . . . . . . . . . . . . 1031

2. Identification of Bioactive Compounds................................. 1032

3. The Selection and Implementation of Appropriate Screening Bioassays............. 1033

4. Interactions between Phytochemicals in Botanical Extracts. .................... 1034

5. In Vivo Studies; Pharmacokinetic Profile. ................................. 1035

II. Botanicals Used by Premenopausal Women .................................. 1035

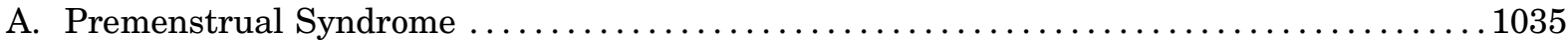

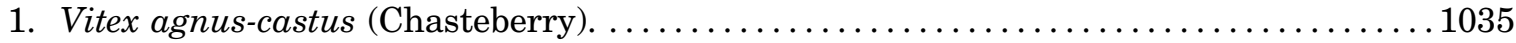

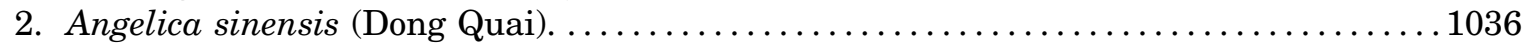

3. Viburnum opulus and Viburnum prunifolium (Cramp Bark and Black Haw). ......1036

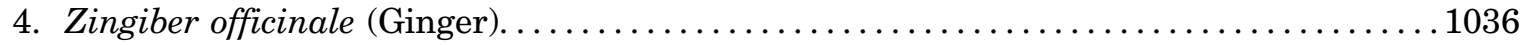

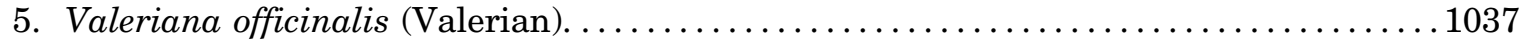

6. Oenothera biennis (Evening Primrose)............................... 1037

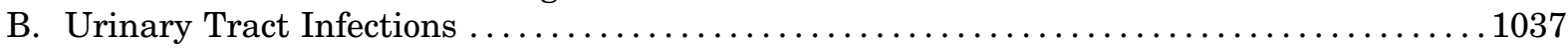

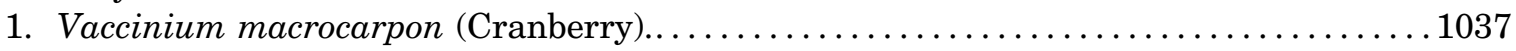

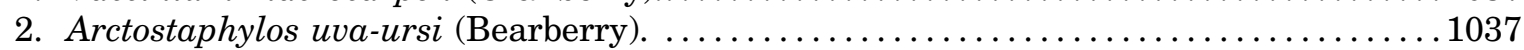

III. Botanicals Used during Pregnancy/Lactation $\ldots \ldots \ldots \ldots \ldots \ldots \ldots \ldots \ldots \ldots \ldots \ldots \ldots \ldots \ldots \ldots$

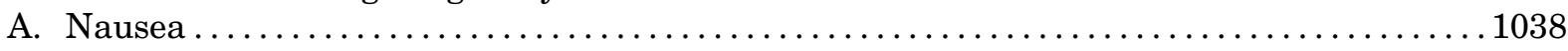

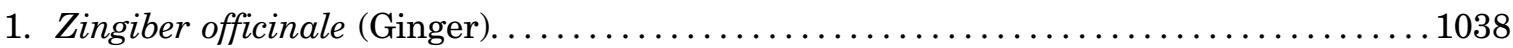

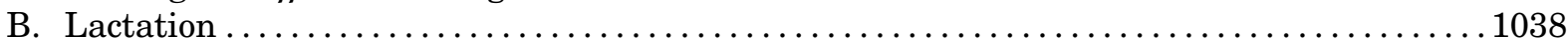

1. Trigonella foenum-graecum (Fenugreek) ............................... 1038

2. Silybum marianum (Milk Thistle)...................................... 1039

IV. Botanicals Used by Menopausal Women. . . . . . . . . . . . . . . . . . . . . . . . . . . . 1040

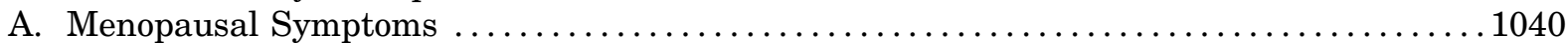

1. Serotonergic Mechanism. .......................................... 1040

a. Actaea /Cimicifuga racemosa (black cohosh). ........................... 1040

b. Valeriana officinalis (valerian).................................... 1040

This work was supported in part by the National Institutes of Health Office of Dietary Supplements (ODS) and National Center for Complementary and Integrated Health (NCCIH) [Grants P50 AT000155 and T32 AT007533].

Address correspondence to: Dr. Judy Bolton, Department of Medicinal Chemistry and Pharmacognosy, College of Pharmacy, University of Illinois at Chicago, 833 South Wood St., M/C 781, Chicago, IL 60612-7231. E-mail: judy.bolton@uic.edu

dx.doi.org/10.1124/pr.115.010843. 
2. Estrogenic Mechanism. ........................................... 1041

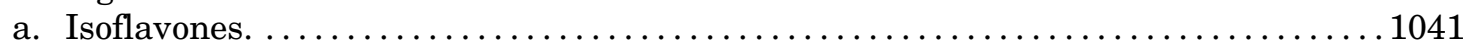

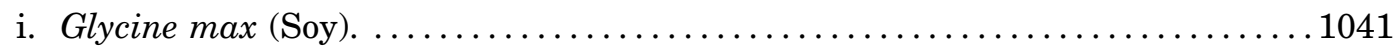

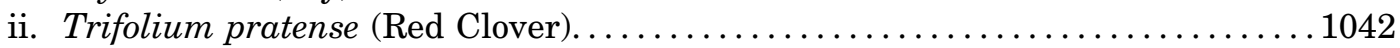

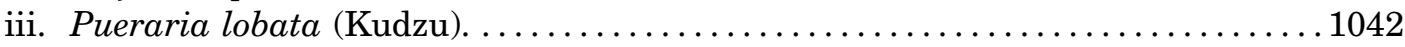

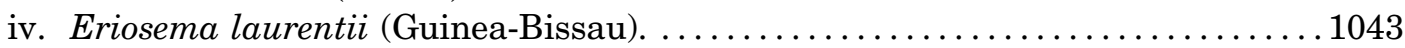

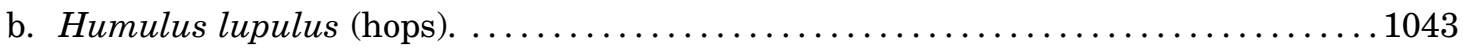

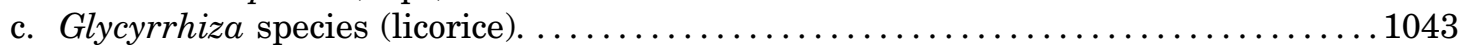

d. Rheum rhaponticum (rhubarb)....................................... 1045

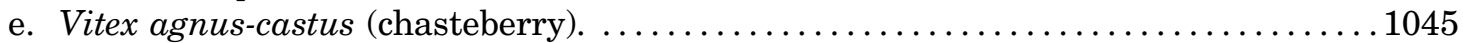

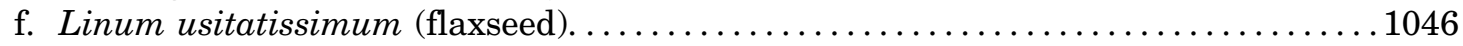

g. Epimedium species (horny goat weed). .............................. 1046

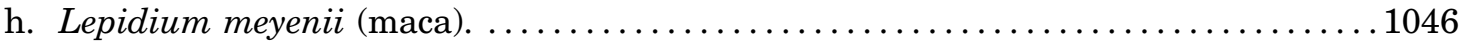

i. Oenothera biennis (evening primrose) . .............................. 1047

j. Medicago sativa (alfalfa)........................................ 1047

3. Unknown Mechanism............................................ 1047

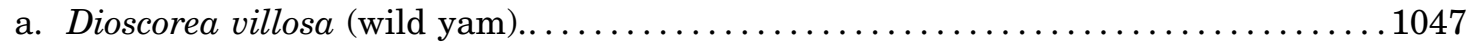

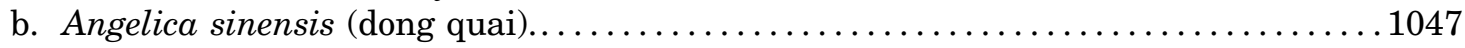

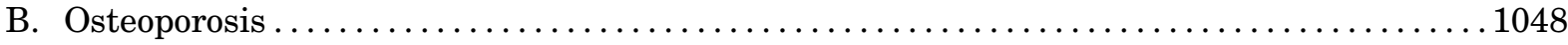

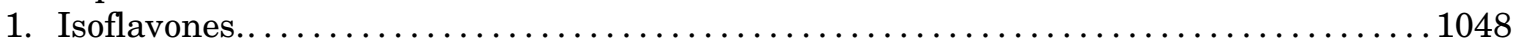

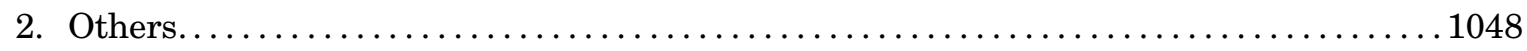

V. Botanicals for Breast Cancer Prevention ...................................... 1048

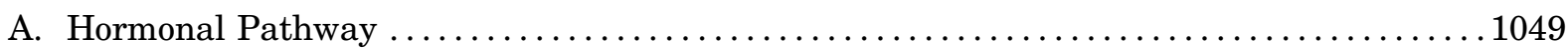

B. Chemical Pathway ................................................. 1049

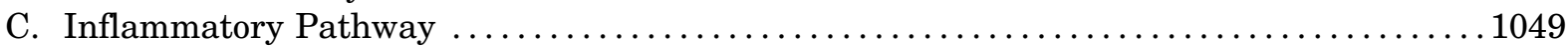

D. Epigenetic Pathway.................................................. 1049

E. Isoflavone-containing Botanicals (Red Clover, Soy) ............................ 1049

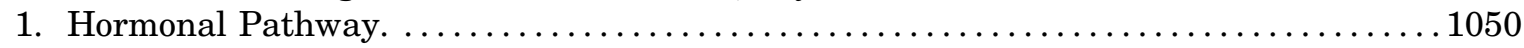

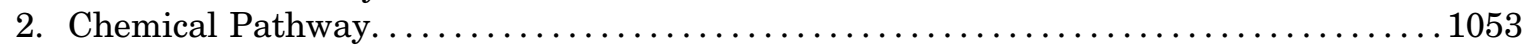

3. Inflammatory Pathway............................................. 1054

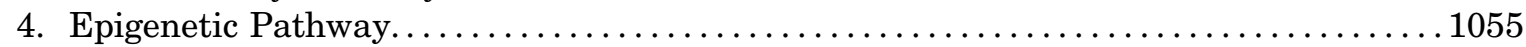

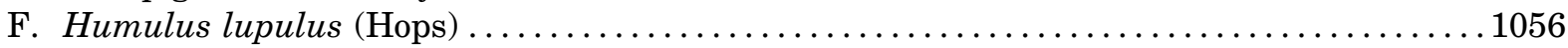

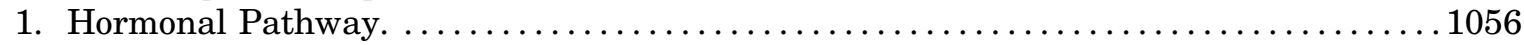

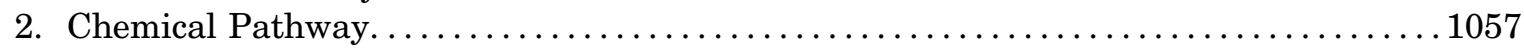

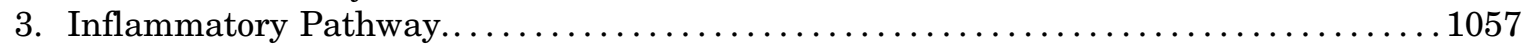

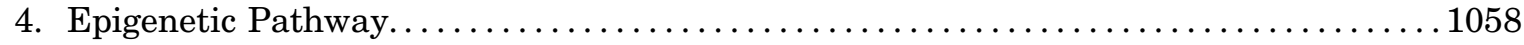

G. Glycyrrhiza Species (Licorice) ....................................... 1058

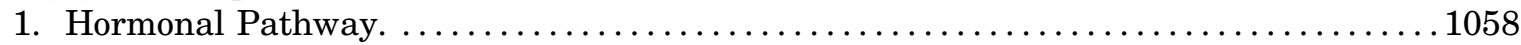

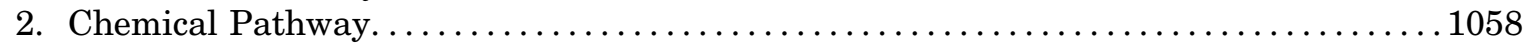

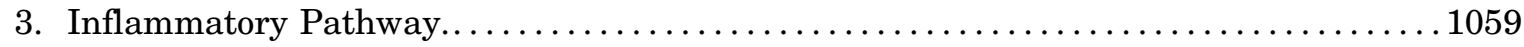

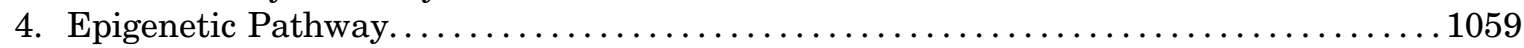

H. Silybum marianum (Milk Thistle) .................................... 1059

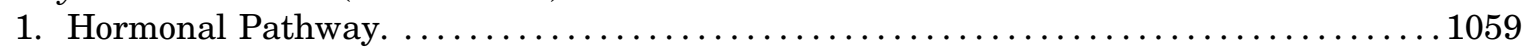

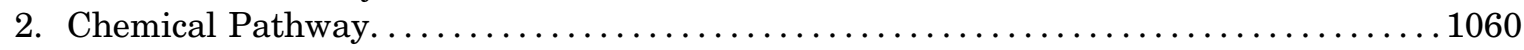

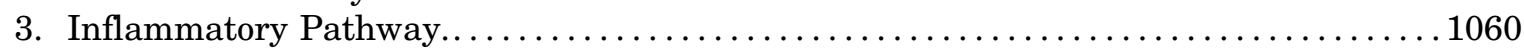

4. Epigenetic Pathway.............................................. 1060

ABBREVIATIONS: AhR, arylhydrocarbon receptor; ARE, antioxidant response element; BaP, benzo(a)pyrene; BGF, bioactivity guided fractionation; BW, body weight; CNS, central nervous system; COX-2, cyclooxygenase 2; DMBA, 7,12-dimethylbenz[a]anthracene; DNMT, DNA methyltransferase; $\mathrm{E}_{2}, 17 \beta$-estradiol; ER, estrogen receptor; ERE, estrogen response element; EROD, ethoxyresorufin-O-deethylase; G. glabra, Glycyrrhiza glabra; G. inflata, Glycyrrhiza inflata; G. uralensis, Glycyrrhiza uralensis; HER2, human epidermal growth factor 2; HT, hormone therapy; 5-HT, 5-hydroxytryptamine (serotonin); $\mathrm{IC}_{50}$, concentration associated with $50 \%$ inhibition of the maximal activity; IKK $\alpha / \beta$, inhibitor of nuclear factor kappa-B kinase subunit alpha/beta; IL, interleukin; iNOS, inducible nitric oxide synthase; IX, isoxanthohumol; Keap1, Kelch-like ECH-associated protein 1; LC-MS/MS, liquid chromatography coupled with tandem mass spectrometry; LPS, lipopolysaccharide; MS, mass spectrometry; NF- $\kappa$ B, nuclear factor kappa-light-chain-enhancer of activated B cells; NQO1, NAD(P)H, quinone oxidoreductase 1; Nrf2, nuclear factor (erythroid-derived 2)-like 2; NSAID, nonsteroidal anti-inflammatory drug; NMR, nuclear magnetic resonance; PGE2, prostaglandin E2; PMS, premenstrual syndrome; 6-PN, 6-prenylnaringenin; 8-PN, 8-prenylnaringenin; SSRI, selective serotonin reuptake inhibitor; TCDD, 2,3,7,8-tetrachlorodibenzo- $p$-dioxin; TPA, 12-O-tetradecanoylphorbol-13-acetate; UTI, urinary tract infection; XH, xanthohumol; XRE, xenobiotic response element. 
I. Angelica sinensis (Dong Quai) ..................................... 1060

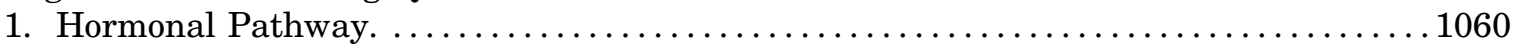

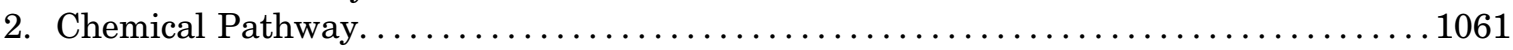

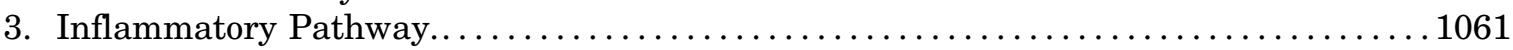

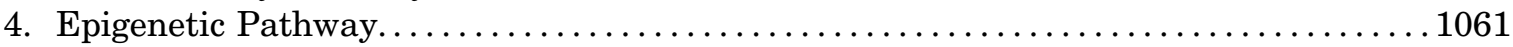

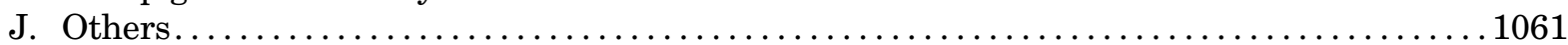

VI. Botanicals/Phytochemicals with Potential Toxic Effects .......................... 1062

VII. Conclusions and Future Directions ................................................ 1064

Acknowledgments .................................................... 1064

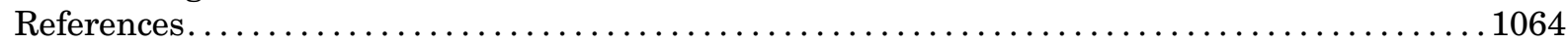

\begin{abstract}
Botanical dietary supplements are increasingly popular for women's health, particularly for older women. The specific botanicals women take vary as a function of age. Younger women will use botanicals for urinary tract infections, especially Vaccinium macrocarpon (cranberry), where there is evidence for efficacy. Botanical dietary supplements for premenstrual syndrome (PMS) are less commonly used, and rigorous clinical trials have not been done. Some examples include Vitex agnus-castus (chasteberry), Angelica sinensis (dong quai), Viburnum opulus/prunifolium (cramp bark and black haw), and Zingiber officinale (ginger). Pregnant women have also used ginger for relief from nausea. Natural galactagogues for lactating women include Trigonella foenum-graecum (fenugreek) and Silybum marianum (milk thistle); however, rigorous safety and efficacy studies are lacking. Older women suffering menopausal symptoms are increasingly likely to use botanicals, especially since the Women's Health Initiative showed an increased risk for breast cancer associated with traditional hormone therapy. Serotonergic mechanisms similar to antidepressants have been proposed for
\end{abstract}

\section{Introduction}

\section{A. Women's Health as a Function of Age}

Women's health and wellbeing are profoundly affected by hormone levels, which can vary dramatically with age, as shown for estrogen in Fig. 1. In addition, older women are more susceptible to illnesses such as cancer, cardiotoxicity, and neurodegenerative diseases (Abouzahr, 2014; de la Cruz et al., 2014; Alzheimer's Association, 2014). These illnesses are stimulated by inflammation as well as exogenous and endogenous stressors (Clark and Vissel, 2013; Wright et al., 2013; Djaldetti and Bessler, 2014; Ren and Xu, 2015). Hormone balance is crucial to stabilizing women's physiology, because hormones control vital biological functions including cardiovascular, respiratory, digestive, reproductive, cerebral, and immune systems. The two main sex hormones in women are estrogen and progesterone (Fig. 2). Estrogen is synthesized in the ovaries and adipose tissue and is responsible for secondary sex characteristics and cell growth. Progesterone balances estrogen's proliferative effect. Hormonal imbalance can lead to several health issues such as obesity, cardiovascular disease, autoimmune disease, breast cancer, and osteoporosis. It is now recognized that women's healthcare must
Actaea/Cimicifuga racemosa (black cohosh) and Valeriana officinalis (valerian). Plant extracts with estrogenic activities for menopausal symptom relief include Glycine max (soy), Trifolium pratense (red clover), Pueraria lobata (kudzu), Humulus lupulus (hops), Glycyrrhiza species (licorice), Rheum rhaponticum (rhubarb), Vitex agnus-castus (chasteberry), Linum usitatissimum (flaxseed), Epimedium species (herba Epimedii, horny goat weed), and Medicago sativa (alfalfa). Some of the estrogenic botanicals have also been shown to have protective effects against osteoporosis. Several of these botanicals could have additional breast cancer preventive effects linked to hormonal, chemical, inflammatory, and/or epigenetic pathways. Finally, although botanicals are perceived as natural safe remedies, it is important for women and their healthcare providers to realize that they have not been rigorously tested for potential toxic effects and/or drug/botanical interactions. Understanding the mechanism of action of these supplements used for women's health will ultimately lead to standardized botanical products with higher efficacy, safety, and chemopreventive properties.

take aging and hormone balance into consideration for quality of life and prevention of disease (Morris and Currie, 2013). However, many women have lost faith in conventional pharmaceuticals because of confusion in recommendations as well as distrust in the medical system that seems to view natural aging and menopause in particular as diseases (Taylor, 2015). This review will focus on women's health botanicals as natural alternatives to traditional pharmaceutical therapies used by premenopausal, menopausal, and postmenopausal women.

\section{B. Pharmaceutical Treatments}

1. Premenstrual Syndrome. A wide range of pharmacological approaches are available to treat premenstrual syndrome (PMS), including oral contraceptives (birth control pills), nonsteroidal anti-inflammatory drugs (NSAIDs), dopamine agonists, diuretic agents, and selective serotonin reuptake inhibitors (SSRIs) (Kelderhouse and Taylor, 2013; Imai et al., 2015) (Fig. $3)$. Birth control pills are effective remedies for premenopausal women to relieve menstrual cramping as well as preventing pregnancy. They suppress ovulation and thin the endometrial lining, which reduces menstrual fluid volume along with prostaglandin levels, which in turn decreases the pain associated with uterine 


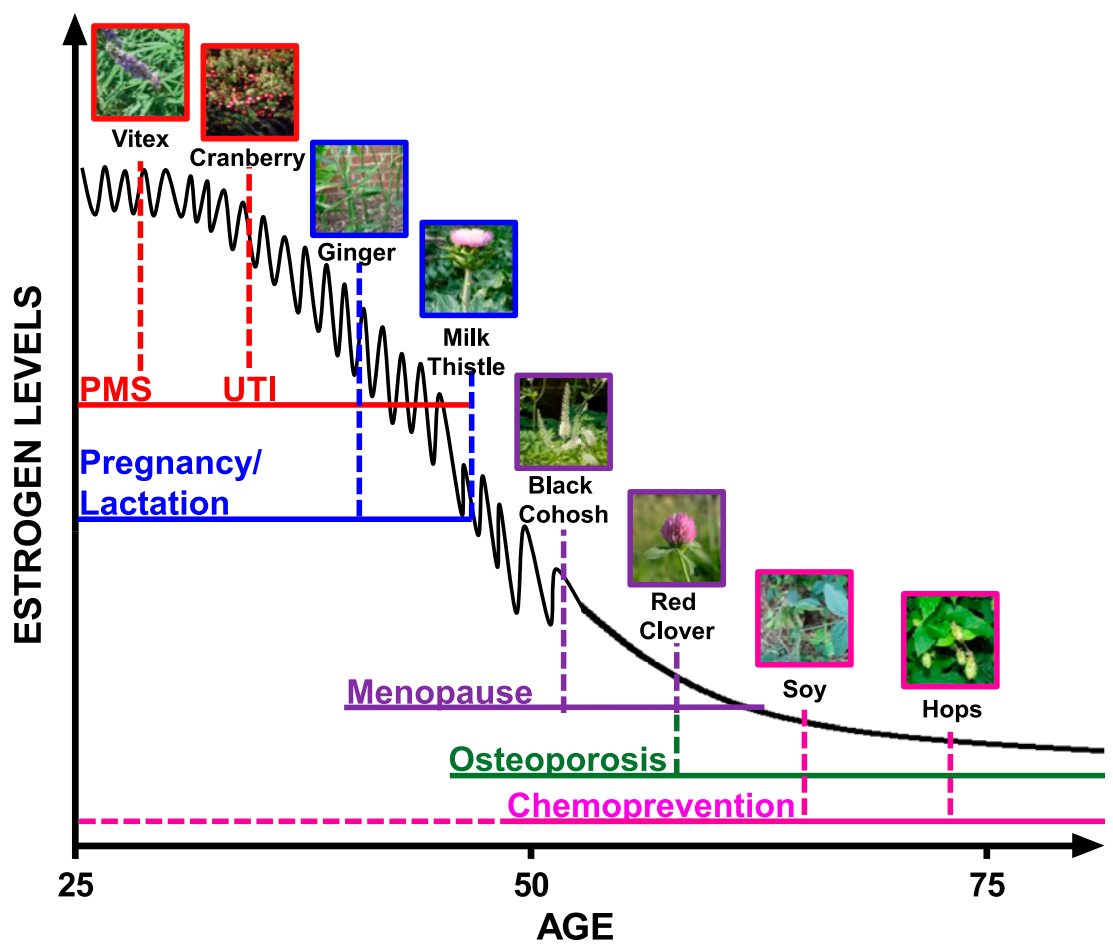

Fig. 1. Botanicals women take as a function of age. The popularity of botanical dietary supplements varies as a function of age, hormone levels, and usage.

contractions (Creatsas et al., 1990; Zahradnik et al., 2010). Side effects include nausea, water retention, and weight gain and birth control pills may not be suitable for some women, especially those wanting to become pregnant. NSAIDs such as ibuprofen (Fig. 3) reduce myometrial activity by inhibiting prostaglandin synthesis and reducing vasopressin secretion; however, the failure rate is often $25 \%$ and many women cannot tolerate NSAIDs due to side effects that can include bleeding, ulcers, vomiting, and diarrhea (Zahradnik et al., 2010). SSRIs have demonstrated efficacy for treating PMS in randomized placebo-controlled clinical trials (Marjoribanks et al., 2013); however, significant side effects have been reported particularly in the beginning of treatment including headache, nausea, insomnia, fatigue, diarrhea, dizziness, sexual side effects, and reduced concentration (Freeman, 2010).

2. Urinary Tract Infections. Most uncomplicated urinary tract infections are caused by bacteria that enter the urethra and then the bladder where the infection develops (Little et al., 2010). Women tend to have more frequent urinary tract infections (UTIs),
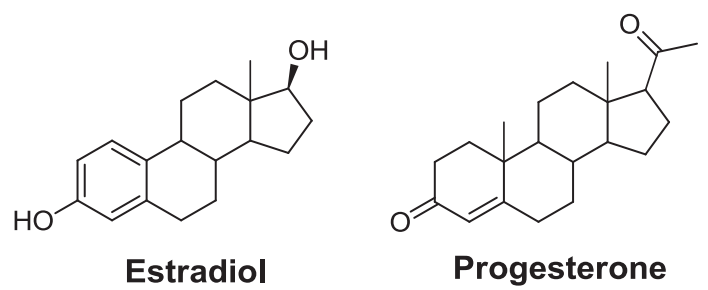

Fig. 2. Structures of estradiol and progesterone. because their urethra is shorter and closer to the anus than in men. UTIs are usually treated with sulfa drugs and antibiotics (Fig. 4). The major problem with excessive antibiotic use, particularly to treat recurring UTIs, is development of drug-resistant bacteria (Nickel, 2007).

3. Pregnancy. The most common adverse symptom associated with pregnancy is morning sickness. Over the years there have been several reports of teratogenic adverse drug reactions associated with pregnancy medications (Saunders and Saunders, 1990). The most sensational of these was the stunted limb growth and severe birth abnormalities associated with exposure to thalidomide (Fig. 5). Similar severe birth defects appeared with the development of secondary sex characteristics in the children of women exposed in utero to diethylstilbestrol (Fig. 5). These sobering examples help explain why women are very reluctant to take prescription drugs for morning sickness. Some recent data may indicate that drugs such as metoclopramide (Fig. 5) may be safe and offer some relief to women when morning sickness severely impacts their quality of life (Lee and Saha, 2011). For example, a recent Danish study on 40,000 women taking metoclopramide (reglan) during pregnancy showed no statistically significant increase in birth defects or miscarriages (Pasternak et al., 2013). However, likely because of the tragic history of drugs prescribed for morning sickness as well as the recommendation by most doctors to avoid prescription drugs entirely during pregnancy, women often look for natural alternatives to control symptoms. 
A Birth control pills<smiles>C#CC1(O)CCC2C3CCC4=CC(=O)CCC4C3CCC21C</smiles>

Norethindrone<smiles>C#CC1(O)CCC2C3CCc4cc(O)ccc4C3CCC21C</smiles>

Ethinyl estradiol
B NSAIDs<smiles>CC(C)Cc1ccc(C(C)C(=O)O)cc1</smiles>

Ibuprofen
C Dopamine agonist<smiles>CC(C)CC1C(=O)N2CCCC2C2(O)OC(C(=O)NC3C=C4c5cccc6[nH]c(Br)c(c56)CC4N(C)C3)(C(C)C)C(=O)N12</smiles>

Bromocriptine
D Diuretic agent

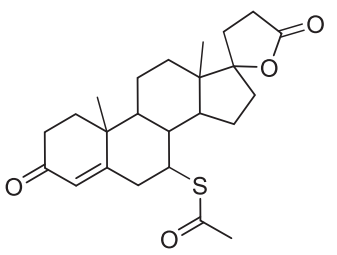

Spironolactone
E SSRI<smiles>CCOC(CCNC)C(CCOc1ccc(C(F)(F)F)cc1)c1ccccc1</smiles>

Fluoxetine

Fig. 3. Examples of prescription drugs used to treat premenstrual syndrome.

4. Lactation. The most frequent cause of breastfeeding failure is decreased milk production. There are a number of prescription drugs that stimulate lactation including metoclopramide (reglan, Fig. 5), domperidone (motilium, motilidone, Fig. 6), and sulpiride (dolmatil, sulparex, Fig. 6) (Zuppa et al., 2010; Rowe et al., 2013). For example, a modest increase in breast milk volume $(100 \mathrm{ml} /$ day $)$ has been reported in mothers of preterm infants given domperidone (Donovan and Buchanan, 2012). However, side effects have been reported with prescription galactagogues, including severe depression, nausea, gastric upset, and diarrhea, and along with the fear that these drugs might contaminate breast milk, many women prefer natural galactagogues, which are perceived as safe (Gabay, 2002; Marcellin and Chantry, 2015).

5. Menopausal Symptoms. Hot flashes and night sweats are the most commonly experienced menopausal symptoms contributing to adverse quality of life (Nelson, 2008). The gold standard of care for menopausal symptoms before the release of the Women's Health Initiative was hormone therapy (HT). Oral conjugated equine estrogens [i.e., estradiol (Fig. 2), equilin (Fig. 7), equilenin] were the most commonly used estrogen formulations
A Antibiotic<smiles>O=C(O)c1cn(C2CC2)c2cc(N3CCNCC3)c(F)cc2c1=O</smiles>

Ciprofloxacin
B Sulfa drug

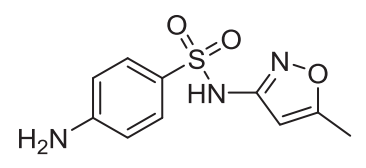

Sulfamethoxazole
Fig. 4. Examples of prescription drugs used to treat UTIs. either alone or in combination with a progestin (Fig. 7) (Bhavnani and Stanczyk, 2014; Stanczyk and Bhavnani, 2014). Since the release of the Women's Health Initiative report in 2002 (Rossouw et al., 2002) and the Million Women study in 2003 (Beral and Million Women Study Collaborators, 2003), women have been discouraged from using traditional HT because of the increased risk of breast cancer, coronary heart disease, stroke, and pulmonary embolism within the estrogen plus progestin arm and increased risk of stroke within the estrogen arm (Brass, 2004; Chlebowski et al., 2013; Manson and Branch, 2014). Despite recent analysis that suggests that women benefit from early initiation of HT with no significant increased breast cancer risk, women still associate HT with these detrimental side effects (Schierbeck et al., 2012; Lacey, 2014). Nonhormonal therapies are also prescribed such as antidepressants, which may act through serotonergic mechanisms. The data suggest that the SSRIs, serotonin-norepinephrine reuptake inhibitors, have efficacy; however, the relief from hot flashes and night sweats are much less than observed with traditional HT and side effects and cost may restrict use for most women (Nelson et al., 2006; Shen and Stearns, 2009).

\section{Botanicals as Alternatives for Women's Health}

Largely as the result of adverse effects reported for prescription medications for women's health (i.e., Women's Health Initiative and increased breast cancer risk), women are frequently turning to botanicals because they are perceived as safe despite the lack of supporting rigorous scientific documentation on either safety or efficacy (North American Menopause Society, 2004, 2011; Lloyd and Hornsby, 2009; Hajirahimkhan et al., 2013a; GarciaCazarin et al., 2014; Lindstrom et al., 2014; van Breemen, 


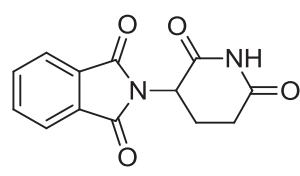

Thalidomide

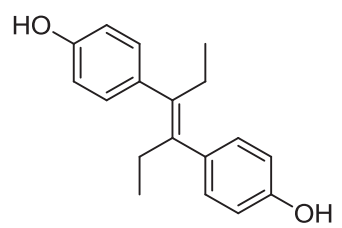

Diethylstilbestrol<smiles>CCN(CC)CCNC(=O)c1cc(Cl)c(N)cc1OC</smiles>

Metoclopramide

Fig. 5. Examples of prescription drugs used to treat morning sickness or possible miscarriages.

2015). The sales of herbal dietary supplements reached 6.4 billion in 2014, with women as the major users taking these botanicals for a variety of ailments (Blumenthal et al., 2015). The rank order in sales of women's health botanicals presented in Table 1 and Fig. 1 shows the popularity of botanicals as a function of a woman's age. However, scientific data about the efficacy of botanicals in women's health and their role in preventing diseases is scarce, and regulations for biological and chemical standardization of these botanicals before marketing is lacking (Snelten et al., 2012; Dietz and Bolton, 2013; Hajirahimkhan et al., 2013a). One of the major problems with clinical research on botanical dietary supplements is the large placebo effect, which is often observed particularly with botanicals which affect the CNS (Nedrow et al., 2006; Geller et al., 2009; Wong et al., 2009). These placebo effects often lead to equivocal and nonsignificant effects, making recommendations on efficacy difficult. In fact, the placebo effect could explain the increase in use of botanical dietary supplements especially as the population ages. Older women are also more likely to use botanicals because they have increased health needs and probably more disposable income.

\section{Current Approaches and Techniques in Botanical Research}

Current research in mechanism of action of botanicals involves a multifaceted approach including phytochemical, biological, and analytical techniques (van Breemen, 2015) (Fig. 8). Numerous challenges are encountered including the 1) identification and procurement of reproducible plant material, 2) the identification of bioactive chemical compounds, 3 ) the selection and implementation of appropriate screening bioassays, 4) potential interactions between the phytoconstituents in a botanical extract, and 5) the analysis of in vivo effects including their metabolism and distribution profile.

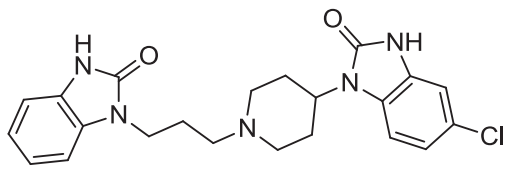

Domperidone

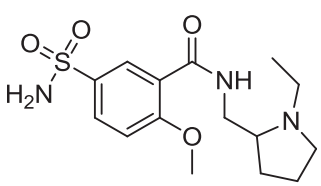

Sulpiride
Fig. 6. Examples of prescription drugs used to increase milk production (galactagogues).
1. Identification and Procurement of Plant Material. Commercial botanicals used for women's health have a long history of traditional use with little scientific evidence of efficacy, safety, or other potential health benefits. For robust scientific studies on these botanicals it is crucial to have a reliable source for the procurement of plant material, because many factors such as plant location, temperature, harvest time, as well as postharvest processing steps, such as drying, can have significant impact on the phytochemical profile of the plant material (Pferschy-Wenzig and Bauer, 2015). In addition, correct authentication of the plant material is essential. Misidentifications can occur due to name similarities. For example, in a dietary supplement formulation the roots of Stephania tetrandra were inadvertently substituted with the roots of Aristolochia fangchi because of the close similarity of the Chinese names leading to an endemic nephropathy including end-stage kidney failure (Vanherweghem, 1998; Dietz and Bolton, 2007). Moreover, misidentification can occur, when the pharmacologically used plant parts, for example roots, of similar species are undistinguishable or if the plant part is provided as powdered material (Dunlap et al., 2015; Simmler et al., 2015a). A visual differentiation of the plant species is usually possible when the whole plant is examined; however, other pharmacognostic methods become necessary for the individual plant part (Simmler et al., 2015b). The botanical integrity may be analyzed by microscopy using taxonomic keys. In many cases, this might not be sufficient to unequivocally identify the raw plant material or powder and in these cases DNA barcoding might be required. Licorice is an example in which DNA barcoding became necessary to unequivocally identify the different species. The roots of the three

A Equine estrogen

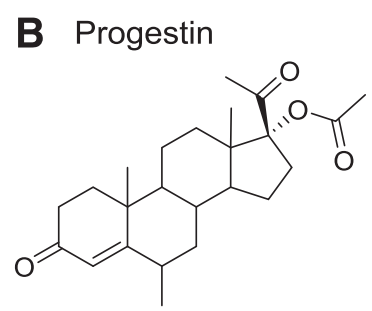

Equilin

Medroxyprogesterone acetate

Fig. 7. Examples of prescription drugs used for menopausal symptoms. 
TABLE 1

Popular Women's Health Dietary Supplements (Rank Order Popularity 2014)

From Blumenthal et al., 2015.

\begin{tabular}{|c|c|c|c|c|c|}
\hline Rank & Latin Name & Common Name & Use & Reported Mechanism & Proposed Phytochemical \\
\hline 2. & Vaccinium macrocarpon & Cranberry & UTI & Bacterial adhesion & Proanthocyanidins \\
\hline 4. & Actaea / Cimicifuga racemosa & Black cohosh & Menopause & Serotonin & $N$-methylserotonin \\
\hline 5. & Linum usitatissimum & Flaxseed & Menopause $^{a}$ & Estrogen & Enterodiol/lenterolactone \\
\hline 6. & Valeriana officinalis & Valerian & Menopause/PMS ${ }^{b}$ & Serotonin & Valerenic acid \\
\hline 10. & Zingiber officinale & Ginger & Nausea/PMS ${ }^{c}$ & Prokinetic & Gingerols/shogaols \\
\hline 12. & Silybum marianum & Milk thistle & Lactation $^{d}$ & Prolactin/estrogen & Silybin B \\
\hline 16. & Epimedium species & Horny goat weed & Menopause/PMS & Estrogen & Icaritin $->$ desmethylicaritin \\
\hline 27. & Lepidium meyenii & Maca & Menopause & Estrogen & Phytosterols \\
\hline 28. & Trigonella foenum-graecum & Fenugreek & Lactation & Estrogen & Diosgenin, apigenin, luteolin \\
\hline \multirow[t]{5}{*}{29.} & Isoflavones & & & & \\
\hline & Glycine $\max$ & Soy & Menopause/bone & Estrogen & Genistein, daidzein -> equol \\
\hline & Trifolium pratense & Red clover & Menopause/bone & Estrogen & Biochanin A $->$ genistein \\
\hline & Pueraria lobata & Kudzu & Menopause & Estrogen & Puerarin $->$ daidzein \\
\hline & Eriosema laurentii & Guinea-bissau & Menopause & Estrogen & Lupinalbin A \\
\hline 37. & Oenothera biennis & Evening primrose & Menopause/PMS ${ }^{e}$ & Estrogen & $\gamma$-linolenic acid \\
\hline \multicolumn{6}{|c|}{ Not ranked (out of top 40)f } \\
\hline & Angelica sinensis & Dong quai & Menopause/PMS & SERM/unknown & Ligustilide \\
\hline & Arctostaphylos uva-ursi & Bearberry & UTI & Antibacterial & Arbutin \\
\hline & Dioscorea villosa & Wild yam & Menopause/PMS & Estrogen & Diosgenin \\
\hline & Glycyrrhiza species & Licorice & Menopause & Estrogen & Liquiritigenin \\
\hline & Humulus lupulus & Hops & Menopause $^{b}$ & Estrogen & Xanthohumol -> 8-PN \\
\hline & Medicago sativa & Alfalfa & Menopause & Estrogen & Coumestrol \\
\hline & Rheum rhaponticum & Rhubarb & Menopause & Estrogen & Piceatannol \\
\hline & Vitex agnus-castus & Chasteberry & Menopause/PMS & CNS/estrogen & Apigenin/penduletin \\
\hline & Viburnum opulus/prunifolium & Cramp bark/black haw & PMS & Antispasmodic & Scopoletin \\
\hline
\end{tabular}

SERM, selective estrogen receptor modulator.

${ }^{a}$ More commonly used for lipid lowering and anti-inflammatory.

${ }^{b}$ More commonly used as a sedative.

${ }^{c}$ More commonly used for anti-inflammatory and immune modulation.

${ }^{d}$ More commonly used for liver detoxification.

${ }^{e}$ More commonly used to treat eczema.

${ }^{f}$ Alphabetical order.

medicinally used licorice species, Glycyrrhiza uralensis, G. glabra, and G. inflata are very similar and difficult to distinguish although they are quite different chemically and biologically (Kondo et al., 2007b). The use of DNA barcoding made it possible to differentiate these three species and to even distinguish hybrids of these plants (Simmler et al., 2015a). After the preparation of botanical extracts, an array of analytical methods are available to perform phytochemical analyses [e.g., thin-layer chromatography, liquid chromatography coupled with tandem mass spectrometry, LC-MS/MS and nuclear magnetic resonance (NMR)] to unambiguously identify the plant material used for the preparation of the extract (Simmler et al., 2015b).

2. Identification of Bioactive Compounds. To obtain efficacious and safe plant based remedies, it is essential to identify the bioactive and toxic phytoconstituents as well as to describe their mechanism of action (van Breemen, 2015). For some herbal dietary supplements the major bioactive constituents for the indicated medical use are known. For example the observed in vitro estrogenic activity of soy and red clover extracts are mainly due to the isoflavones, genistein and biochanin A (Overk et al., 2005; Hajirahimkhan et al., 2013a) (Fig. 24). However, other botanicals have been medicinally used for years

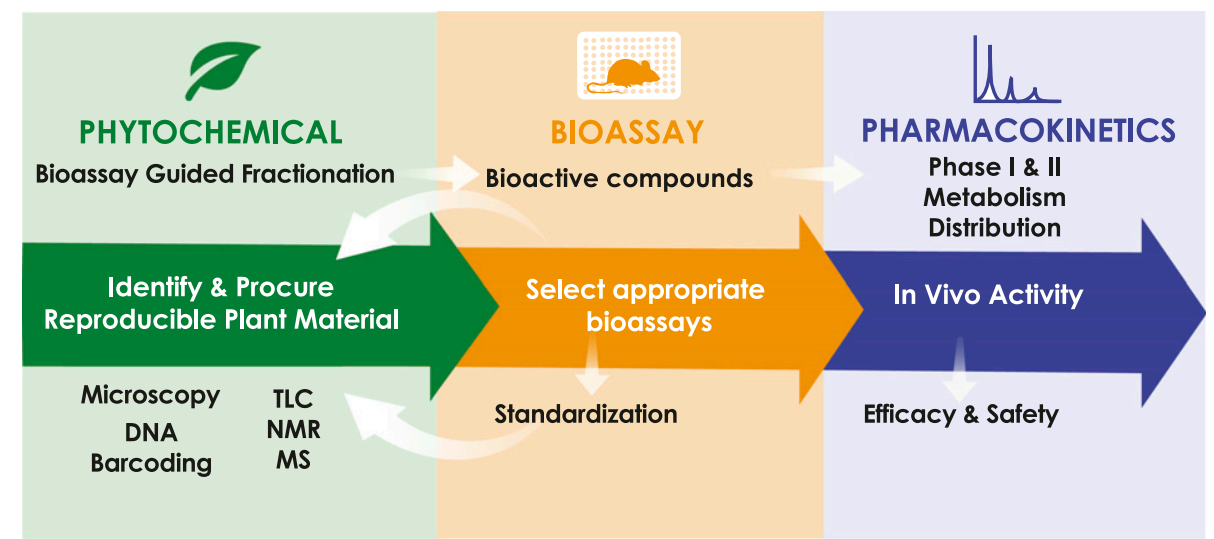

Fig. 8. Integration of phytochemistry, bioassay, and pharmacokinetics to standardize botanicals to bioactive phytochemicals. 
without a clear knowledge of the bioactive constituents (Qiu et al., 2014). Extracts of the roots of black cohosh (Actaea/Cimicifuga racemosa) are an example of a plant that has been standardized to a lead compound, the triterpene, 23-epi-26-deoxyactein (formerly 27-deoxyactein) (Fig. 9) (Chen et al., 2002); however, there is no evidence that the triterpenes have any effect on menopausal symptom relief (Qiu et al., 2014). The active compounds in black cohosh have not been clearly identified, although, as discussed in section IV.A.1, $N_{\omega}$-methylserotonin has been isolated as a potential serotonin receptor agonist (Fig. 9) (Powell et al., 2008). In preclinical and clinical investigations of botanicals, it is desirable to use extracts that have been chemically and biologically standardized to the active compounds to analyze a safe and efficacious dose for these botanical products. In addition, once the bioactive compounds are known optimal extraction procedures (choice of extraction solvent, defatting) can be used to enrich the active compounds. The comparative analysis of many clinical studies with botanical extracts is often problematic, because various extracts with different solvents are used and therefore comparisons or conclusions are difficult to draw (Qiu et al., 2014; van Breemen, 2015).

When pharmacological/clinical studies are done with individual phytoconstituents, the purity and appropriate analysis methods should be reported (Betz et al., 2011; Pauli et al., 2014). This is especially important when easily degradable compounds are analyzed. This has been a particular problem with studies on Angelica sinensis, because the proposed bioactive compound Z-ligustilide is particularly unstable (Dietz et al., 2008) (Fig. 10). In these cases quality control analysis of these compounds may have to be performed at regular intervals throughout the studies (Schinkovitz et al., 2008). In this review, we put higher emphasis on studies that used well-analyzed bioactive compounds and standardized extracts for pharmacological and clinical investigations.

3. The Selection and Implementation of Appropriate Screening Bioassays. Because of the complex nature of botanical extracts with a multitude of phytoconstituents, robust and medium to high-throughput assays
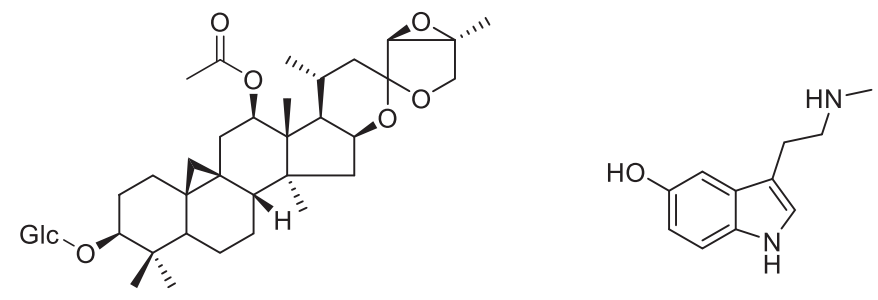

23-epi-26-deoxyactein

(marker compound)

No-methylserotonin (serotonergic)

Fig. 9. Phytochemicals in black cohosh.

that tolerate crude extracts or fractions/partitions are desirable for the characterization of bioactive and toxic compounds (Piersen et al., 2004). Various problems might occur during the biological screening process of natural products. These issues were recently reviewed in more detail (Sink et al., 2010; Thorne et al., 2010; Bisson et al., 2016). Briefly, some phytochemicals may give false-positive results because of nonspecific enzyme or protein interactions, formation of aggregates that may bind to protein targets, detergent-like effects, membrane disruption, cytotoxicity in cell based assays, precipitation of an analyte, and interference with detection methods, such as ultraviolet-, luminescence-, or fluorescence detection (Piersen et al., 2004; Bisson et al., 2016). Therefore, modifications of the bioassay, removal of interfering compounds, such as fatty acids, additional controls, use of additional computational methods, inclusion of literature data, and additional bioassays to test for interference or functional activity using different detection systems might be necessary to ensure functional and meaningful biological results (Piersen et al., 2004; Bisson et al., 2016). Ultimately, in vivo tests will be required to analyze the clinical potential of these phytochemicals/ botanical extracts. Often not one single compound is responsible for the activity of the extract, but rather several phytoconstituents that lead to the observed overall activity through interactive processes, e.g., additive, synergistic, or antagonistic effects (Piersen et al., 2004; Gertsch, 2011). In general, to identify bioactive compounds in multicompound extract mixtures a variety of different approaches might be used as outlined below.

i. Bioactivity guided fractionation (BGF) tries to connect analytical techniques with a biological/ biochemical assay, to isolate substances of biological relevance (Weller, 2012; van Breemen, 2015). BGF aims to identify the phytoconstituents that are responsible for a certain bioactivity of the botanical extract (Agarwal et al., 2014). Different chromatographic techniques may be used to separate the extract into fractions, which will be tested in a particular bioassay, and the most active fractions will be further separated (Weller,

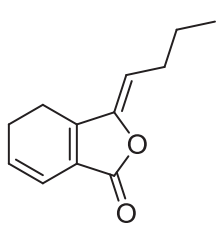

Z-Ligustilide (chemopreventive)

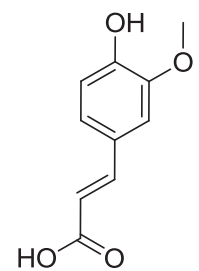

Ferulic acid (anti-inflammatory)
Fig. 10. Phytochemicals in dong quai. 
2012). An example is the fractionation of a black cohosh extract using the $5-\mathrm{HT}_{7}$ receptor binding activity of the black cohosh fractions as the bioassay target (Powell et al., 2008). Subsequently, the potent, 5 - $\mathrm{HT}_{7}$-ligand, $N_{\omega}$-methylserotonin (Fig. 9), was identified in the active black cohosh fractions (Fig. 11). A parallel detection of possible known compounds by LC-MS/MS using databases or NMR and structure elucidation in the active fractions is important to avoid isolation of known compounds (dereplication) (Weller, 2012; Wolfender et al., 2015). The compounds that have been isolated or identified through BGF can also be tested in several other assays including functional cell based assays that generally show less interference with fatty acids or chlorophyll leading to false-positive results. Also, using human cell culture models has the advantage that they might better predict human-compound interactions before preclinical and clinical trials (Nierode et al., 2016). However, often BGF of a botanical extract does not lead to one strongly active compound but to several weakly active compounds due to possible interactive/synergistic processes (Piersen et al., 2004). Another reason might be that the isolated, purified compounds might be unstable, such as $Z$-ligustilide from Angelica sinensis discussed above (Fig. 10); however, these compounds may be stabilized in the whole plant matrix (Schinkovitz et al., 2008).

ii. Mass spectrometry (MS) screening might present a higher-throughput alternative than BGF (van Breemen, 2015), because MS-based screening eliminates the laborious process of fractionation by using affinity extraction steps for example (e.g., magnetic bead affinity MS-based screening) (van Breemen, 2015; Wolfender et al., 2015). The bound ligands or compounds that lead to a reaction with the analyzed enzyme can immediately be characterized by MS (van Breemen, 2015; Wolfender et al., 2015). In addition to LC-MS/MS, biological assays can also be coupled with planar chromatographic methods, such as thin layer chromatography (bioautography) (Wolfender et al., 2015). This method has often been used for the identification of antibacterial, antifungal, and antioxidant or enzyme inhibiting compounds in the target-directed isolation of active phytoconstituents of natural products (Dewanjee et al., 2015). The advantage of these online biological identification methods are that they are faster and require less plant material or botanical extracts. However, to verify the activity and for mechanism of action studies it is necessary to have the purified fully characterized compound.

iii. Compound-directed analysis. Many different methods, mainly LC-MS/MS and NMR techniques,

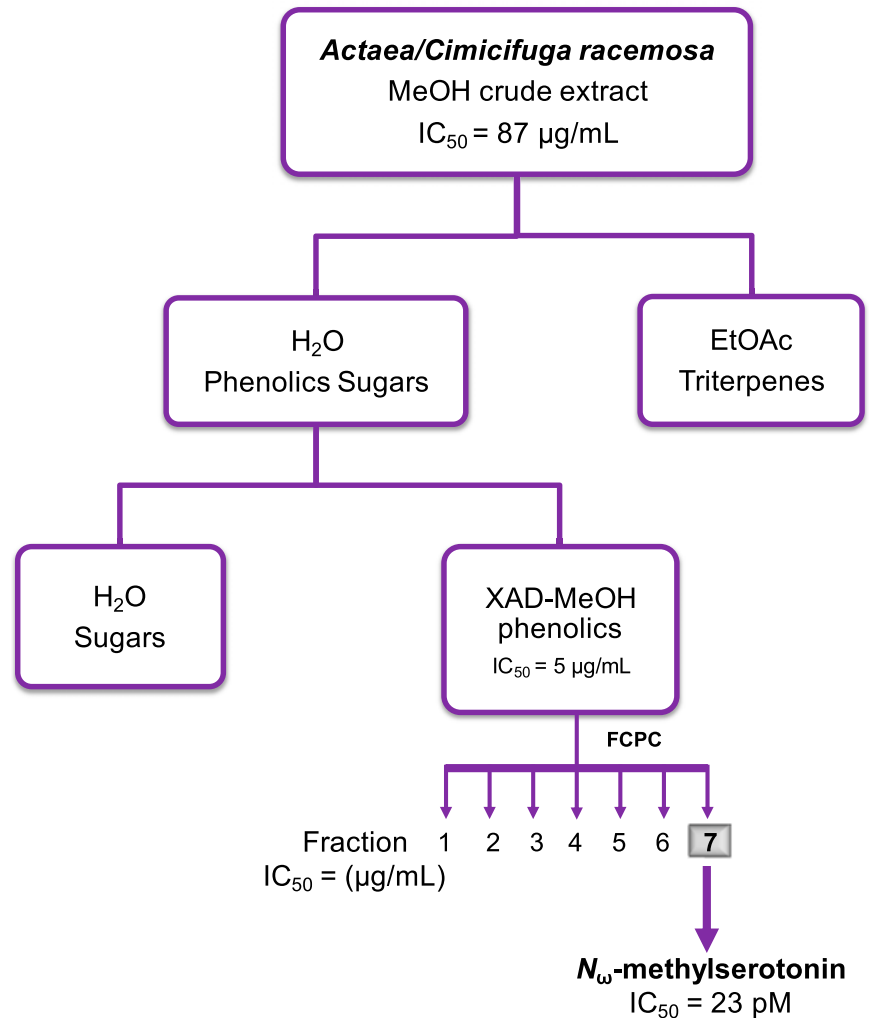

Fig. 11. Example of bioassay guided fractionation using black cohosh modified from Powell et al., 2008.

enable the identification of several phytoconstituents "metabolite profiling" in a complex mixture with subsequent data mining tools (Wolfender et al., 2015). Several metabolomic techniques recently advanced this approach and were reviewed in detail elsewhere (Fillet and Frederich, 2015; Wolfender et al., 2015). This approach puts the emphasis on the identification of phytoconstituents in a plant or microorganism. After dereplication of known compounds, unknown constituents need to be isolated from the plant or other matrices. Subsequently, these compounds can be tested in several bioassays. Unanticipated effects might be discovered by this method. Alternatively, if a targeted biological examination is desired, this might lead to a quicker discovery of novel and active phytochemicals (Wu et al., 2015a).

4. Interactions between Phytochemicals in Botanical Extracts. Because botanical extracts are multicompound mixtures, the different phytoconstituents might influence each other, leading to additive, synergistic, and/or antagonistic effects (Wagner, 2011; Pelkonen et al., 2014). Therefore, the activity of the extracts might not be solely the responsibility of one or two compounds, but a multitude of compounds might be involved. Because of the difficulty in analysis of multiple interactions between the phytoconstituents 
in botanical extracts, these effects have not been well studied and much more research is needed in this field (Gertsch, 2011). Synergistic or antagonistic activities of phytoconstituents can be analyzed, for example by the isobol method or the Chou-Talalay combination index (Chou and Talalay, 1984). By using these methods, synergistic effects of 6-gingerol in a ginger extract (Fig. 15) on inhibition of prostate cancer cell growth and synergistic antimicrobial effects of constituents in Hydrastis canadensis have been demonstrated (Junio et al., 2011; Brahmbhatt et al., 2013). Although some investigations have analyzed synergistic or antagonistic effects of phytochemicals with drugs or other botanicals (Einbond et al., 2006), studies to analyze the interactions of phytochemicals in one botanical used for women's health are mostly lacking. Other interactions between phytochemicals in a plant extract are possible, such as stabilization of one compound by other compounds in an extract and facilitation of absorption and therefore higher bioavailability by compounds in the extract matrix rather than isolated compounds. In general, the complexity of botanical extracts is often seen as an advantage and as a promising strategy to manage complex chronic diseases, such as neurodegenerative disorders through targeting multiple pharmacological targets by a complex extract (Hopkins, 2008; $\mathrm{Li}$ et al., 2011). This is in contrast to the classic paradigm of single target-single drug and requires appropriate analysis of influences on network pharmacology using genomic or proteomic approaches combined with functional assays (Pelkonen et al., 2014).

5. In Vivo Studies; Pharmacokinetic Profile. After bioactive extracts standardized to the phytochemical and potential toxic constituents have been established, in vivo studies have to be performed (van Breemen, 2015). A limiting factor of the clinical bioactivity of phytoconstituents is often the extensive metabolism in vivo. Many active botanical compounds are polyphenols and therefore prone to extensive metabolism. For example, liquiritigenin (Fig. 27) from licorice and curcumin from turmeric form multiple glucuronides (Ganesan et al., 2015; Hajirahimkhan et al., 2015). In the case of curcumin, efforts are underway to improve its bioavailability and therefore enhance its clinical efficacy (Ganesan et al., 2015). The in vivo studies will give essential information about the metabolism profile, pharmacokinetic, and distribution profile. In addition, the animal models have to be selected to test for the desired efficacy and safety. For example, the estrogenic activity of plant extracts can be evaluated with immature Sprague-Dawley rats that do not produce estradiol and with ovariectomized Sprague-Dawley rats (Overk et al., 2007, 2008). These animals are very susceptible to compounds with estrogenic activity, such as phytoestrogens, and would show an increase in uterine weight after a few days of estrogenic treatment. After the in vivo activity of the botanical extracts and their bioactive constituents have been determined and the induction/inhibition profile of human P450 enzymes has been established in animal models, ideally doubleblind, placebo-controlled, randomized human clinical trials should be performed with the standardized extract or well described phytochemical to ensure clinical efficacy and safety (van Breemen, 2015).

To fulfill all these steps necessary for the analysis of pharmacological mechanisms of botanicals and their standardization, a multidisciplinary team of researchers is necessary. An example of a well-established team is the UIC/NIH Center for Botanical Dietary Supplements Research (UIC Botanical Center), which has been investigating the safety and mechanisms of action of botanical dietary supplements consumed by women since 1999 for the purpose of maintaining good health and quality of life, especially during the transition to and through menopause (Farnsworth et al., 2008). This Botanical Center involves a synergistic team of multidisciplinary investigators with complementary expertise who are organized into three Projects (Phytochemistry/standardization, Biological evaluation, Metabolism and safety) and three research Cores (Botanical, Bioassay, Chemical) (Fig. 12). The organization is very similar to the general scheme shown in Fig. 8.

\section{Botanicals Used by Premenopausal Women}

\section{A. Premenstrual Syndrome}

Menstrual cramps are a very common ailment in premenopausal women experienced by approximately $50 \%$ of women starting with first menses (Dawood, 2006). Pain usually starts within hours of menstrual bleeding and peaks during the heaviest flow on the second or third day of the cycle. Cramps can worsen as women age due to hormone imbalance and other conditions associated with this including uterine fibroids and adenomyosis. These cramps are caused by the release of prostaglandins that induce spasms in the uterine muscle. As a result, botanicals with smooth muscle relaxing properties could be effective antispasmodic agents (Park et al., 2014; van Andel et al., 2014). Botanicals traditionally used to treat menstrual disorders are reviewed below.

1. Vitex agnus-castus (Chasteberry). Chasteberry is obtained from the dried ripe fruit of the chaste tree and has been used as a women's health botanical since ancient Greece (Wuttke et al., 2003). One mechanism of action could be through the dopamine receptors, which decrease thyrotropin releasing hormone and prolactin levels, alleviating PMS symptoms (Meier et al., 2000; Tamagno, 2009). Chasteberry has also been shown to have hormone modulating properties in vitro that may play a role in regulating PMS symptoms (Girman et al., 2003; Lloyd and Hornsby, 2009). Weak binding affinity for the estrogen receptors and modulation of hormone sensitive genes have been reported in vitro (Liu et al., 2001, 2004; Hajirahimkhan et al., 2013a) as well as estrogenic effects 


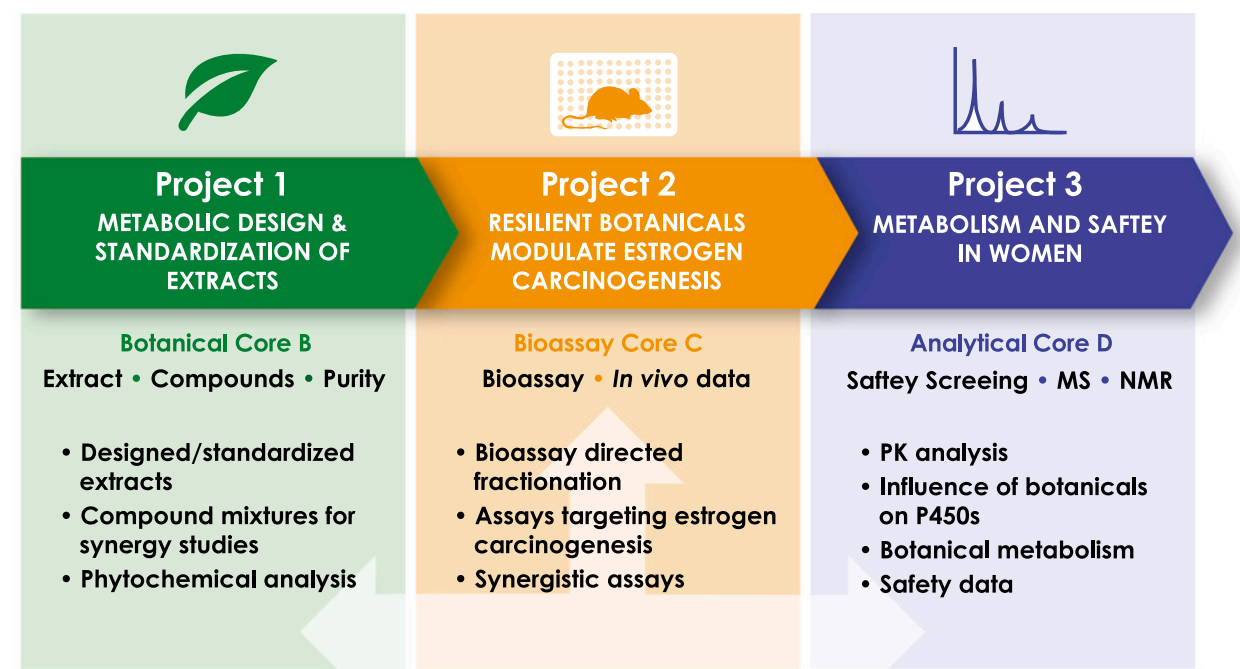

Fig. 12. The UIC/NIH Center for Botanical Dietary Supplements Research: Interaction of projects and cores.

on the uterus in rat models (Ibrahim et al., 2008). An alternative mechanism as an opiate receptor agonist has also been reported (Webster et al., 2006, 2011; Chen et al., 2011). A number of phytochemicals have been isolated from chasteberry including several flavonoids and linoleic acid, which could be responsible for the biological activities (Fig. 13) (Chen et al., 2011; Webster et al., 2011). Apigenin and penduletin in particular have been identified as weak $\mathrm{ER} \beta$-selective ligands (Fig. 13) (Jarry et al., 2003; Hu et al., 2007b), although apigenin is present in numerous plants. A small randomized, double-blind, placebo-controlled trial (67 Chinese women) did show significant efficacy of chasteberry compared with placebo in the treatment of moderate to severe PMS (Ma et al., 2010). Other small placebo controlled trials also suggested benefit; however, the placebo effect was large, which is often seen in clinical trials with effects on the CNS (Berger et al., 2000; Schellenberg, 2001, 2012; He et al., 2009; Zamani et al., 2012). These trials were of short duration and safety data were lacking. The German Commission E approved the use of chasteberry for PMS and chasteberry has the most convincing clinical data out of all of the botanicals for efficacy in the treatment of PMS (Freeman, 2010).

2. Angelica sinensis (Dong Quai). Dong quai or danggui is the dried root of Angelica sinensis, which is commonly used in traditional Chinese medicine to promote blood circulation and treat menstrual disorders such as dysmenorrhea as well as other women's health issues (Wu and Hsieh, 2011; Chen et al., 2013). It has been shown that a bioactive component of dong quai, $Z$-ligustilide (Fig. 10), inhibits the contraction of isolated rat uterus in a dose-dependent manner and improves microcirculation, suggesting it might be responsible for the antispasmodic effects of dong quai (Du et al., 2006). Ligustilide also has anti-inflammatory effects, which could contribute to the mechanisms of relief of menstrual symptoms (Su et al., 2011). Whether dong quai has estrogenic activity is controversial, and no estrogenic compounds have been isolated to date (Piersen, 2003; Hajirahimkhan et al., 2013a). Finally, there are no randomized, placebo-controlled, clinical trials evaluating the efficacy of dong quai for PMS.

3. Viburnum opulus and Viburnum prunifolium (Cramp Bark and Black Haw). Cramp bark and black haw are two species of viburnum that are often used as uterine relaxants and antispasmodics. Animal studies have suggested that both species have antispasmodic effects on the uterus and experiments with human uterine tissue also showed a relaxant effect (Jarboe et al., 1966; Nicholson et al., 1972; Cometa et al., 2009). Scopoletin could be responsible for the smooth muscle antispasmodic activity of viburnum (Fig. 14) (Jarboe et al., 1967; Nicholson et al., 1972). There are no randomized, placebo-controlled, clinical trials evaluating the efficacy of viburnum for PMS.

4. Zingiber officinale (Ginger). The rhizome of the ginger plant has been used for centuries as a spice and a condiment as well as for medicinal properties, particularly for anti-inflammatory and immune modulating properties (Ali et al., 2008). Ginger may also be effective at reducing PMS symptoms. More than 60 active compounds have been identified in ginger, including gingerols, shogaols, and zingerone (Fig. 15) (Baliga et al., 2011). Ginger and its bioactive compound zingerone have reported antispasmodic activities (Fig. 15) (van Andel et al., 2014; Ahmad et al., 2015). There are some reports of estrogenic activities for ginger, although other studies have shown no effect (Kim et al., 2008; Kumar et al., 2015). A small trial suggested that ginger (250 mg, 4 times daily, for three days from the start of the menstrual period) was as effective as NSAIDs at reducing period pain (Ozgoli et al., 2009). A few other small trials in young women have also shown positive effects for PMS (Kashefi et al., 2014, 2015). 


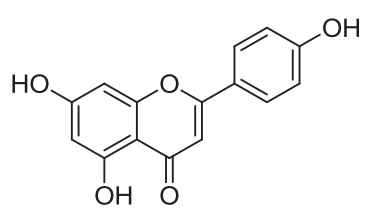

Apigenin

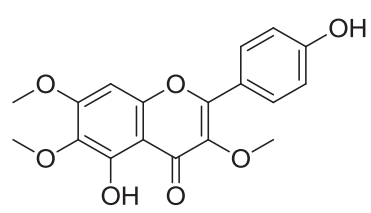

Penduletin

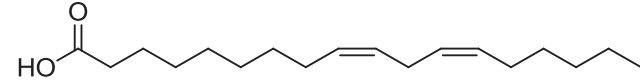

Linoleic acid

Fig. 13. Phytochemicals in chasteberry.

5. Valeriana officinalis (Valerian). The roots and rhizomes of valerian have been used for centuries primarily for tranquilizing/sedative properties as well as for PMS (Jarema, 2008). Over 150 phytochemicals have been identified in valerian, including pyridine alkaloids and organic acids such as valerenic acid (Fig. 16) (Patocka and Jaki, 2010). The antispasmodic effects of valerian have been demonstrated in vitro and in vivo (Occhiuto et al., 2009). A doubleblind, placebo-controlled, randomized clinical trial with 100 young women receiving $255 \mathrm{mg}$ three times daily for 3 days at the beginning of the menstrual cycle for two consecutive cycles showed a significant reduction in the severity of pain (Mirabi et al., 2011). No adverse effects were reported in the valerian-treated group, which supports literature evaluations of safety (Kelber et al., 2014).

6. Oenothera biennis (Evening Primrose). The oil from the seeds of evening primrose are high in fatty acids, including $\gamma$-linolenic acid (Montserrat-de la Paz et al., 2014) (Fig. 17). Polyphenolic compounds are also present, including gallic acid and chatechin, which could be responsible for antioxidant effects (Wettasinghe et al., 2002) (Fig. 17). A double-blind randomized clinical trial with 40 young women receiving either femicomfort (evening primrose, vitamins B6 and E) or placebo for two menstrual cycles concluded that evening primrose was effective in relieving PMS symptoms (Kashani et al., 2010). Another small randomized crossover study with 38 women showed no effect (Budeiri et al., 1996).

\section{B. Urinary Tract Infections}

Urinary tract infections (UTIs) are one of the most common bacterial infections in women, and women are 30 times more likely to develop UTIs compared with men (Little et al., 2010). The bacterium Escherichia coli is mainly responsible for most uncomplicated UTIs. Botanicals traditionally used to treat UTIs are reviewed below.

$$
\text { Scopoletin }
$$

Fig. 14. Phytochemical in cramp bark and black haw.
1. Vaccinium macrocarpon (Cranberry). Cranberry, which is the second most popular botanical sold in the US (Table 1), has a well-recognized medicinal use primarily in preventing UTIs (Yarnell, 2002; Rossi et al., 2010). The mechanism is generally thought to involve inhibition of adhesion of P-fimbriated (proteinaceous appendages) $E$. coli that firmly attach to uroepithelial cells by the type A proanthocyanidins in cranberry juice (Fig. 18) (Foo et al., 2000; Guay, 2009; Vasileiou et al., 2013). Three species of cranberry ( $V$. macrocarpon, $V$. oxycoccus, $V$. vitis-idaea) have different amounts and types of proanthocyanidins, which may explain the variable clinical data and further emphasizes the need for full chemical and biological standardization of cranberry extracts (Davidson et al., 2014). The Cochrane Collaboration previously recommended cranberry products for the prevention of UTIs in young and middle-aged women; however, questions of efficacy remain because of the heterogeneity in the clinical trial design and the lack of consensus on dosage, formulation, and time of use (Jepson et al., 2012). The most recent update suggests that cranberry juice is less effective than previously indicated, and they do not currently recommend cranberry juice for the prevention of UTIs. In addition, other cranberry preparations such as powders need to be fully standardized both chemically and biologically to the active compounds to ensure potency before being evaluated in clinical studies or recommended for use. The existing clinical trials suggest that cranberry is more effective at preventing rather than treating UTIs (Vasileiou et al., 2013); however, it is quite possible that the studies with $V$. macrocarpon in particular were under-dosed in the bioactive proanthocyanidins and placebo-controlled clinical trials that use standardized cranberry products are needed to establish efficacy (Davidson et al., 2014).

2. Arctostaphylos uva-ursi (Bearberry). Bearberry is a North American shrub, the leaves of which have ethnobotanical use for treating lower urinary tract infections (Yarnell, 2002). Bearberry contains tannins, which have astringent effects and can help to shrink and tighten mucous membranes, reducing inflammation and fighting infection. The antimicrobial compound could be arbutin, which is hydrolyzed in the gut to produce glucose and hydroquinone that can be further oxidized to para-quinone (Fig. 19) (de Arriba et al., 2013). Because hydroquinone/quinone is carcinogenic and tannins can cause nausea and 


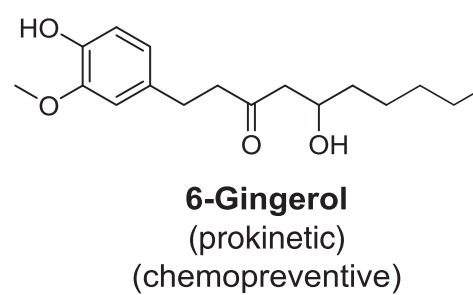<smiles>CCCCCC(O)CC(=O)CCc1ccc(O)c(OC)c1</smiles>

Phenoxy radical<smiles>CCCCCC=CC(=O)CCc1ccc(O)c(OC)c1</smiles>

6-Shogaol (chemopreventive)<smiles>COc1cc(CCC(C)=O)ccc1O</smiles>

Zingerone (antispasmodic) (chemopreventive)

Fig. 15. Phytochemicals in ginger and their metabolites.

vomiting, low doses of bearberry ( $800 \mathrm{mg}$ arbutin/day) are recommended for less than 1 week at a time. Currently there are no clinical trials on bearberry leaf alone to support the efficacy claims for treating UTIs.

\section{Botanicals Used during Pregnancy/Lactation}

\section{A. Nausea}

Nausea and vomiting during pregnancy can severely impact quality of life as well as pose significant health risks for some women. Botanicals traditionally used to relieve nausea associated with morning sickness are reviewed below.

1. Zingiber officinale (Ginger). Ginger might be an effective treatment of nausea associated with pregnancy, although the clinical data have been mixed (Kennedy et al., 2013; Giacosa et al., 2015; Marx et al., 2015). In general, ginger extracts have been shown to accelerate gastric emptying (promotility, prokinetic) and stimulate gastric antral contractions (Micklefield et al., 1999; Wu et al., 2008b). In addition, four randomized controlled clinical trials on the use of ginger for pregnancyinduced nausea and vomiting showed that orally administered ginger was significantly more effective than placebo at reducing frequency and intensity of nausea (Ding et al., 2013). The current literature suggests that ginger is safe in pregnant women; however, it is still not clear what the dose and length of treatment should be as well as the potential for drug/botanical interactions. GraviFrisk Ferrosan A/S (Søborg, Denmark), which contained a daily dose of $6 \mathrm{~g}$ dried ground ginger, was withdrawn from the Danish market in February 2008 because of inadequate scientific documentation of safety (Jacobsgaard, 2008). It is believed that the active compounds responsible for these biological effects are gingerols and shogaols (Fig. 15), which are cholinergic $\mathrm{M} 3$ and serotonergic $5-\mathrm{HT}_{3}$ receptor antagonists (Abdel-Aziz et al., 2006; Pertz et al., 2011). Gingerols, shogaols, and zingerone are metabolically unstable and are readily oxidized to phenoxy radicals and potentially quinones or quinone methides (Fig. 15), which could play a role in their chemopreventive activities (Gan et al., 2013; Mason and Thompson, 2014). The instability of these compounds could explain some of the varied activities reported in clinical trials. In general, the popular use of ginger for the relief of nausea during pregnancy is substantiated by a number of clinical trials that generally report efficacy relative to placebo and little toxicity (Dante et al., 2014). Similar results have been reported for ginger clinical trials for chemotherapy-induced nausea, motion sickness, and postoperative nausea (Dante et al., 2014).

\section{B. Lactation}

The most common reported reason for stopping breast feeding is the perception of inadequate milk production. A number of women have turned to herbal galactagogues to stimulate milk production despite the lack of documented efficacy and safety (Mortel and Mehta, 2013; Amer et al., 2015). Botanicals traditionally used to treat lactation disorders are reviewed below.

1. Trigonella foenum-graecum (Fenugreek). Fenugreek, which is a seed spice used to enhance flavor, color, and texture of food, also has several medicinal

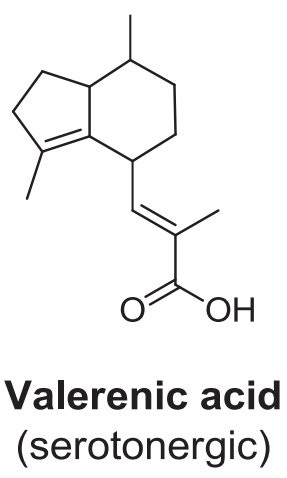

Fig. 16. Phytochemical in valerian. 

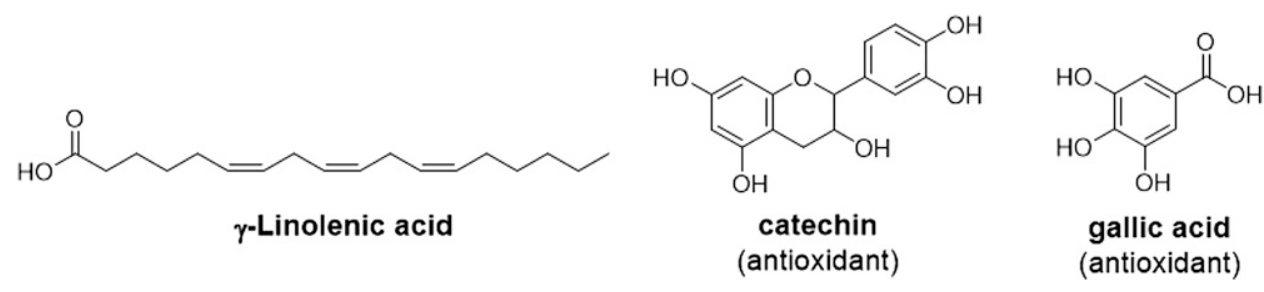

Fig. 17. Phytochemicals in evening primrose.

properties including galactagogue (lactation inducer) effects (Yadav and Baquer, 2014). It is the most commonly used herbal galactagogue, although there is conflicting reports of its efficacy (Mortel and Mehta, 2013). Fenugreek is thought to stimulate sweat production and because the breast is a modified sweat gland, it may stimulate breast milk production through this mechanism (Gabay, 2002). It has also been reported that fenugreek contains diosgenin (Fig. 20), which could modify the endocrine system enhancing milk production (Sreeja et al., 2010). Sugars of the flavonoids apigenin (Fig. 13) and luteolin (Fig. 20) have also been reported. In a well-designed clinical trial of 66 mother-infant pairs randomly assigned to three groups; fenugreek tea, apple tea, and control, maximum weight loss was significantly lower in the infants and milk volume was higher in the mothers receiving fenugreek tea (Turkyılmazet al., 2011). The research on the health benefits of fenugreek including its use as a galactagogue and its lack of side effects suggest potential as a dietary supplement (Yadav and Baquer, 2014).

2. Silybum marianum (Milk Thistle). The most common use of milk thistle supplements is for liver detoxification (Greenlee et al., 2007). Galactagogue activity of milk thistle has also been reported and its use is increasing (Forinash et al., 2012; Zapantis et al., 2012). It has been described in an in vivo study that milk thistle and specifically, silymarin (Fig. 21), a mixture of flavonolignans and some precursor flavonoids contained in milk thistle, increased prolactin levels in female rats and that this might lead to a galactagogue effect (Forinash et al., 2012). In an in vivo study with gestational pigs, feeding of $4 \mathrm{~g}$ silymarin twice daily for
20 days resulted in significant increase of circulating prolactin levels (Farmer et al., 2014). In addition, some parameters of mammary gland development changed through silymarin treatment; however, the increase in prolactin was not sufficient to have beneficial effects on mammary gland development in late gestation. The influence of silymarin on the amount of milk formation was not analyzed in this study. One placebo-controlled clinical trial analyzed silymarin's effectiveness as galactagogue in women (Forinash et al., 2012). Although an increase in milk formation was observed in this small clinical trial, this study had several limitations, which warrants further examination of milk thistle as a galactagogue in larger clinical investigations. The estrogenic activity of milk thistle and of silymarin was analyzed in different studies with varying results. Some studies describe weak estrogenic activities for silymarin and especially for its major flavonolignan, silybin B, and for quercetin and taxifolin (Pliskova et al., 2005) (Fig. 21 ). One 12 -week in vivo study showed that silymarin exerted estrogenic effects, such as increase of uterine weight and reduction of bone loss in ovariectomized rats (El-Shitany et al., 2010). A 1-week in vivo study using a lower dose of silymarin also showed an influence on osteoblast parameters but did not observe a uterotrophic effect for silymarin (Seidlova-Wuttke et al., 2003). Silymarin is reported to mainly exert estrogenic activity through the $\mathrm{ER} \beta$ receptor (Seidlova-Wuttke et al., 2003); however, it is controversial whether silibin B (El-Shitany et al., 2010) or other compounds contained in silymarin, such as taxifolin and quercetin, are responsible for the described ER $\beta$-selective effect (Powers and Setzer, 2015).<smiles>Oc1cc(O)c2c(c1)OC(c1ccc(O)c(O)c1)(c1ccc(O)c(O)c1)C(O)C2c1c(O)cc(O)c2c1OC(c1ccc(O)c(O)c1)C(O)C2</smiles>

Procyanidin A (antimicrobial)

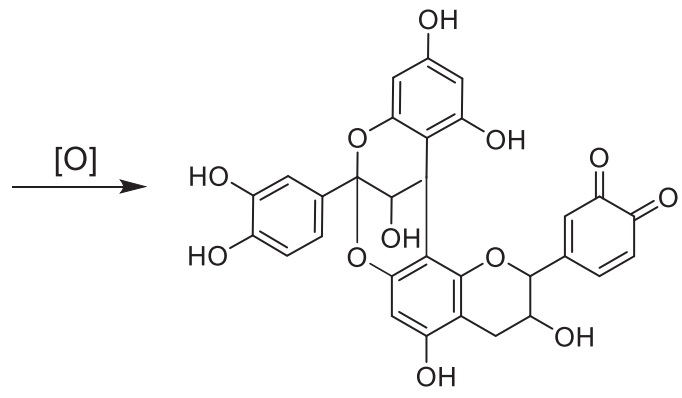

Procyanidin quinone

Fig. 18. Phytochemicals in cranberry. 


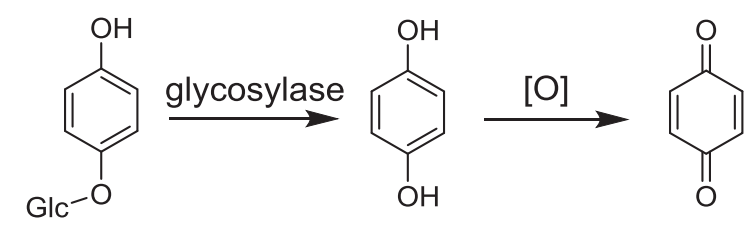

\section{Arbutin Hydroquinone Benzoquinone (antimicrobial)}

Fig. 19. Phytochemical in bearberry and its metabolites.

In general, the safety and efficacy of milk thistle as a galactagogue has not been analyzed in detail and further studies are warranted.

\section{Botanicals Used by Menopausal Women}

\section{A. Menopausal Symptoms}

The decrease in estrogen production at menopause leads to life-altering symptoms such as hot flashes and night sweats, cognitive instability, sleep disturbances, and vaginal atrophy (Nelson, 2008). These symptoms can be effectively managed by hormone therapy; however, as mentioned above, the results of the women's health initiative have led many women to complementary or alternative remedies such as the botanical dietary supplements (Table 1) (Borrelli and Ernst, 2010; Depypere and Comhaire, 2014; Poluzzi et al., 2014; Taylor, 2015). Botanicals traditionally used to treat menopausal symptoms are reviewed below.

1. Serotonergic Mechanism. Estrogen withdrawal during menopause results in a decline in the release of neurotransmitters, primarily norepinephrine and serotonin (5-hydroxytryptamine; 5 -HT), leading to a change in thermoregulation in the hypothalamus (Shanafelt et al., 2002) resulting in hot flashes and night sweats. Increase in the amount of serotonin and activating certain 5-HT receptors as well as inhibition of serotonin reuptake in synapses through blocking of serotonin transporters with prescription antidepressant SSRIs are viable approaches in preventing hot flashes (Fig. 22). However, there are also a number of undesirable outcomes such as nausea, sexual dysfunction, weight gain, and sleep disturbances associated with these remedies that have led women to consider natural botanicals with potential serotonergic efficacy

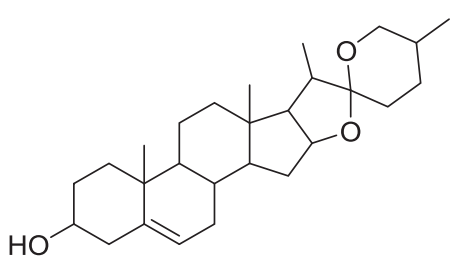

Diosgenin

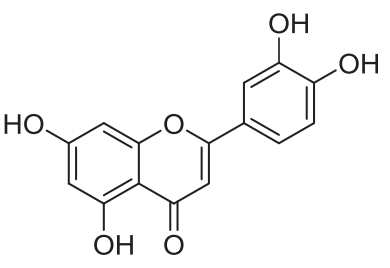

Luteolin
Fig. 20. Phytochemicals in fenugreek.
(Nachtigall, 2010; Kintscher, 2012; Hajirahimkhan et al., 2013a).

a. Actaea/Cimicifuga racemosa (black cohosh). Black cohosh is the most popular botanical for menopausal symptom relief in the United States (Taylor, 2012, 2015) (Table 1, rank number 4). The roots and rhizomes of black cohosh have been used by Native Americans for a variety of women's health issues for centuries. It is generally agreed that black cohosh is not estrogenic and the mechanism of action may involve modulation of the serotonergic system similar to the effects of antidepressants (Winterhoff et al., 2003; Borrelli and Ernst, 2008; Powell et al., 2008; Hajirahimkhan et al., 2013a; Wuttke et al., 2014). The active compounds in black cohosh are not known, although $N_{\omega}$-methylserotonin has been identified as a potential serotonin receptor agonist (Fig. 9) (Powell et al., 2008). The efficacy reports from black cohosh clinical trials have been highly variable (Shams et al., 2010). A meta-analysis of randomized placebo-controlled clinical trials concluded that of the six trials that demonstrated a significant improvement in the black cohosh group compared with placebo, a $26 \%$ improvement was calculated (95\% confidence interval, 11-40\%) (Shams et al., 2010). For example, Ruhlen et al. (2007) observed reduction of hot flashes in women taking a black cohosh extract containing $2.5 \%$ triterpenes for 12 weeks. The effect returned to baseline after a 12 -week washout period. The extract did not have any effect on serum estrogenic markers, trefoil factor 1 gene expression levels and cellular morphology in the women's nipple aspirate fluids, demonstrating no detectable estrogenic effect on the breast tissue (Ruhlen et al., 2007). More recent clinical trials have reported negative effects for black cohosh with no significant difference from placebo for relief of hot flashes. A black cohosh extract of $40 \mathrm{mg} /$ day was not superior to placebo for menopausal symptoms or improving quality of life scores in Thai women (Tanmahasamut et al., 2015). Similarly, a standardized extract of $125 \mathrm{mg} /$ day for 1 year also was not as effective as placebo (Geller et al., 2009). Given the popularity of black cohosh for relief of menopausal symptoms, larger placebo-controlled clinical trials are needed with fully standardized extracts before questions of efficacy and safety can be answered.

$b$. Valeriana officinalis (valerian). Extracts of valerian roots are widely used for inducing sleep and improving sleep quality, suggesting they might be effective for menopausal symptoms especially night sweats (Bent et al., 2006). Serotonergic activity has also been reported for valerian extracts, and valerenic acid has been identified as a potential partial agonist of the $5-\mathrm{HT}_{5 \mathrm{~A}}$ (Fig. 16) (Dietz et al., 2005b). In a double-blind placebo-controlled clinical trial, 68 women receiving $255 \mathrm{mg}$ valerian capsules three times daily for 8 weeks showed a significant reduction in hot flash frequency and intensity (Mirabi and Mojab, 2013). Another randomized placebo-controlled trial with a mixture of valerian 
A<smiles>COc1cc(C2Oc3cc(C4Oc5cc(O)cc(O)c5C(=O)C4O)ccc3OC2CO)ccc1O</smiles><smiles>O=C1c2c(O)cc(O)cc2OC(c2ccc(O)c(O)c2)C1O</smiles><smiles></smiles>

Silybin A, B

(estrogenic)

Taxifolin

Quercetin

B<smiles>COc1cc([C@H]2Oc3ccc(-c4oc5cc(O)cc(O)c5c(=O)c4O)cc3O[C@H]2CO)ccc1O</smiles>

\section{2,3-Dehydrosilybin}

(P450 1A1 inhibitor)

Fig. 21. Phytochemicals in milk thistle.

and lemon balm (100 women, 50-60 years, $160 \mathrm{mg} /$ day valerian) showed a significant reduction in sleep disorders with the treated group (Taavoni et al., 2013). A recent 8-week study on 60 postmenopausal women (45-60 years old) showed a significant improvement in menopausal symptoms in women receiving $530 \mathrm{mg}$ of valerian root capsules twice a day (Izadjoo et al., 2015). No adverse effects were reported in these trials, suggesting valerian supplements at these doses are safe.

2. Estrogenic Mechanism. Botanicals with estrogenic activity can mimic the effects of endogenous estrogens lost during menopause (Hajirahimkhan et al., 2013a). Estrogens often activate biological responses through binding to estrogen receptors (ERs) including ER $\alpha$ and $\mathrm{ER} \beta$, followed by dimerization of ERs and interaction with

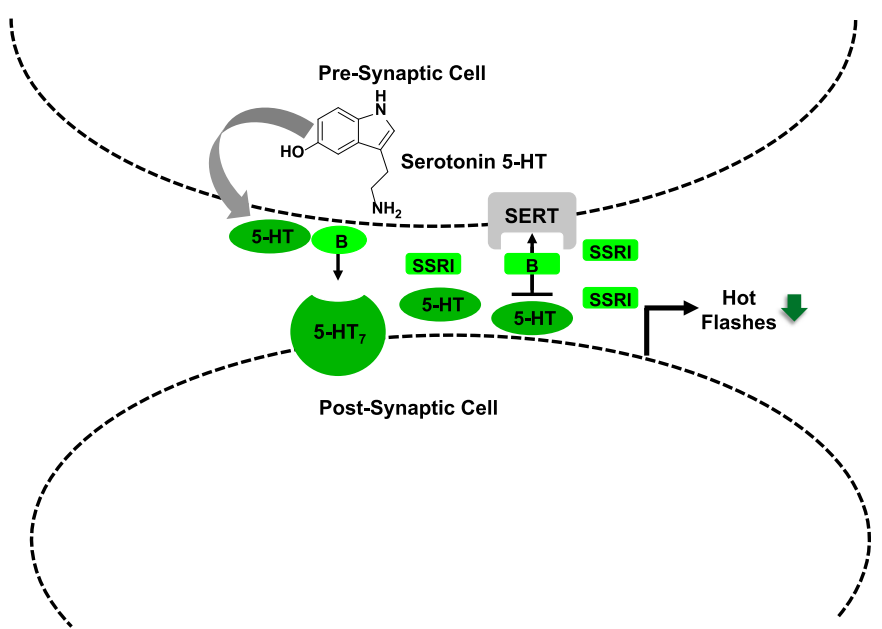

Fig. 22. Serotonergic mechanism. In synapses, botanicals can directly act on the serotonin receptors (5-HT receptors) or reduce serotonin reuptake through inhibiting serotonin transporters (SERTs) to elicit a reduction in menopausal symptoms such as hot flashes. estrogen responsive elements (EREs) at the promoter of the estrogen responsive genes, thus activating transcription and generating estrogenic effects that are crucial for normal physiological functions (Fig. 23) (Nilsson et al., 2001; Bjornstrom and Sjoberg, 2005; Ellmann et al., 2009; Jia et al., 2015). It is believed that $\mathrm{ER} \alpha$ induction is associated with the proliferative effects of estrogens, whereas $\mathrm{ER} \beta$ opposes the ER $\alpha$-dependent proliferation (Deroo and Buensuceso, 2010; Shanle and Xu, 2010; Thomas and Gustafsson, 2011; Jia et al., 2015). Botanicals with estrogenic effects, particularly the ones with $\mathrm{ER} \beta$ selectivity, may offer safer alternatives to conventional prescription HT regimens.

\section{a. Isoflavones.}

$i$. Glycine max (Soy). Soy is one of the major sources of protein in Asian diets in particular (Low Dog, 2005; Taylor, 2015). Isoflavones in soy foods are mainly present as glycosides (i.e., genistin, daidzin), which are hydrolyzed by $\beta$-glucosidases in the intestine forming the estrogenic $\mathrm{ER} \beta$-selective aglycones, genistein and daidzein (Fig. 24) (Lin and Giusti, 2005; Lopez-Gutierrez et al., 2014; Taylor, 2015). It has also been reported that daidzein metabolism by the intestinal microflora results in the formation of $S$-equol, which has better estrogenic activity and a higher affinity for ER $\beta$ than daidzein (Setchell et al., 2005; Jiang et al., 2013) (Fig. 24). Different anaerobic bacteria can reduce daidzein to $S$-equol through three different reductase enzymes and one dihydrodaidzein racemase (Shimada et al., 2011; Lee et al., 2016). Genetic polymorphism in equol production has been demonstrated, which in part may explain the variability in relieving menopausal symptoms (Hong et al., 2012).

Most clinical trials have used dosages ranging from 50 to $100 \mathrm{mg} /$ day isoflavones (Low Dog, 2005). The 


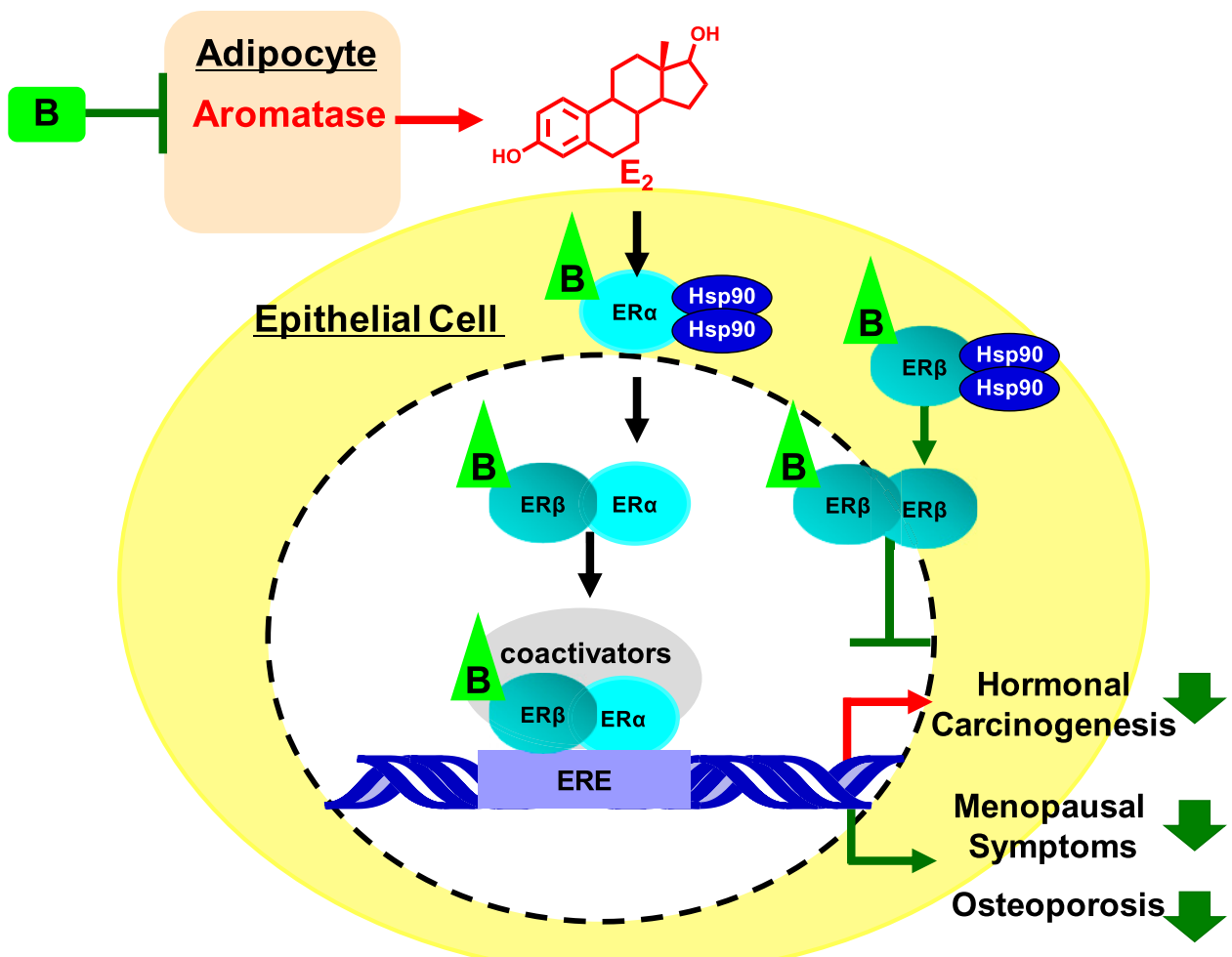

Fig. 23. Hormonal pathway. $\mathrm{ER} \alpha$ and $\mathrm{ER} \beta$ are activated by estrogens or phytoestrogens that cause dimerization of these receptors and translocation to the nucleus. Activation of ERE-responsive genes by ER $\alpha$ increases proliferation yet reduces menopausal symptoms and osteoporosis. On the other hand, ER $\beta$ decreases proliferation, while also decreasing menopausal symptoms and osteoporosis. Additionally, many botanicals reportedly decrease aromatase expression/activity to reduce synthesis of estrogen. This figure was simplified to represent only the classic estrogen signaling pathway.

North American Menopause society has concluded that soy-based isoflavones are modestly effective in relieving menopausal symptoms (North American Menopause Society, 2011). A meta-analysis of the use of soy dietary supplements for hot flashes showed a statistically significant decrease in hot flashes, although the clinical efficacy is questionable (Bolanos et al., 2010). A review published by the Cochrane Collaborative on 43 randomized controlled trials concluded that there was no conclusive evidence that soy supplements reduced menopausal hot flashes (Lethaby et al., 2013). Fortunately, no estrogenic effects on the endometrium or vagina were reported in these studies with up to 2 years of use. The poor bioavailability of genistein and daidzein from soy may explain the variable and relatively modest if any effects on menopausal symptoms (Yang et al., 2012; RodriguezMorato et al., 2015).

ii. Trifolium pratense (Red Clover). Unlike soy, the major isoflavones in red clover are the proestrogens biochanin A and formononetin (Fig. 24) (Booth et al., 2006). These methoxy precursors are demethylated by cytochrome P450 isozymes in the intestine and liver to the estrogenic $\mathrm{ER} \beta$-selective genistein and daidzein, respectively (Tolleson et al., 2002). These data suggest that red clover isoflavones might have higher bioavailability compared with soy and could explain why the red clover clinical trials tend to show more efficacy for menopausal symptoms compared with those evaluating soy products
(Piersen et al., 2004; Thomas et al., 2014). A meta-analysis of four clinical trials using the red clover extract marketed as Promensil (Novogen Ltd, Hornsy, Australia) showed a slight but statistically significant reduction in hot flashes (Lethaby et al., 2013). In addition, there was no increase in endometrial thickness or breast density, suggesting a lack of estrogenic activity in hormone-sensitive tissues. A recent trial of 72 women randomly divided between placebo and $40 \mathrm{mg}$ dried red clover daily for 12 weeks showed a significant reduction in menopausal symptoms as measured by the menopause rating scale (Shakeri et al., 2015). Other clinical trials have not shown a significant difference from placebo, particularly for hot flash relief (Geller et al., 2009; Hall et al., 2011).

iii. Pueraria lobata (Kudzu). Kudzu is used in traditional Chinese medicine for the treatment of menopausal symptoms (Hajirahimkhan et al., 2013a). The major isoflavone in kudzu is puerarin, which is the C8-glucoside of daidzein (Guerra et al., 2000; Delmonte et al., 2006) (Fig. 24). Puerarin is metabolized to daidzein by intestinal bacteria (Park et al., 2006; Nakamura et al., 2011). The same isoflavonoids found in red clover and soy are also found in kudzu in lower amounts (Delmonte et al., 2006; Zhang et al., 2009). A randomized controlled clinical trial (127 women, 50-65 years old, 3 months) receiving kudzu standardized to $100 \mathrm{mg}$ isoflavones, HT, or no treatment showed no significant changes compared with control (Woo et al., 2003; Ulbricht et al., 2015). 


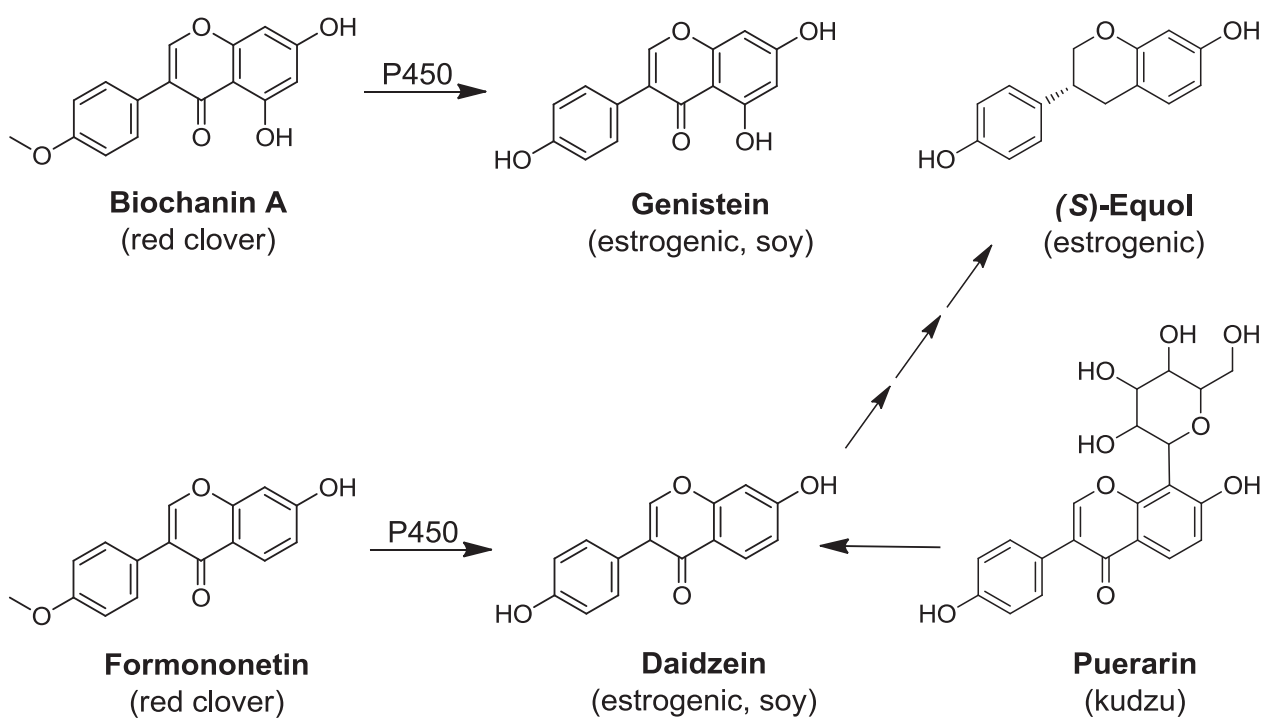

Fig. 24. Isoflavones in red clover, soy, and kudzu. Genistein and daidzein are both found in soy and red clover; however, they are significantly more abundant in soy. Daidzein is metabolized by the intestinal microflora to $S$-equol.

iv. Eriosema laurentii (Guinea-Bissau). Guinea-bissau is a botanical found in Cameroon traditionally used for infertility and menopausal symptoms (Ntie-Kang et al., 2013). Phytoestrogens isolated from guinea-bissau include $\mathrm{ER} \alpha$-selective lupinalbin A (Fig. $25)$ and $\operatorname{ER} \beta$-selective genistein (Fig. 24) (Ateba et al., 2014). No clinical trials have been reported on the use of guinea-bissau for menopausal symptoms.

b. Humulus lupulus (hops). The strobiles of hops are often added to beer to produce bitter, tart, hoppy flavors. Hop extracts are also a popular botanical for sleepinducing effects, especially in Europe (Blumenthal and German Federal Institute for Drugs and Medical Devices Commission, 2000; Zanoli and Zavatti, 2008; Salter and Brownie, 2010). Hop extracts are also present in some dietary supplements for managing menopausal symptoms (Taylor, 2012). The most potent phytoestrogen in hops is the $\mathrm{ER} \alpha$-selective agonist 8-prenylnaringenin (8-PN), which is in part formed from the chemopreventive isoflavone xanthohumol through cyclization and P450 catalyzed demethylation as shown in Fig. 26 as well as through metabolism of isoxanthohumol by microbiota (Nikolic et al., 2005; Chadwick et al., 2006; Possemiers et al., 2006). A few clinical trials have been published evaluating the efficacy of hops extracts on menopausal symptoms (Taylor, 2015). A randomized placebo controlled trial with 67 menopausal women over 12 weeks receiving hops extract standardized to 100 or $250 \mu \mathrm{g}$ 8-PN or placebo showed significant effects at 6 weeks for the lower dose. However, no effect was observed at higher doses, and the lower dose was not significantly different from placebo after 12 weeks as measured by the Kupperman index and quality of life questionnaire (Heyerick et al., 2006). The authors noted the relatively low effective dose of $\mathrm{ER} \alpha$-selective 8-PN, which is 100 -fold more potent than the ER $\beta$-selective isoflavones genistein and daidzein (Milligan et al., 1999;
Overk et al., 2008). However, given the potent ER $\alpha$ agonistic activity of 8-PN in hops extracts, safety with respect to the effect on the endometrium and other hormone sensitive tissues needs further study. In a smaller study (randomized, double-blind, placebocontrolled, crossover study, 36 menopausal women), women receiving either placebo or hops extract standardized to 8-PN for 8 weeks and then switching treatments for 8 weeks showed weak efficacy of the extract over placebo when the extract was taken after the placebo treatment until week 16 (Erkkola et al., 2010). However, these results might not be robust, considering the small sample size and large number of variables included in the extensive statistical analysis (Taylor, 2015). A more recent trial using a daily dose of $500 \mathrm{mg}$ hops in tablet form versus placebo (120 women, 90 days) showed a statistically significant reduction in menopausal symptoms and a dramatic reduction in hot flashes (Aghamiri et al., 2016). However, there are several problems with this trial including, the lack of standardization of the extract, the recruitment of women from multiple sites, and the lack of a placebo effect. No adverse effects were reported in any of these trials. More clinical trials are needed before the safety and efficacy of hops extracts for menopausal symptom relief can be established.

c. Glycyrrhiza species (licorice). Licorice is a widely used plant, mainly as a sweetening agent in tobacco, in food and beverages, and in toothpastes. It consists of more

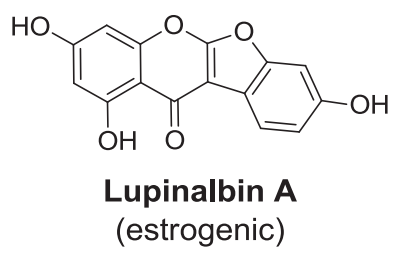

Fig. 25. Isoflavone in guinea-bissau. 
Dietz et al.<smiles>CC(C)=CCc1c(O)cc(OCC(C)(C)C)c2c1OC(c1ccc(O)cc1)CC2=O</smiles><smiles>COc1cc(O)c(CC=C(C)C)c(O)c1C(=O)/C=C/c1ccc(O)cc1</smiles>

Xanthohumol (XH) (chemopreventive)<smiles>CC(C)=CCc1c(O)cc(O)c2c1OC(c1ccc(O)cc1)CC2=O</smiles><smiles>CC(C)=CCc1c(O)cc(O)c(C(=O)/C=C/c2ccc(O)cc2)c1O</smiles>

Desmethylxanthohumol (DMX)<smiles>CC(C)=CCc1c(O)cc2c(c1O)C(=O)CC(c1ccc(O)cc1)O2</smiles>

Fig. 26. Phytochemicals in hops.

than 30 species, from which a few have been studied for several biological effects (Asl and Hosseinzadeh, 2008; Hajirahimkhan et al., 2013b). Licorice is commonly present in menopausal botanical supplements in the United States (Taylor, 2012). There are three pharmacopeial licorice species, Glycyrrhiza uralensis, G. glabra, and G. inflata; however, the specific species used is often not identified or mislabeled in supplements. The estrogenic activities of different licorice species are variable and likely depend on the type and amounts of bioactive compounds, emphasizing the importance of fully standardizing extracts using chemical, genetic, and biological methods (Liu et al., 2001; Hajirahimkhan et al., 2013a). After evaluating a large number of licorice samples that were local to certain regions of the world Kondo et al. (2007a) reported that $G$. uralensis and G. glabra had the highest and the lowest amounts of the phytoestrogen liquiritigenin (Fig. 27), respectively. Liquiritigenin was reported to be a selective ER $\beta$ agonist in the ER binding assay and in ER $\beta$-ERE-luciferase activity assay in U2OS cells (Mersereau et al., 2008). In vivo liquiritigenin did not enhance proliferation of MCF-7 $(\mathrm{ER} \alpha+)$ xenograft or induce uterine weight in nude mice, suggesting liquiritigenin is unlikely to affect hormone sensitive tissues (Mersereau et al., 2008). Isoliquiritigenin, the precursor chalcone of liquiritigenin was also shown to exhibit estrogenic effects (Fig. 27) (Maggiolini et al., 2002). However, the conversion of isoliquiritigenin to liquiritigenin could play a significant role in exerting the observed activity (Hajirahimkhan et al., 2013b). There have been very few clinical studies evaluating licorice for the treatment of menopausal symptoms. In a double-blind placebo-controlled trial, a significant drop in hot flashes was reported with 90 women receiving licorice $330 \mathrm{mg} /$ day or placebo for 8 weeks (Nahidi et al., 2012). However, no mention about the species of licorice extract was available and no placebo effect was observed. Another recent trial with six women compared the efficacy of licorice extracts $(1140 \mathrm{mg} /$ day $)$ 
A

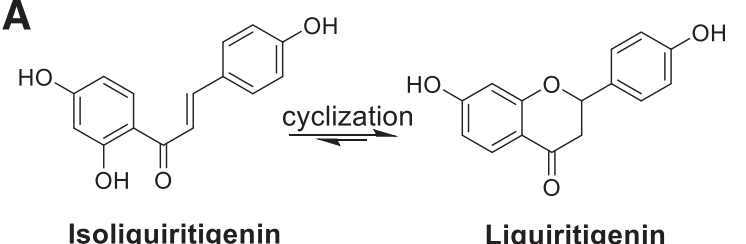

Isoliquiritigenin

Liquiritigenin

(chemopreventive)

(estrogenic)

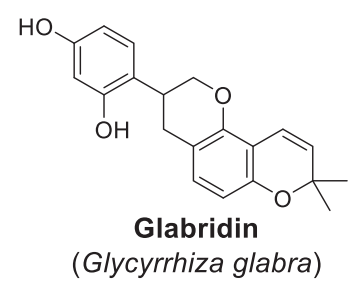

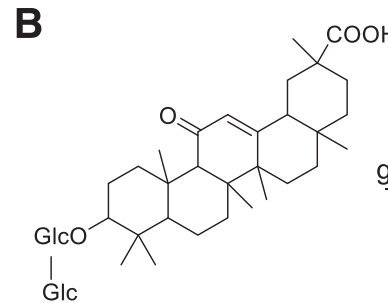

Glycyrrhizinic acid

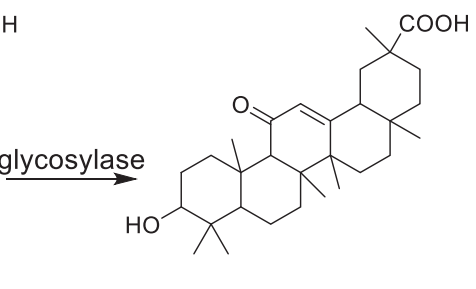

Glycyrrhetinic acid

(chemopreventive)

(AhR antagonist)

(Glycyrrhiza inflata)

Fig. 27. Phytochemicals in licorice.

for 90 days with traditional HT for hot flash relief claiming similar efficacy (Menati et al., 2014). Both trials have significant problems ranging from small size, lack of extract standardization, self-reporting surveys, lack of placebo group, and lack of placebo effect, which can be as high as 50\% in menopause trials. More in-depth studies are needed to define the efficacy and safety of licorice for menopausal symptoms.

$d$. Rheum rhaponticum (rhubarb). Rhubarb is also a common herb for menopausal symptom relief, particularly in Germany (Vollmer et al., 2010; Taylor, 2012; 2015). A special extract prepared from the roots of Rheum rhaponticum called ERr731 [marketed under the trade name Phytoestrol N (Mueller Goeppingen, Goeppingen, Germany)] has been in use in Germany for decades without any significant side effects such as endometrial hyperplasia, spotting, or breakthrough breeding (Wober et al., 2007). This extract mainly consists of rhaponticin and desoxyrhaponticin, which are converted to the resveratrol-like aglycones rhapontigenin and desoxyrhapontigenin by the microbiome and glycosylases (Fig. 28) (Kim et al., 2000; Wober et al., 2007). Weak $\mathrm{ER} \beta$ activity has been measured for both the extract and the aglycones rhapontigenin and desoxyrhapontigenin in transfected HEC-1B adenocarcinoma cells and U2OS cells (Moller et al., 2007; Wober et al., 2007). Rhapontigenin is more active than desoxyrhapontigenin and it is tempting to suggest that $\mathrm{P} 450$-catalyzed $O$-demethylation giving the resveratrol catechol piceatannol might be responsible for the estrogenic activity (Wober et al., 2007). It has been shown that piceatannol binds estrogen receptors and stimulates the growth of estrogen-dependent cancer cells
(Piotrowska et al., 2012). The efficacy and safety of ERr731 for menopausal symptoms has been studied in a number of double-blind placebo-controlled trials with positive results (Heger et al., 2006; Kaszkin-Bettag et al., 2008; Hasper et al., 2009). All these trials show that the rhubarb extract was well tolerated both for short duration (12 weeks) as well as long term (up to 2 years), successfully decreased the Menopause Rating Scale, and increased quality of life.

$e$. Vitex agnus-castus (chasteberry). Chasteberry is also a popular botanical added to botanical supplements for menopause (van Die et al., 2009; Taylor, 2012). It was shown to have a weak binding affinity for ERs and no estrogen-sensitive alkaline phosphatase induction in Ishikawa cells; however, it did induce the estrogensensitive gene $P g R$ (progesterone receptor gene) mRNA in this cell line (Liu et al., 2001). Linoleic acid (Fig. 13) was isolated as the "active" estrogenic constituent of chasteberry based on bioassay guided fractionation of the crude extract using the ER binding assay (Liu et al., 2004). However, linoleic acid is a fatty acid and could form nonspecific binding to ERs and progesterone receptor proteins, leading to false-positive results. Moreover, induction of $P g R$ could be related to possible progestogenic activity of the extract and not necessarily its $\mathrm{ER} \alpha$-dependent effects. It was shown that four different species of chasteberry enhance MCF-7 cell proliferation (Hu et al., 2007b). In addition, the essential oils of Vitex rotundifolia, which is mainly composed of linoleic acid, strongly enhanced MCF-7 cell proliferation, and the effect could be blocked by the pure antiestrogen, ICI 182,780 (7 $\alpha, 17 \beta$-[9-[(4,4,5,5,5Pentafluoropentyl)sulfinyl]nonyl]estra-1,3,5(10)-triene-3, 
<smiles>COc1cc(O)cc(/C=C/c2cc(O)cc(/C=C/c3cc(/C=C/c4ccc(OC)c(O)c4)ccc3OC)c2)c1</smiles>

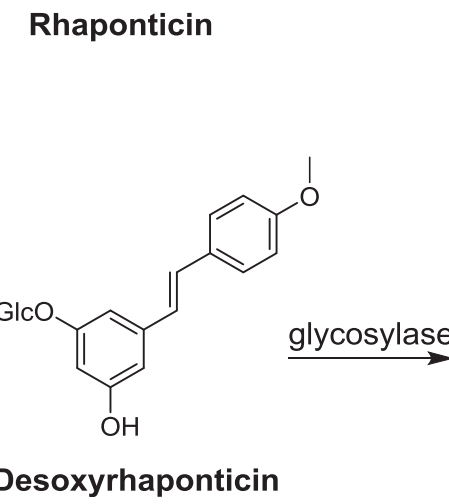

Rhapontigenin

Piceatannol

(estrogenic)<smiles>COc1ccc(/C=C/c2cc(O)cc(O)c2)cc1</smiles>

Desoxyrhapontigenin

Resveratrol

Fig. 28. Phytochemicals in rhubarb and their metabolites.

17-diol) (Hu et al., 2007a). Similarly, Ibrahim et al. (2008) reported that an ethanolic extract of chasteberry enhanced uterine weight in Sprague-Dawley rats. These data suggest that chasteberry extracts have ER $\alpha$-dependent estrogenic activity. It was also shown that chasteberry extract selectively bound $\mathrm{ER} \beta$ and bioassay guided fractionation of the crude extract resulted in the isolation of the weak ER $\beta$ ligand apigenin (Fig. 13) (Jarry et al., 2003). The ER $\beta$ selectivity of apigenin was also shown in competitive binding assays in addition to general estrogenic activity in a yeast based and MCF-7 cell growth assays (Choi et al., 2008). Despite the popularity of chasteberry with herbalists and its use as a component of menopausal formulations with other botanicals, there are no clinical trials with chasteberry as a sole agent for the relief of menopausal symptoms (van Die et al., 2009). However, the studies with combinations of botanicals including chasteberry are promising, suggesting further investigation of Vitex agnus-castus for menopausal symptom relief (van Die et al., 2009).

f. Linum usitatissimum (flaxseed). Flaxseed is a major source of lignans that are metabolized by the intestinal flora into the weakly active phytoestrogens enterolactone and enterodiol (Fig. 29) (Dew and Williamson, 2013). Lignans reside in cell walls and are not bioavailable without crushing, and as a result the best sources for women are flaxseed flour and flaxseed meal. Flaxseed oil is a good source of $\alpha$-linolenic acid but does not contain lignans (Taylor, 2015). In a randomized placebo-controlled clinical trial (90 women, $1 \mathrm{~g} /$ day flaxseed extract), modest but significant effects were observed in self-reporting relief of menopausal symptoms (Colli et al., 2012). No adverse effects related to flaxseed treatment were reported. A meta-analysis of recent randomized clinical trials examining the efficacy of flaxseed for menopausal symptom relief concluded that there is little evidence to support the use of this dietary supplement for menopause or for bone health (Dew and Williamson, 2013).

g. Epimedium species (horny goat weed). Horny goat weed originating from different Epimedium species has been used in traditional Chinese medicine for over 2000 years to enhance libido in men and women as well as for symptoms of menopause and PMS (Ma et al., 2011). The flavonoid icariin can be metabolized to icaritin and desmethylicaritin, which is likely responsible for the mostly $\mathrm{ER} \alpha$-selective estrogenic bioactivities (Fig. 30) (Wang and Lou, 2004; Cao et al., 2012; Xiao et al., 2014). These prenylated flavonoids are structurally similar to the potent ER $\alpha$ estrogen 8-PN in hops (Fig. 26, see above). One clinical trial showed that an extract of horny goat weed could increase estrogen levels and improve lipid profiles in postmenopausal women without any significant side effects (Yan et al., 2008). No clinical trials using horny goat weed for menopausal symptoms have been reported.

$h$. Lepidium meyenii (maca). Maca has been used in South America for centuries for infertility and female

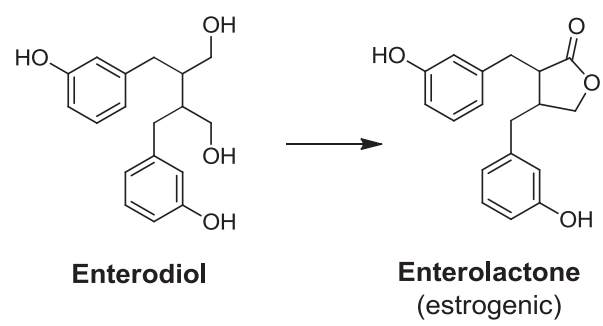

Fig. 29. Phytochemicals in flaxseed. 
hormone balance, especially for menopausal symptoms (Lee et al., 2011b; Gonzales, 2012; Taylor, 2015). Maca extracts have been shown to have estrogenic activity by increasing MCF-7 cell proliferation (Valentova et al., 2006), although the active phytoestrogens are not known. Maca extracts contain a number of phytosterols including beta-sitosterol and campesterol (Fig. 31) as well as free fatty acids including linolenic acid (Fig. 17) (Dini et al., 1994). A systematic review of clinical trials concluded that there was limited evidence for the effectiveness of maca for relief of menopausal symptoms; however, the sample size, the number of trials, and the quality of the trials were too limited to establish firm conclusions on efficacy and safety (Lee et al., 2011b).

$i$. Oenothera biennis (evening primrose). Evening primrose oil is a good source of omega-6 essential fatty acids including $\gamma$-linolenic acid (Fig. 17). In a randomized placebo-controlled clinical trial (56 menopausal women), a significant improvement in the severity of menopausal hot flashes was reported in the evening primrose (500 mg/day, 6 weeks) group (Farzaneh et al., 2013). A systematic review of evening primrose oil for menopausal symptoms suggests that although the herb is well tolerated, efficacy is not clinically significant (Bayles and Usatine, 2009).

$j$. Medicago sativa (alfalfa). Alfalfa is another botanical that is a good source of isoflavones. The coumestan derivative coumestrol (Fig. 32) is the major estrogenic phytochemical, although the licorice flavonoids liquiritigenin and isoliquirtigenin have also been detected (Fig. 27) (Hong et al., 2011). No clinical trials for menopause using alfalfa alone have been reported.

\section{Unknown Mechanism.}

a. Dioscorea villosa (wild yam). Wild yam contains diosgenin, which is used in the manufacturing of synthetic steroids (Taylor, 2015). Diosgenin can be chemically converted to progesterone, although there is no biological pathway for the conversion in vivo (Fig. 20). When given as a cream, there was no significant effect on menopausal symptoms in 23 women after 3 months compared with placebo (Komesaroff et al., 2001). No significant adverse effects were reported. The current recommendations are that yam creams are ineffective for menopause and because many contain adulterants they should be avoided (Taylor, 2015).

b. Angelica sinensis (dong quai). The roots of Angelica sinensis have been used for restoring female hormonal balance in traditional Chinese medicine for centuries (Hook, 2014). The estrogenic activity of dong quai is controversial (Liu et al., 2001; Lau et al., 2005; Circosta et al., 2006). For example, it was shown that dong quai had proliferative effects in MCF-7 (ER $\alpha+)$ cells, whereas it did not induce $\mathrm{ER} \alpha / \mathrm{ER} \beta$-dependent luciferase transcription in transfected HeLa cells and did not show uterotrophic activity in CD-1 mice (Amato et al., 2002). However, enhanced uterine weight, change in vaginal cytology, and reduced luteinizing hormone levels in female Wistar rats treated with an ethanolic extract of dong quai have been shown (Circosta et al., 2006). Similarly, cellbased studies showed controversial results. Dong quai methanolic extracts did not show binding affinity to ERs, induce alkaline phosphatase activity in Ishikawa cells, or induce transcription of estrogen responsive genes (Liu et al., 2001). Moreover, an ethanolic extract of dong quai did not exhibit estrogenic activity in a yeast-based assay over the concentration range of $0.1-1000 \mu \mathrm{g} / \mathrm{ml}$ (Zhang et al., 2005). It has been shown that the lipophilic fraction of a methanolic extract (rich in ligustilide, Fig. 10) of dong quai at $20 \mu \mathrm{g} / \mathrm{ml}$ significantly inhibited alkaline phosphatase induction in the presence of estradiol in Ishikawa cells, suggesting an antiestrogenic potential (Godecke et al., 2012). To date, there have not been any reports of a purified compound, which could be responsible for the observed estrogenic/antiestrogenic activities of dong quai. These studies suggest that further research with welldefined extracts are needed to delineate the estrogenic/ antiestrogenic potential of dong quai as well as the active compound. One reason for the contradictory results regarding the estrogenic activities of dong quai could be associated with the instability of its phthalide fractions, in particular Z-ligustilide as discussed previously (Schinkovitz et al., 2007). In traditional Chinese medicine, dong quai is usually combined with other botanicals; therefore very few clinical trials on dong quai alone have been reported. One study with 71 postmenopausal women randomized to $4.5 \mathrm{~g}$ dong quai/day or placebo

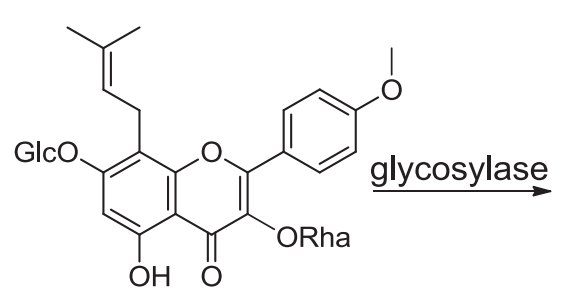

Icariin

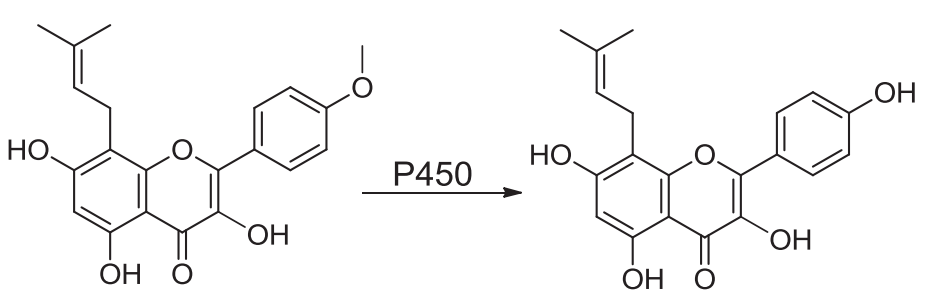

Icaritin 8-prenylkaempferol or
desmethylicaritin

(estrogenic)

Fig. 30. Phytochemicals in horny goat weed. 

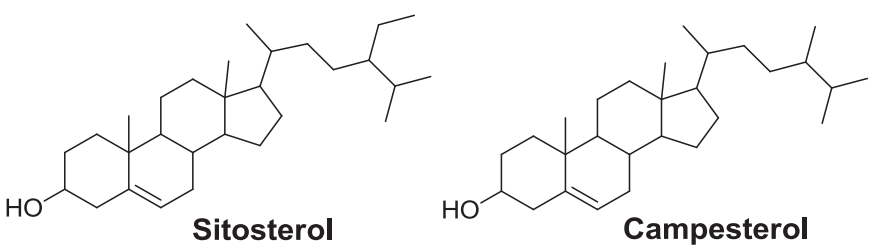

Fig. 31. Phytochemicals in maca.

for 24 weeks showed no effect on menopausal symptoms (Hirata et al., 1997). No estrogenic effects on the endometrium or vagina were observed and no significant side effects were reported in this trial. Conclusive data on the relative efficacy of dong quai for menopausal symptoms are currently unavailable.

\section{B. Osteoporosis}

A consequence of low estrogen levels at menopause is an increase in osteoporosis risk. Botanicals, which may protect against osteoporosis, are reviewed below. In general, there is insufficient evidence to make recommendations on the effect of botanicals on bone health.

1. Isoflavones. Because most isoflavones are $\mathrm{ER} \beta$ selective ligands, it should be possible to exploit this selectivity and target mainly bone cells without adversely affecting hormone-sensitive tissues such as the uterus and the breast. Genistein (Fig. 24) has been shown to stimulate bone formation, inhibit bone resorption, and prevent bone loss in ovariectomized rat models (Taylor et al., 2009; Fu et al., 2014). It has been demonstrated in a few randomized, double-blind placebocontrolled studies that genistein $(54 \mathrm{mg} /$ day for 1 or 2 years) was effective in preventing bone loss in postmenopausal women (Morabito et al., 2002; Marini et al., 2007). Favorable effects have been demonstrated in other clinical studies measuring bone mass density and bone turnover markers; however, the long-term effects on prevention of fractures have not been established (Lagari and Levis, 2014; Poluzzi et al., 2014). The data suggest that diets rich in isoflavones are beneficial for bone health in the long term, although the magnitude of the effects and the exact mechanism of how isoflavones could protect bones have not been determined. There is also evidence to suggest that the positive effects of isoflavones on bone health could vary as a function of age. Isoflavones could be more effective in younger women before loss of estrogen receptors

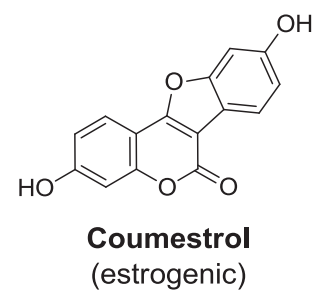

Fig. 32. Phytochemical in alfalfa. observed in postmenopause (Reinwald and Weaver, 2006). Most clinical trials with soy proteins and enriched soy isoflavones were not effective in decreasing bone loss, in particular at common fracture sites in healthy perimenopausal and postmenopausal Western women (Alekel et al., 2010). Different studies have also analyzed red clover products for prevention of osteoporosis. A patented aqueous and fermented red clover extract standardized to $37.1 \mathrm{mg}$ isoflavones per day or a placebo solution were administered to women suffering from menopausal symptoms in a 3-month, randomized, double-blind and parallel designed trial (Thorup et al., 2015). A significant decline in the bone mineral density of the lumbar spine (lower back) in the placebo group but not in the red clover group was observed, suggesting a positive effect on bone health by the red clover extract. However, it has to be noted that the actual changes were small and that the differences could also be due to the variability in bone density scanning. Studies with bisphosphonate therapy showed that reliable treatment effects can be best observed after 3 years, because the within-person variations differ widely with dualenergy X-ray absorptiometry measurement (Sharma and Stevermer, 2009). Therefore, longer clinical studies with red clover would be necessary to evaluate the efficacy as a treatment of osteoporosis. Until more rigorous clinical trials are completed, soy foods and/or soy or red clover supplements cannot be recommended as alternatives for antiosteoporotic medications (Messina et al., 2004; Nieves, 2013; Poluzzi et al., 2014).

2. Others. The evidence supporting the use of other botanicals for prevention of osteoporosis is very limited. Maca prevents bone loss in rat models of estrogen-deficient bone loss (Zhang et al., 2006). Wild yam and fenugreek have effects on bone mineral density in ovariectomized rats (Chiang et al., 2011; Folwarczna et al., 2014). Silymarin has also been studied as remedy against osteoporosis in animal models (Milic et al., 2013). Horny goat weed has a long history in treating osteoporosis in traditional Chinese medicine, which was recently reviewed (Ma et al., 2011; Indran et al., 2016).

\section{Botanicals for Breast Cancer Prevention}

Many botanicals that are used for women's health are likely to have additional health beneficial effects, including antioxidant, antimicrobial, anti-inflammatory, and chemopreventive activities (Rice-Evans, 2001; Rahman et al., 2006). Although describing all these effects is beyond the scope of this review, the prevention of estrogen-dependent cancers, especially breast cancer, is summarized below for some representative women's health botanicals (Snelten et al., 2012; Dietz and Bolton, 2013; Cojocneanu Petric et al., 2015). Breast cancer prevention is especially important for postmenopausal women, because breast cancer risk increases with age and therefore postmenopausal women have a higher risk than premenopausal 
women (Wang et al., 2015b). Breast cancer is the most frequent cancer occurring in women, and it is estimated that 246,660 women will be diagnosed with breast cancer in the US in 2016 (Siegel et al., 2016). For years, researchers have studied the causes of this disease and have found a pivotal role for estrogens in the development of breast cancer (Huang et al., 2015; Ziegler et al., 2015). Four major mechanisms contribute to the overall development of estrogen-dependent cancers including hormonal, chemical, inflammatory, and epigenetic (Fig. 33).

\section{A. Hormonal Pathway}

Estrogens bind to the estrogen receptor (ER), increasing cell proliferation which leads to an enhanced risk of mutations (Fig. 23) (Santen et al., 2015). These complex processes involve genomic and nongenomic ER signaling (Bjornstrom and Sjoberg, 2005). For this review, the emphasis will be put on direct binding of phytoestrogens or estrogens to the two major estrogen receptors that are found in the normal breast tissue, $\mathrm{ER} \alpha$ and $\mathrm{ER} \beta$ (Huang et al., 2015), and their binding to the ERE (Fig. 23). Although the exact roles of the two ERs are still under intensive investigations, it has been shown that $\mathrm{ER} \alpha$ leads to proliferation of breast cells and that $\mathrm{ER} \beta$ represses proliferation and is proapoptotic (Huang et al., 2015). In postmenopausal women, estradiol is produced locally by aromatization of androgens in extragonadal sites, such as breast adipose tissue, leading to enhanced local estrogen concentrations that can lead to increased proliferation of epithelial cells (Wang et al., 2015b) (Fig. 23). Modulation of aromatase activity and thus estrogen levels is another way through which phytochemicals are able to influence the hormonal estrogen pathway.

\section{B. Chemical Pathway}

The second estrogen carcinogenesis pathway involves estrogen metabolism by $\mathrm{P} 450$ to catechols, which are oxidized to genotoxic o-quinones (Fig. 34) (Bolton, 2011; Snelten et al., 2012; Yager, 2015). P450 1A1 and 1B1, which metabolize the 2- and 4-hydroxylation pathways, respectively, are regulated by the arylhydrocarbon receptor (AhR), which binds to the xenobiotic response element (XRE) in the nucleus (Murray et al., 2014). These P450s are also responsible for the bioactivation of polycyclic aromatic hydrocarbons to the ultimate carcinogens, including polycyclic aromatic hydrocarbon quinones (Shimada, 2006; L'Heritier et al., 2014). In contrast to enhanced 2-hydroxylation of parent estrogens, which has been associated with a reduced risk of postmenopausal breast cancer (Ziegler et al., 2015), 4hydroxylation is generally considered a genotoxic pathway that has shown significant carcinogenic properties (Bolton, 2011; Snelten et al., 2012; Cavalieri and Rogan, 2014). Potentially, estrogen quinones could be eliminated by the detoxification enzymes $\mathrm{NAD}(\mathrm{P}) \mathrm{H}$-quinone oxidoreductase
(NQO1) and glutathione-S-transferase, which are regulated through the Nrf2/Keap1/antioxidant response element (ARE) pathway (Figs. 33 and 35). However, there is no direct evidence that these quinones are substrates for the detoxification enzymes, although induction of these enzymes could be considered generally cytoprotective (Chandrasena et al., 2008). Many feedback loops exist between ER, AhR, and Nrf2 pathways (Kohle and Bock, 2007; Swedenborg and Pongratz, 2010); however, as mentioned above, ER signaling crosstalk is beyond the scope of this review.

\section{Inflammatory Pathway}

Botanicals could also counteract enhanced levels of inflammatory mediators that fuel estrogen chemical carcinogenesis in the breast (Fig. 36) (Howe, 2007; Kamel et al., 2012; Vona-Davis and Rose, 2013). Cytokine secreting macrophages promote the initiation, progression, and metastasis of cancer (Obeid et al., 2013). They upregulate both aromatase and P450 1B1 expression, leading to enhanced genotoxic 4-hydroxylation products and increased DNA damage (Kamel et al., 2012; Vona-Davis and Rose, 2013). Furthermore, inflammatory mediators (e.g., NF- $\kappa \mathrm{B}$, iNOS, COX-2, IL-1 $\beta$ ) are often overexpressed in breast cancer tissues, in particular from hormone receptor negative breast cancer, and these mediators are correlated with poor prognosis (Harris et al., 2014). Many inflammatory proteins, such as COX-2, iNOS, and IL- $1 \beta$, are regulated by the transcription factor NF- $\kappa \mathrm{B}$ (Samadi et al., 2015). Numerous botanicals have been reported to inhibit $\mathrm{NF}-\kappa \mathrm{B}$ activation and/or to inhibit certain inflammatory pathways, such as COX-2, leading to prevention of inflammatory-mediated effects (Izzi et al., 2012).

\section{Epigenetic Pathway}

Finally, recent evidence suggests that epigenetic pathways can play a role in estrogen carcinogenesis (Fig. 37) (Giacinti et al., 2006; Hervouet et al., 2013; Basse and Arock, 2015). DNA methylation catalyzed by DNA methyltransferases (DNMT), posttranslational histone modification catalyzed by histone deacetylases, histone acetylases (HATs), histone methyltransferases, and histone demethyltransferases can all be involved in the carcinogenic process. As a result, phytochemicals that could interfere with these pathways might have chemopreventive effects through epigenetic modulations (Gerhauser, 2012; 2013; Byler et al., 2014; Guo et al., 2015a). In summary, estrogen carcinogenesis involves 1) hormonal, 2) chemical, 3) inflammation, and 4) epigenetic pathways (Fig. 33). Many botanicals that are used for postmenopausal women's health have been described to interfere with all or most of these four pathways, and examples of botanicals with these properties are reviewed below.

\section{E. Isoflavone-containing Botanicals (Red Clover, Soy)}

Red clover (Trifolium pratense) and soy (glycine max) contain estrogenic bioactive isoflavones, genistein and 


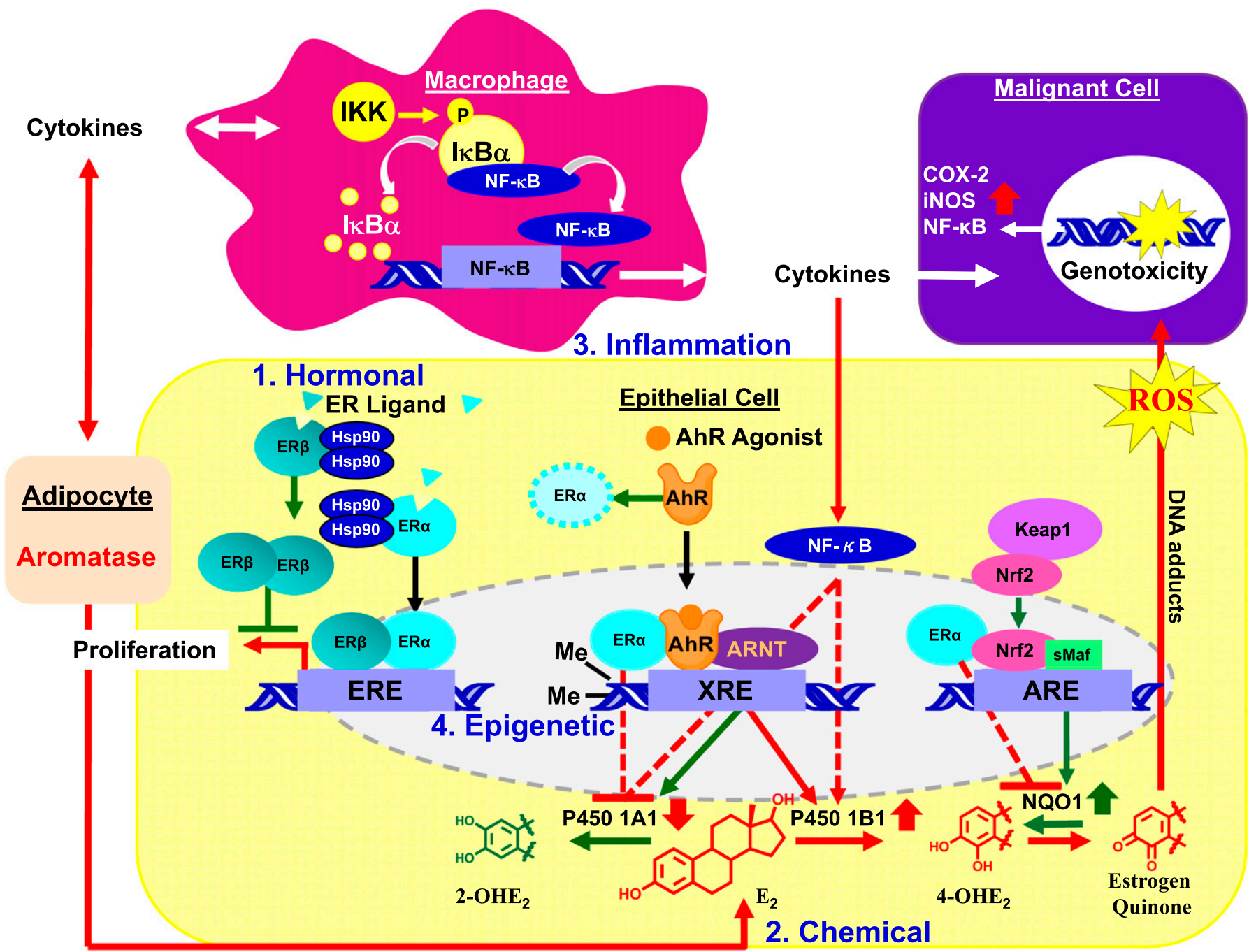

Fig. 33. Multitargeted modulation of estrogen carcinogenesis by botanicals. Estrogen carcinogenesis involves numerous pathways: (1) Hormonal (Fig. 23); estrogen binds to $\mathrm{ER} \alpha$, which activates EREs to increase proliferation. (2) Chemical (Figs. 34, 35); the aryl hydrocarbon receptor (AhR) is activated by AhR agonists, which increases P450 1A1 (benign pathway) and P450 1B1 (genotoxic pathway). Additionally, Nrf2-secondMaf binding to the antioxidant response element (ARE) increases NQO1 and phase II enzymes that detoxify genotoxic estrogen $o$-quinones, which initiate genotoxicity through DNA adducts and ROS produced from redox cycling between the catechol and estrogen-o-quinones. (3) Inflammatory (Fig. 36); inflammatory biomarkers, such as cytokines, increase aromatase expression, thus, increasing estrogen and feeding both the chemical and hormonal pathway. Cytokines, derived from chronic inflammation, macrophages or adipocytes, reportedly suppress P450 1A1, yet increase P450 1B1, most likely through the inflammatory mediator NF- $\kappa$ B. Cytokines also increase expression of genes (i.e., NF- $\kappa$ B, iNOS, COX-2) in epithelial and malignant cells that are often overexpressed in breast cancer patients. (4) Epigenetic (Fig. 37); epigenetic events through ER $\alpha$ inhibit expression of P450 1A1 by encouraging hypermethylation of its promoter, whereas having no significant effect on P450 1B1 expression. Green arrows represent potential positive effects of botanicals, whereas red arrows represent negative effects in each pathway.

daidzein as discussed in section IV.A.2.a (Fig. 24). The gut metabolite of daidzein, equol, is reported to be more estrogenic and $\operatorname{ER} \beta$ selective than daidzein (Jiang et al., 2013; van der Velpen et al., 2013). In addition to their estrogenic properties, some epidemiological data suggest that these isoflavones might contribute to the lower incidence of breast cancer in Japanese women, who eat a soy-rich diet (Adlercreutz, 2002; Yamamoto et al., 2003). Therefore, many in vitro and in vivo studies have analyzed the chemopreventive activities of genistein in particular (Russo et al., 2016). However, well-designed clinical trials analyzing the cancer preventive activity of genistein and other isoflavones or soy/red clover extracts are scarce. Isoflavones might modulate estrogen carcinogenesis through the hormonal, chemical, inflammatory, and epigenetic pathways (Dietz and Bolton, 2013; Guo et al., 2015a). Genistein in particular is a pleiotropic compound with a multitude of biological activities (Russo et al., 2016) that might modulate estrogen carcinogenesis. Whether isoflavones have breast cancer preventive properties is still a controversial topic and needs to be further analyzed in clinical trials.

1. Hormonal Pathway. In vitro studies revealed that isoflavones, such as genistein $\left(\mathrm{IC}_{50}=3.6 \mu \mathrm{M}\right)$ and biochanin $\mathrm{A}\left(\mathrm{IC}_{50}=25 \mu \mathrm{M}\right)$, are moderate inhibitors of the estrogen synthesizing enzyme aromatase (van Meeuwen et al., 2007). More potent aromatase inhibitors have been designed from the isoflavone scaffold (Bonfield et al., 2012). Genistein and daidzein (Fig. 24) have also been reported to inhibit other steroidogenic enzymes in 


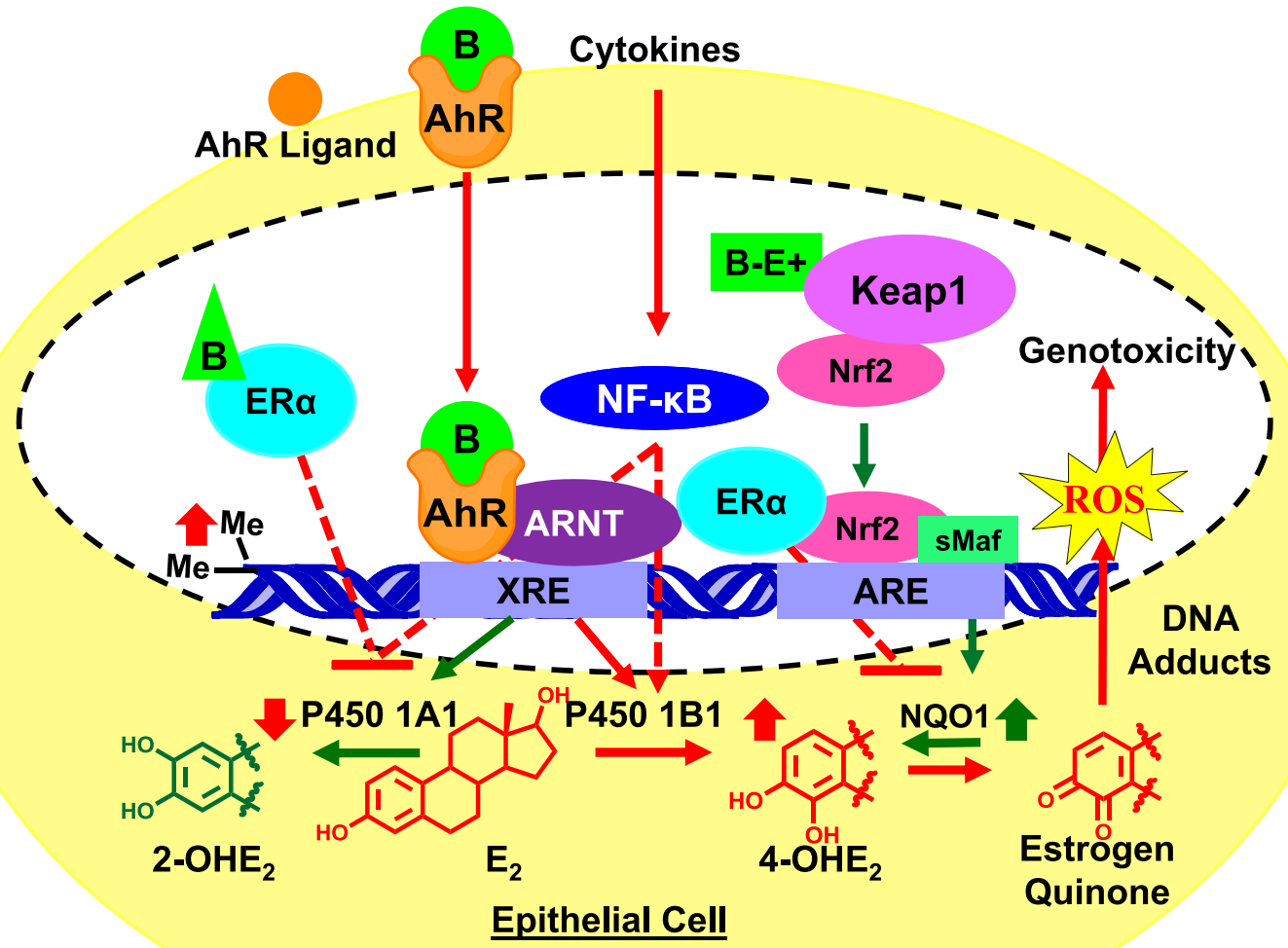

Fig. 34. Estrogen chemical carcinogenesis. AhR ligands activate XRE-responsive genes, P450 $1 \mathrm{~A} 1$ and P450 1B1, to increase benign and genotoxic estrogen metabolism, respectively. ER $\alpha$ decreases P450 1A1 expression by encouraging DNA methylation of the promoter and inflammatory cytokines that activate NF- $\kappa \mathrm{B}$ decrease P450 1A1, yet increase P450 1B1. AhR modulating, estrogenic, anti-inflammatory, and antioxidant botanicals may modulate estrogen chemical carcinogenesis by targeting AhR, ER $\alpha$, and reducing inflammatory cytokines and NF- $\kappa \mathrm{B}$ activation. Botanicals might also increase detoxification of estrogens through activating the Keap1-Nrf2-ARE pathway, thus increasing NQO1 and other detoxification enzymes.

the micromolar range, such as $3 \beta$-hydroxysteroid dehydrogenase (CYP21), which might ultimately result in lower estradiol levels (Hasegawa et al., 2013). However, other investigations describe genistein as an inducer of aromatase expression/activity in extragonadal cells, which might lead to enhanced local estrogen concentrations (Edmunds et al., 2005; Ye et al., 2009a; van Duursen et al., 2013). Genistein, daidzein, and $S$-equol are preferential ER $\beta$ agonists with nanomolar potency, but at higher concentrations, they have $\mathrm{ER} \alpha$ activities (Jiang et al., 2013). Because $\mathrm{ER} \beta$ reduces $\mathrm{ER} \alpha$-induced cell proliferation, the safety of these isoflavones likely depends on their concentrations. In the micromolar range, genistein is a selective protein tyrosine kinase inhibitor, which might lead to reduction of cell growth through inhibition of core signaling tyrosine kinases, such as epidermal growth factor receptor, insulin receptor, and others (Russo et al., 2016). For example, in an ER $\beta$-positive breast cancer cell line, genistein inhibited human epidermal growth factor 2 (HER2) overexpression and HER2 phosphorylation activity, likely through inhibition of its tyrosine kinase activity at concentrations $>1 \mu \mathrm{M}$ (Sakla et al., 2007; Russo et al., 2016). A recent study illustrated the distinct proliferative effects of genistein on different breast cancer cell types (Pons et al., 2016). In cell lines mainly expressing
$\mathrm{ER} \alpha$, genistein lead to cell proliferation, whereas in $\mathrm{ER} \beta$ expressing cells, genistein treatment had no effect. These studies illustrate that genistein has different effects on the hormonal pathway depending on concentration, ER status, and breast cell type. This might explain some of the conflicting results obtained in breast cancer cells or in animal experiments studying estrogen sensitive tissues.

Several animal and clinical studies analyzed the effect of isoflavones on endometrial and mammary tissue with controversial outcomes (Dietz and Bolton, 2013). Some in vivo experiments suggest that genistein suppresses mammary carcinogenesis (Constantinou et al., 2001; Sahin et al., 2011); however, many recent in vivo investigations found that it promotes carcinogenesis in breast tissue and enhances metastasis formation (Ju et al., 2006; Yang et al., 2015). Three animal studies demonstrated that genistein stimulates mammary gland growth and enhances the growth of breast cancer cells (MCF-7) in animals (Hsieh et al., 1998; Allred et al., 2004; Ju et al., 2006). These reports show that isoflavones of red clover/soy have the potential to reduce and/or enhance the hormonal-related carcinogenic effects of endogenous estrogens. However, the activity of the whole red clover extract or soy supplementation might be distinct from the activity of the 


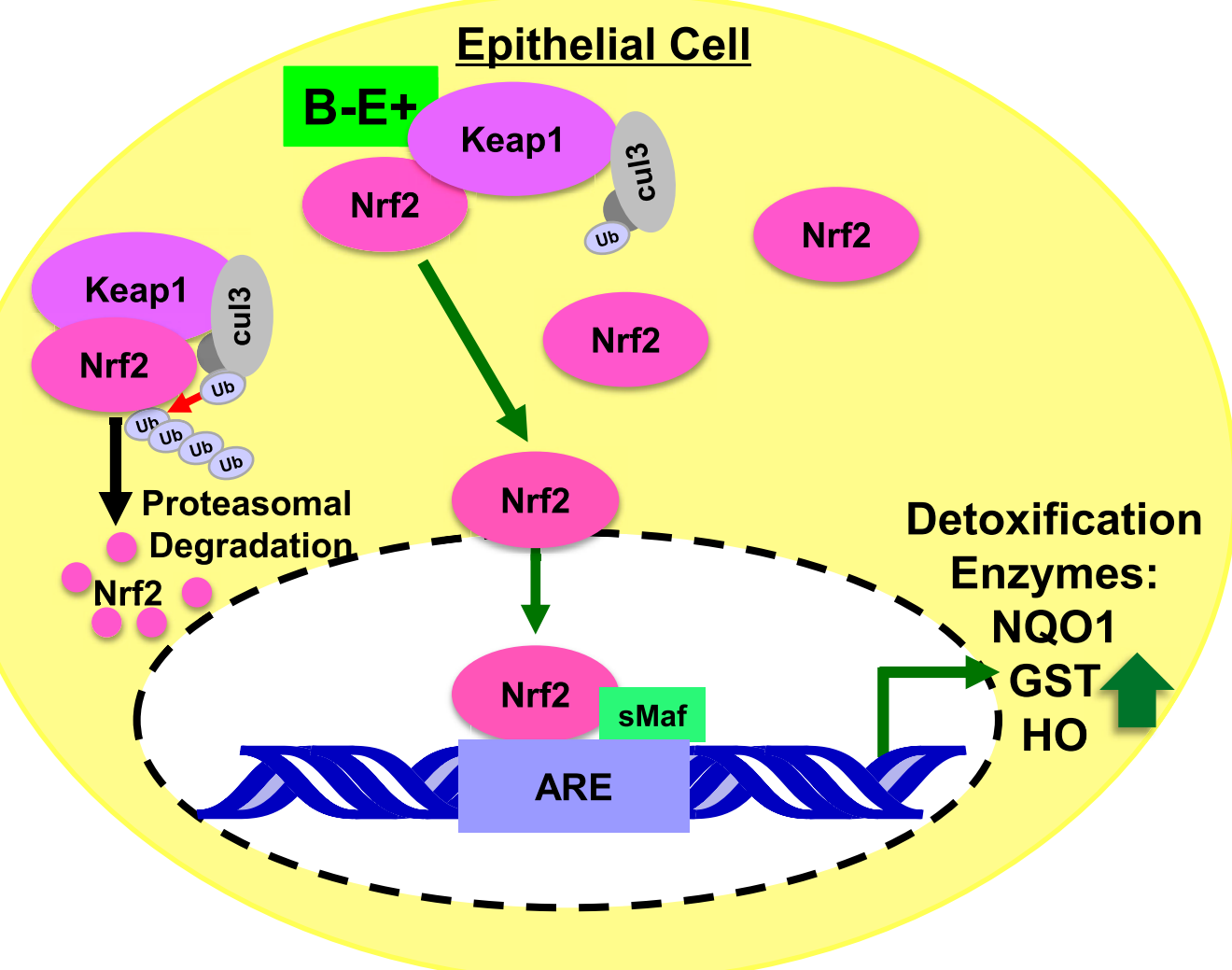

Fig. 35. Nrf2 pathway. Under normal conditions Nrf2 is associated with Keap1 in the cytosol and ubiquitinated by Cul3, causing Nrf2 proteasomal degradation. Electrophilic botanicals covalently modify cysteines in Keap1, leading to a conformational change; inhibition of Cul3-mediated Nrf2 ubiquitination, accumulation, and nuclear translocation of Nrf2; and upregulation of ARE-regulated detoxification enzymes.

isolated isoflavones. For example, uterotrophic activities have been described for genistein (Diel et al., 2004a) but not for the red clover extract (Overk et al., 2008).

Most clinical trials suggest that isoflavones in dietary supplements are safe and do not show estrogenic effects on breast or endometrial tissues. For example, in one clinical trial that used a standardized ( $26 \mathrm{mg}$ biochanin A, $16 \mathrm{mg}$ formononetin, $1 \mathrm{mg}$ genistein, and $0.5 \mathrm{mg}$ daidzein) red clover extract in women (age 49-65 years), the extract did not increase breast density over 1 year (Atkinson et al., 2004). A clinical study (up to 3 years) with postmenopausal women suggested that purified genistein ( $54 \mathrm{mg} /$ day) is safe with respect to breast and endometrial proliferation (Taylor et al., 2009). In contrast to the placebo group, the genistein arm showed a statistically significant decrease in endometrial thickness at the 36-month follow up. Genistein also significantly decreased sister chromatid exchanges in peripheral blood lymphocytes isolated from postmenopausal study participants after administration of $54 \mathrm{mg} /$ day genistein for 1 year in a randomized placebo-controlled study (Atteritano et al., 2008). Soy isoflavone dietary intervention studies with premenopausal women showed no toxicity over a period of 2 years with no effect on breast density as analyzed by mammography (Taylor et al., 2009). Similarly, a randomized, double-blind, placebo-controlled study revealed that consumption of $250 \mathrm{mg}$ of standardized soy extract
(100 mg/day isoflavones) by postmenopausal women for 10 months did not influence breast density (Delmanto et al., 2013). The correlation of plasma genistein and daidzein concentrations with breast cancer or benign breast conditions was analyzed in a breast self-examination trial in Chinese women. The subjects included women diagnosed with breast cancer or benign breast conditions and agematched controls with no known breast disease (Lampe et al., 2007). The results revealed that plasma daidzein and genistein concentrations were significantly higher in controls than in patients with breast cancer or benign breast conditions. These data may indicate selective uptake of the phytoestrogens by ER-positive tumors; however the overall chemopreventive effect of isoflavones on breast cancer is unknown.

However, some clinical studies depict an estrogenic or proliferative effect after consumption of isoflavonecontaining dietary supplements. For example, a 2 -week dietary supplementation study with soy (45 mg/day) in premenopausal women revealed an estrogenic effect as measured by modulation of estrogen-sensitive genes in nipple aspirate fluid (Taylor et al., 2009). A similar study with soy protein supplementation in premenopausal women suggested an estrogenic effect of soy due to an increase in proliferation of breast epithelium and an enhanced expression of progesterone receptors (Taylor et al., 2009). 


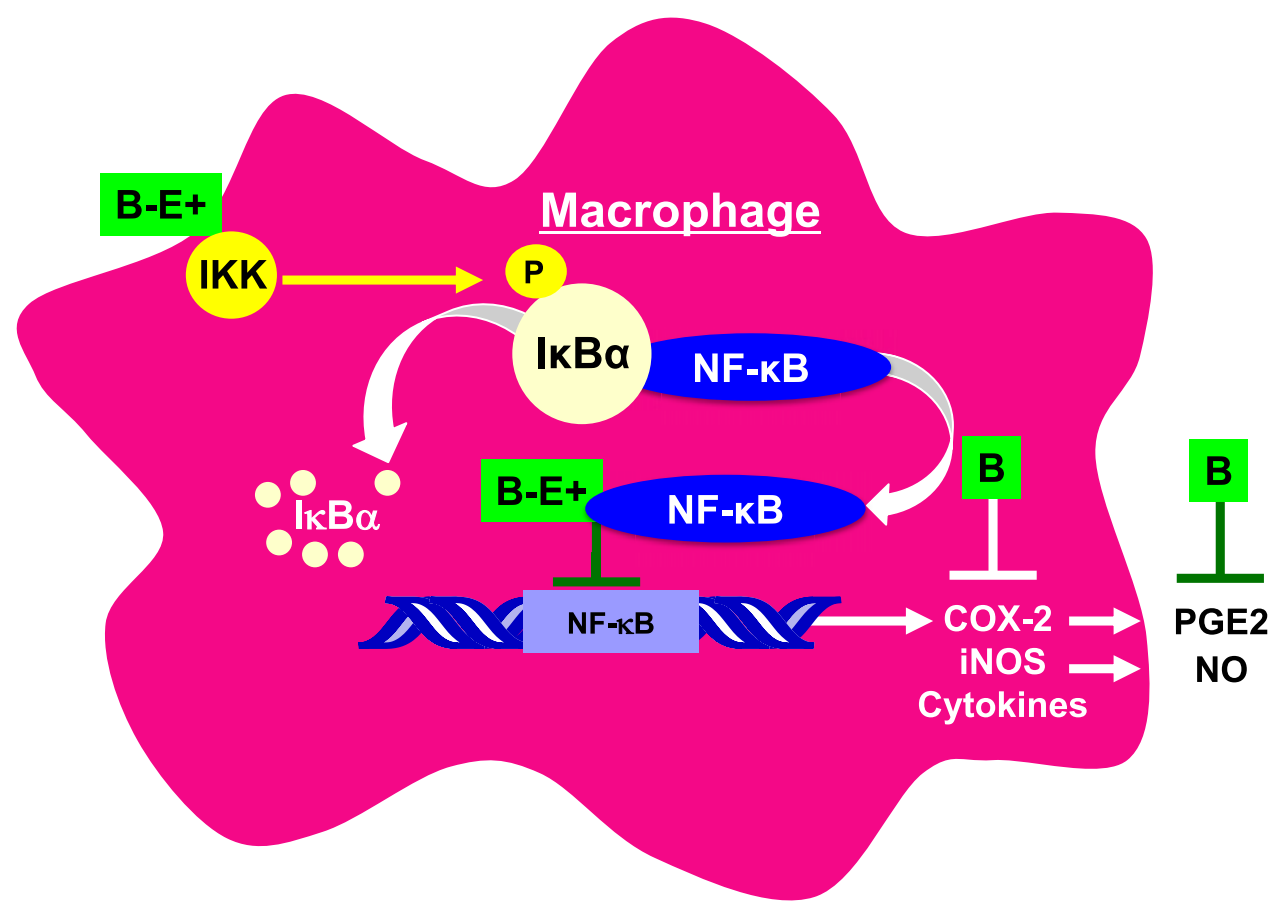

Fig. 36. Inflammatory pathway. Many botanicals are anti-inflammatory, inhibiting production of inflammatory biomarkers that are often overexpressed in breast cancer (i.e., cytokines, iNOS and COX-2). Electrophilic botanicals covalently modify both IKK and NF- $\kappa$ B to inhibit phosphorylation and DNA binding, respectively. Many phytochemicals also inhibit COX-2 and thus the production of PGE2.

A 6-month intervention study using a preparation of mixed soy isoflavones ( $150 \mathrm{mg}$ genistein, $74 \mathrm{mg}$ daidzein) was performed in healthy, high-risk adult Western women to examine the effect of these isoflavones on breast epithelial proliferation and other biomarkers in the breast tissue (Khan et al., 2012b). The study demonstrated that the isoflavones did not reduce breast epithelial proliferation, suggesting a lack of efficacy for breast cancer prevention. However, among treated premenopausal women, there was a statistically significant increase in the postintervention Ki-67 labeling index after soy supplementation. Gene expression studies demonstrated a mixed pattern, with more genes enhanced in the isoflavone supplementation group that suggested estrogenic activity. Further clinical studies with different concentrations are warranted to obtain a better picture of the hormonal and proliferative activities of isoflavone containing dietary supplements on breast tissue.

2. Chemical Pathway. Isoflavones have been shown to modulate different estrogen metabolism enzymes through a variety of pathways in in vitro and in vivo studies (Mense et al., 2008; Snelten et al., 2012). They modulate the P450 enzymes, P450 1A1 and P450 1B1, through inhibition of enzyme activity and through induction or suppression of their transcription (Wagner et al., 2008; Takemura et al., 2013). Genistein has been demonstrated to decrease P450 1A1/1B1 activity in part through direct inhibition of the enzyme in several assays in the low micromolar range (Roberts et al., 2004; Mense et al., 2008; Scott et al., 2008; Takemura et al., 2013).
Similarly, daidzein and equol have been reported to reduce $\mathrm{P} 4501 \mathrm{~A} 1$ and $1 \mathrm{~B} 1$ activity in different cell assays (Roberts et al., 2004; Scott et al., 2008).

CYP1B1 and CYP1A1 are both regulated through the AhR (Fig. 34) (Murray et al., 2014). Two AhR transactivation assays revealed biochanin A and formononetin as good AhR ligands ( $\mathrm{MM}-\mu \mathrm{M}$ range), whereas genistein, daidzein, and equol had no activity (Medjakovic and Jungbauer, 2008; Bialesova et al., 2015). Biochanin A was confirmed as

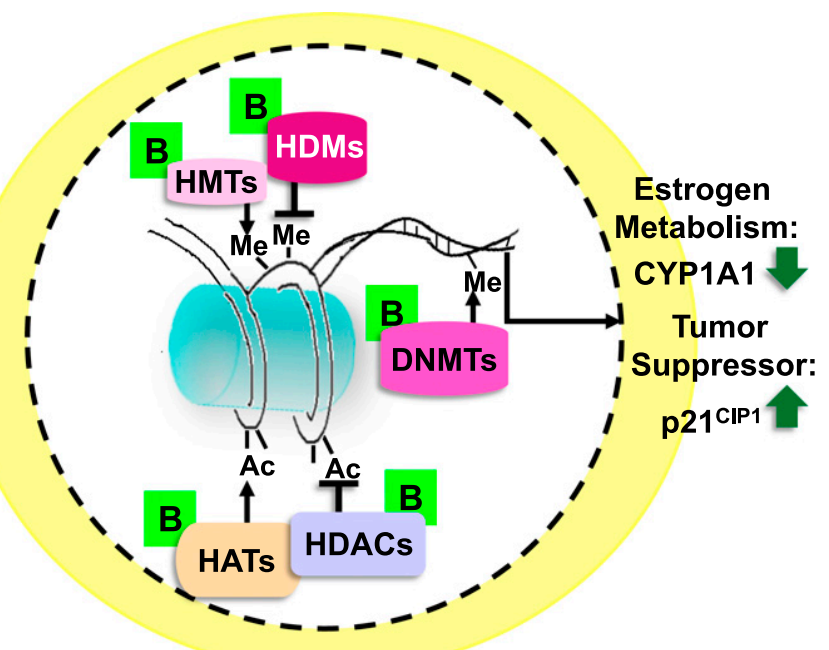

Fig. 37. Epigenetic pathway. Simplified overview of botanicals modulating epigenetic enzymes, resulting in modulation of estrogen metabolizing enzymes and tumor suppressors. HMT, histone methyltransferases; HDM, histone demethyltransferases; HAT, histone acetyltransferases; HDAC, histone deacetylases; DNMT, DNA methyltransferases. 
an AhR ligand in a TCDD competitive AhR binding assay (Han et al., 2006). Biochanin A also induced the xenobiotic response element (XRE) activity in the breast cancer cell line MCF-7 (Chan et al., 2003), and at higher concentrations (10-50 $\mu \mathrm{M})$, it caused a significant induction of CYP1A1 mRNA expression and CYP1 activity (Han et al., 2006), suggesting that biochanin A can increase oxidative estrogen metabolism. In the presence of a strong AhR agonist DMBA, biochanin A $(\geq 5 \mu \mathrm{M})$ inhibited DMBA-induced XRE activity, CYP1A1 and CYP1B1 mRNA expression, as well as DMBA-induced P450 1 EROD activity in MCF-7 cells suggesting that biochanin A can act as an AhR agonist (Chan et al., 2003; Han et al., 2006).

Interestingly, genistein and daidzein reduced CYP1A1 mRNA levels and P450 1 activity at low concentrations in MCF-7 cells (Wagner et al., 2008). Because estradiol has been reported to downregulate CYP1A1 (L'Heritier et al., 2014), the effect of genistein and daidzein on CYP1A1 might be explained by their estrogenic effects. At the tested concentrations, CYP1B1 was not influenced by genistein and daidzein. Higher genistein concentrations (5-25 $\mu \mathrm{M})$ were shown by Wei et al. (2015) to induce CYP1B1 mRNA levels. Genistein also inhibited DMBAinduced XRE-luciferase activity, P450 1 activity, CYP1A1 and 1B1 mRNA expression, and DNA adducts in the micromolar range in MCF-7 and MCF-10A cells (Chan and Leung, 2003; Leung et al., 2009). Only a limited number of in vivo studies have analyzed the influence of isoflavones on estrogen metabolism enzymes with different outcomes. One study analyzing the influence of the mixture of isoflavones on CYP1B1 and CYP1A1 in primate mammary glands and liver tissue did not show any significant effect (Scott et al., 2008). However, significant induction of CYP1A1 was observed after treatment of male Wistar rats with a standardized soybean extract containing $37 \%$ isoflavones $(100 \mathrm{mg} / \mathrm{kg})$ for 10 days (Mrozikiewicz et al., 2010).

Isoflavones have also been shown to modulate detoxification enzymes that facilitate the elimination of estradiol and its metabolites (Fig. 35) (Dietz and Bolton, 2013). Experiments in human colon cancer cells revealed that genistein $(\geq 0.1 \mu \mathrm{M})$ caused significant induction of NQO1 activity, whereas biochanin A was less effective and daidzein and formononetin were inactive (Wang et al., 1998). Genistein induced NQO1 in various cell lines as well as in different tissues in animal studies (Wang et al., 1998; Bianco et al., 2005; Froyen et al., 2009; Wiegand et al., 2009). Mechanism of action studies in ER-positive cell lines demonstrated that genistein might influence NQO1 through ER-dependent and -independent pathways (Bianco et al., 2005; Wagner et al., 2008; Kaspar and Jaiswal, 2010). It has been shown that genistein $(1 \mu \mathrm{M})$ and daidzein $(10 \mu \mathrm{M})$ reduced NQO1 mRNA levels and catechol-O-methyltransferase mRNA and activity similar to estradiol in MCF-7 cells likely through an $\mathrm{ER} \alpha$-Nrf2-mediated mechanism
(Wagner et al., 2008). However, in ER $\beta$-positive cells, the isoflavones upregulated NQO1 mRNA through an ER $\beta$-Nrf2-mediated mechanism (Bianco et al., 2005; Froyen and Steinberg, 2011). These variable results suggest that the NQO1 induction potential of genistein may be dependent on the cell type and ER status.

The influence of isoflavones on other detoxification enzymes might be less pronounced. Although genistein induced hepatic NQO1 activity, it did not induce the activity of other detoxification enzymes (glutathione- $S$ transferase, catalase, glutathione peroxidase, superoxide dismutase) (Wiegand et al., 2009). However, in a topical inflammation model, in which 1 or $2 \mu \mathrm{g}$ of a soy isoflavone product was administered, the activity of the detoxification enzymes catalase and superoxide dismutase were induced, as well as glutathione levels (Khan et al., 2012a). An in vivo study in Sprague-Dawley rats (7 days) analyzed the effect of biochanin A on the Phase II enzyme sulfotransferase that is involved in the metabolism of estradiol and detoxification of various carcinogens (Raftogianis et al., 2000; Chen et al., 2010b). The study revealed that biochanin $\mathrm{A}(10 \mathrm{mg} / \mathrm{kg} /$ day $)$ induced the expression and activity of sulfotransferase $1 \mathrm{~A} 1$ and $2 \mathrm{~A} 1$ in female rat liver and intestine.

In conclusion, the isoflavones have the potential to modulate estrogen metabolism. Biochanin A and formononetin seem to be $\mathrm{AhR}$ agonists and have the highest potential to induce P450 1A1/1B1 (Han et al., 2006; Bialesova et al., 2015). However, in vivo, biochanin A and formononetin are rapidly metabolized to genistein and daidzein, respectively (Fig. 24), and their concentration is likely relatively low (Piersen et al., 2004). Soy supplements contain much less biochanin A and formononetin than red clover extracts (Burdette and Marcus, 2013) and might therefore have a better safety profile with respect to estrogen metabolism. In general, the influence of genistein on the chemical pathway is probably cell and ER-status dependent. Therefore, more in vivo studies are warranted to analyze whether soy and red clover would reduce or enhance genotoxic estrogen metabolism.

3. Inflammatory Pathway. Anti-inflammatory activities have been described for soy, red clover, and their isoflavones in in vitro and in vivo studies (Banerjee et al., 2008; Seibel et al., 2009; Masilamani et al., 2012; Nagaraju et al., 2013; Ganai et al., 2015). For example, a red clover extract (40\% isoflavones) and isolated isoflavones reduced the proinflammatory cytokines IL-6 and TNF- $\alpha$ in lipopolycaccharide (LPS)-induced macrophages (Mueller et al., 2010). Biochanin A exhibited the strongest TNF- $\alpha$ inhibition $\left(\mathrm{IC}_{50}=6 \mathrm{nM}\right)$, followed by genistein $\left(\mathrm{IC}_{50}=40 \mathrm{nM}\right)$, whereas IL- 6 was most efficiently inhibited by genistein and equol $\left(\mathrm{IC}_{50}=8\right.$ and $6 \mathrm{nM}$, respectively). Other studies reported the reduction of inflammatory cytokines by genistein, such as IL-4, IL-5, interferon- $\gamma$, in a murine model of asthma (Gao et al., 2012). 
Various studies demonstrated the ability of isoflavones to reduce NF- $\kappa \mathrm{B}$ pathways in different cell lines in the micromolar range (Gong et al., 2003; Kole et al., 2011; Masilamani et al., 2012; Wu et al., 2015b). Further analysis revealed that one mechanism through which genistein might reduce nuclear translocation of NF- $\kappa \mathrm{B}$ is likely the inhibition of kinase pathways leading to a reduced phosphorylation of the NF- $\kappa \mathrm{B}$ inhibitory protein $\mathrm{I} \kappa \mathrm{B} \alpha$ (Fig. 36) (Gong et al., 2003; Masilamani et al., 2012; Ganai et al., 2015) or to a decreased phosphorylation of the NF- $\kappa$ B subunit p65 (Chung et al., 2014). Similarly, biochanin A has been shown to inhibit LPS-induced NO, IL-6, IL- $1 \beta$, and TNF- $\alpha$ production in macrophages through prevention of phosphorylation and degradation of $\mathrm{I} \kappa \mathrm{B} \alpha$ (Kole et al., 2011). In certain cell lines, a role for the ER in inhibiting NF- $\kappa \mathrm{B}$ pathways has been postulated (Sas et al., 2012) and that isoflavones, such as genistein, might reduce inflammatory responses through their ER binding activity (Dijsselbloem et al., 2004).

Various in vitro and in vivo studies demonstrated that isoflavones repress or inhibit COX-2 activity in the micromolar range (Lam et al., 2004; Horia and Watkins, 2007; Seibel et al., 2009; Mueller et al., 2010; Chung et al., 2014). Comparison of the COX-2 inhibiting potential of the four isoflavones (Fig. 24) in murine macrophages showed genistein had the highest activity at $1 \mu \mathrm{M}$ compared with $10 \mu \mathrm{M}$ for biochanin $\mathrm{A}$ and $40 \mu \mathrm{M}$ for formononetin and daidzein (Lam et al., 2004). Genistein $(1 \mu \mathrm{M})$ also significantly inhibited 12-O-tetradecanoylphorbol-13-acetate (TPA)-induced prostaglandin E2 (PGE2) synthesis in ER negative breast cancer cells (MDA-MB-231) (Horia and Watkins, 2007). In addition, it significantly reduced the invasion capacity of this cell line at $10 \mu \mathrm{M}$, suggesting a chemopreventive potential of genistein in ER negative breast cancer cells. Pretreatment of human nontumorigenic breast epithelial cells $(\mathrm{MCF}-10 \mathrm{~A})$ with genistein $(\approx 46 \mu \mathrm{M})$ reduced TPA-induced COX-2 expression and PGE2 synthesis through reduction of TPA-induced transcriptional activity of NF- $\kappa \mathrm{B}$ (Chung et al., 2014). Genistein also reduced COX-2 expression in animal models of inflammation (Hwang et al., 2009; Willenberg et al., 2015). Because COX-2 is reported to be upregulated in breast cancer and epidemiological studies suggest a protective effect of COX inhibitory drugs on breast cancer (Howe, 2007), further in vivo studies are needed to determine if long-term treatment with isoflavone containing dietary supplements might modulate COX-2 in the breast tissue.

In a 2-year soy intervention study, 183 premenopausal women consumed either two daily soy servings containing $50 \mathrm{mg}$ of isoflavones (aglycone equivalents) or their regular diet (Maskarinec et al., 2009). Serum samples were obtained in regular intervals and were analyzed for the inflammatory markers IL-6, C-reactive protein, leptin, and adiponectin by enzyme-linked immunosorbent assay. No significant effect of the soy food was determined; however, the leptin value increased in the control but not in the soy group. In a single-blind, randomized, controlled trial, 31 postmenopausal women received either three servings of soy milk or reduced fat dairy milk for 4 weeks (Beavers et al., 2009). Plasma markers of inflammation and oxidative stress (TNF- $\alpha$, IL-6, IL- $1 \beta$, COX-2, superoxide dismutase, and glutathione peroxidase) were determined before and after supplementation; however, no significant effects of the soy milk intervention were observed for any of these markers.

In conclusion, although there are clear antiinflammatory activities reported for isoflavones from in vitro studies, the in vivo experiments and clinical trials analyzing the inflammatory pathways are less conclusive (Beavers et al., 2009; Maskarinec et al., 2009; Willenberg et al., 2015). More clinical trials will be necessary to evaluate the anti-inflammatory potential of isoflavones, soy, and red clover dietary supplements for their potential clinical application.

4. Epigenetic Pathway. Recent studies indicated that isoflavones might exert cancer chemopreventive effects by targeting epigenetic mechanisms (Guo et al., 2015a; Hwang and Choi, 2015). Various in vitro studies demonstrated that genistein suppresses DNA methylation in breast cancer cells, possibly leading to increased mRNA expression of tumor suppressor genes (Guo et al., 2015a). For example, genistein (Fig. 24) has been shown to decrease mRNA levels and protein expression of DNA methyltransferase 1 (DNMT) 1 in MCF-7 cells. DNMT 1 has been reported to be upregulated in breast cancer tissue (Mirza et al., 2013). Also, genistein, has been reported to reactivate $\mathrm{ER} \alpha$ expression and resensitize $\mathrm{ER} \alpha$-dependent cellular responses to $\mathrm{E}_{2}$ in $\mathrm{ER} \alpha$-negative breast cancer cells, MDA-MB 231, in the micromolar range through epigenetic effects ( $\mathrm{Li}$ et al., 2013c). In addition, genistein and daidzein in the micromolar range decreased hypermethylation of the oncosuppressor genes, tumor suppressor gene 1 and tumor suppressor gene 2, resulting in restored activity of these genes in different breast cell lines (Bosviel et al., 2012). Treatment with a genistein-enriched diet $(250 \mathrm{mg} / \mathrm{kg}$ genistein) inhibited breast cancer development in a breast cancer xenograft model perhaps due to epigenetic regulation of the tumor suppressor genes p21 and p16 (Li et al., 2013a).

Isoflavones might have protective effects toward breast carcinogenesis when a diet rich in soy products is consumed early in life (Warri et al., 2008). This is in part based on the protective effect of a lifelong high-soy diet on breast cancer in Asian women. On the basis of this information, an in vivo study analyzed the influence of lifelong exposure to an isoflavone-rich diet on mammary gland proliferation, other estrogen sensitive endpoints, and on epigenetic markers (Blei et al., 2015). The authors concluded that isoflavones might alter the morphology of the mammary gland during pubertal breast growth and epigenetic modulation might play an important role. These effects might ultimately have 
an influence on the likelihood of breast carcinogenesis later in life; however, further studies are necessary to support this theory. In general, epidemiological studies suggest that soy consumption is associated with less breast cancer risk in Japanese women with an enriched soy diet (Yamamoto et al., 2003); however, the correlation between isoflavone intake and breast cancer risk is less clear in American women. The association between dietary isoflavone intake with breast cancer risk was analyzed using population-based, observational data from the Multiethnic Cohort Study (MEC) in 84,450 mostly postmenopausal American women, who were categorized based on their isoflavone intake (highest consumed amount: $20.3-178.7 \mathrm{mg} /$ day) (Morimoto et al., 2014). After a mean follow up of 13 years, no statistical association between dietary isoflavone intake and overall breast cancer risk was observed. This study is in agreement with other epidemiological prospective studies suggesting that high soy intake in Asian women (around 25 and $50 \mathrm{mg}$ of isoflavones) starting earlier in life shows a significant trend of breast cancer risk reduction; however, studies with Western women consuming a low soy diet do not show a significant association between dietary isoflavone exposure and breast cancer risk (Wu et al., 2008a; Dong and Qin, 2011).

In conclusion, numerous studies have shown that isoflavones, in particular genistein, might modulate estrogen carcinogenesis through hormonal, chemical, inflammatory, and epigenetic pathways. The data analyzing the influence of isoflavones on cell proliferation (hormonal) and on estrogen metabolism enzymes (chemical) reveal conflicting results, suggesting that isoflavones might enhance or decrease estrogen carcinogenesis through both hormonal and chemical pathways. The concentrations and cell type may play an important role whether isoflavones reduce or increase estrogen carcinogenesis. There is clear evidence mostly from in vitro studies that isoflavones, in particular genistein, have anti-inflammatory properties that might decrease estrogen carcinogenesis in the breast. However, dietary in vivo studies show conflicting results and it is not clear whether the bioavailability of genistein and of the other isoflavones in dietary supplements are sufficient to exert significant clinical anti-inflammatory activity. Epigenetic effects with subsequent reactivation of tumor suppressor genes and other genes have been shown (e.g., genistein). Some in vivo studies propose that lifelong exposure to isoflavones might modulate mammary gland development during puberty, potentially leading to breast cancer prevention. Overall, clinical studies often indicate no breast cancer preventive effect with low isoflavone intake in the diet during adulthood in Western cultures. However, some studies show a protective effect when isoflavones are enriched in the diet throughout life as in the case of Asian populations. Future animal and clinical studies are needed to determine if isoflavones are safe and whether they have the ability to elicit chemopreventive properties at clinically relevant concentrations.

\section{$F$. Humulus lupulus (Hops)}

In addition to the estrogenic properties of hops, which are mediated by the potent $\mathrm{ER} \alpha$ phytoestrogen 8-PN (Fig. 26) (section IV.A.2.a), chemopreventive properties have been described for hop extracts and especially for the major prenylated polyphenol, the chalcone xanthohumol (XH) (Fig. 26) (Chadwick et al., 2004; Plazar et al., 2007). XH is an electrophilic compound that exerts multiple chemopreventive effects at least in part through binding to sulfhydryl groups in proteins, such as Keap1 (Liu et al., 2005), leading to the induction of the detoxification enzyme NQO1 (Fig. 35) (Dietz et al., 2005a, 2013). In addition, XH has been shown to induce apoptosis (Strathmann et al., 2010) and to exhibit anti-inflammatory activities (Harikumar et al., 2009; Dorn et al., 2013). Some in vitro and in vivo studies suggest that hop extracts might influence the hormonal, chemical, and inflammatory pathways of estrogen carcinogenesis. Data on the potential influence of hops and its bioactive compounds on the epigenetic pathway are currently not available. Clinical investigations that analyze the chemopreventive properties in human are also lacking.

1. Hormonal Pathway. As outlined in section IV. A.2.b, 8-PN (Fig. 26) is a potent nanomolar $\mathrm{ER} \alpha$ agonist (Overk et al., 2005; Helle et al., 2014). Various in vitro and in vivo studies demonstrate proliferative activities on breast cells/tissue or uterotrophic activities (Diel et al., 2004b; Overk et al., 2008). These proliferative activities might enhance hormonal estrogen carcinogenesis and may interfere with concurrent breast cancer treatments (van Duursen et al., 2013). However, XH has been reported to have antiproliferative activities in the micromolar range in several cancer cell lines (Vanhoecke et al., 2005; Yoshimaru et al., 2014). For example, in ER $\alpha$-positive cells, $\mathrm{XH}$ suppressed $\mathrm{E}_{2}$ signaling through reactivation of the tumor suppressor protein prohibitin 2 (Yoshimaru et al., 2014). XH has also been reported to inhibit cell invasion through upregulation of the epithelial cadherin/ catenin complex in MCF-7 cells (Vanhoecke et al., 2005). Because the concentration of XH in hops extracts is usually much higher than that of 8-PN (van Breemen et al., 2014), $\mathrm{XH}$ might reduce the proliferative activities of 8-PN in the whole hop extract. For example, a hops extract showed estrogenic activities in vitro; however, in vivo studies did not reveal uterotrophic activities, suggesting that either the bioavailable 8-PN concentrations were not high enough or that the extract may contain various counteracting phytoconstituents such as XH or phytoprogestins (Overk et al., 2008). In general, 8-PN is a minor compound in hops, but it can accumulate in vivo through metabolism of IX by P450s or intestinal bacteria (Fig. 26) (Possemiers et al., 2006; van Breemen et al., 2014). The overall estrogenic properties are therefore highly dependent on the composition of the hops 
extract and the individual metabolism and duration of treatment in vivo.

Aromatase inhibition has also been reported for 8-PN $(300 \mathrm{nM})$ in microsomes of human placental tissue or fibroblasts from healthy human mammary tissue (van Meeuwen et al., 2008). Similarly, in a human adrenal gland cell line, H295R, 8-PN was a potent aromatase inhibitor with an $\mathrm{IC}_{50}$ of $0.1 \mu \mathrm{M}$ (van Duursen et al., 2013). Interestingly, coculture of H295R cells with MCF-7 cells led to decreased estradiol concentrations and predominantly to an increased progesterone and $17 \alpha-\mathrm{OH}$-progesterone level. In breast cancer cells, 8-PN showed the highest aromatase inhibiting activity compared with IX and $\mathrm{XH}$ with an $\mathrm{IC}_{50}$ of $0.08 \mu \mathrm{M}$ (Monteiro et al., 2007). XH inhibited aromatase with a lower $\mathrm{IC}_{50}$ of $3.2 \mu \mathrm{M}$, and IX showed the weakest activity $\left(\mathrm{IC}_{50}=25.4 \mu \mathrm{M}\right)$.

2. Chemical Pathway. Several hop polyphenols (Fig. 26) have been analyzed for their potential to inhibit P450 $1 \mathrm{~A} 1$ and $1 \mathrm{~B} 1$, which are involved in estrogen metabolism (Henderson et al., 2000). Several of these compounds showed significant inhibitory potential for these P450 enzymes at $10 \mu \mathrm{M}$. However, 8-PN was also able to moderately induce CYP1A2 mRNA levels (GrossSteinmeyer et al., 2009). Recently, P450 1A1 and 1B1 inhibiting potential has been confirmed for IX, 8-PN, XH, and 6-PN with $\mathrm{IC}_{50}$ values in the high nanomolar to low micromolar range (Wang et al., 2016). 8-PN and 6-PN have also been shown to be agonists for the AhR receptor, resulting in an increase in AhR-mediated XRE-luciferase activity in HepG2 and MCF-7 cells. 6-PN also gave a significant increase in CYP1A1 gene expression, P450 1 activity, and estrogen 2-hydroxylation in MCF-10A and MCF-7 cells (Fig. 34). These data suggest that hops favors induction of the nongenotoxic 2-hydroxylation pathway in breast cells (Wang et al., 2016). Another study reported that a hop extract inhibited estrogen-induced malignant cell transformation of MCF-10A cells, suggesting that hops might have chemopreventive activity potentially through attenuation of the genotoxic estrogen metabolism pathway (Hemachandra et al., 2012).

Hops and its major Michael acceptor, $\mathrm{XH}$, also influence detoxification enzymes. They have been shown to induce NQO1 activity in murine hepatoma cells, leading to prevention of menadione-induced DNA damage at low micromolar concentrations (Dietz et al., 2005a). XH has also been shown to increase the levels of Nrf2, GSTA, and GSTP in human hepatocarcinoma HepG2 cells, whereas in normal hepatocytes it increased the levels of Nrf2, GSTs, HO-1, and NQO1 in addition to p53 (Krajka-Kuzniak et al., 2013). XH also upregulated the transcription of NQO1 and HO-1 in mouse microglial cells via the Nrf2 pathway (Lee et al., 2011a). The in vivo NQO1-inducing potential by hops and XH has been confirmed in liver tissue of SpragueDawley rats (Dietz et al., 2013). These data suggest the potential of hops and its abundant Michael acceptor,
$\mathrm{XH}$, in modulating cytoprotective enzymes leading to chemoprevention.

3. Inflammatory Pathway. Various in vitro and in vivo studies describe anti-inflammatory activities for hop extracts (Saugspier et al., 2012; Starkel et al., 2015) and XH (Hougee et al., 2006; Lupinacci et al., 2009; Dorn et al., 2012). Lupinacci et al. (2009) reported that a hop extract $(0.1 \mu \mathrm{g} / \mathrm{ml})$ significantly inhibited the production of the proinflammatory chemokine monocyte chemoattractant protein-1 in LPS-activated RAW 264.7 mouse macrophages. Bioassay guided fractionation of the extract revealed $\mathrm{XH}(2.5 \mu \mathrm{g} / \mathrm{ml})$ as the major antiinflammatory compound (Lupinacci et al., 2009; Dietz and Bolton, 2013). XH (micromolar range) inhibited the activation or binding of the transcription factor NF- $\kappa \mathrm{B}$ in macrophages, glia cells, hepatic stellate cells, or breast cancer cells, thus reducing the induction of proinflammatory genes and cytokines (Harikumar et al., 2009; Cho et al., 2010; Lee et al., 2011a; Dorn et al., 2012). Studies in mouse microglial cells showed that the antiinflammatory activity of $\mathrm{XH}$ depended on the transcription factor Nrf2, in addition to NF- $\kappa \mathrm{B}$ (Lee et al., 2011a). Other mechanism of action studies showed that XH might inhibit TNF-induced $\operatorname{IkB} \alpha$ phosphorylation and degradation, likely through interacting with cysteine residues of IKK and p65, thus leading to suppression of nuclear translocation of $\mathrm{p} 65$ and inhibition of $\mathrm{NF}-\kappa \mathrm{B}$ pathways (Harikumar et al., 2009). In vivo anti-inflammatory activities have also been reported for XH (Dietz and Bolton, 2013; Gupta et al., 2014). In a chronic dermatitis model in the mouse ear, topical XH treatment reduced the degree of ear thickening induced by oxazolone, suggesting that $\mathrm{XH}$ reduces skin inflammation (Cho et al., 2010). In two animal models of liver injury and inflammation, feeding of XH reduced mRNA levels of hepatic inflammatory markers (e.g., IL-1 $\alpha$, monocyte chemoattractant protein-1) (Dorn et al., 2012 , 2013). The mechanism seemed at least in part through decrease of NF- $\kappa \mathrm{B}$ activity (Dorn et al., 2012). Interestingly, oral administration of XH $(100 \mu \mathrm{M})$ to nude mice inoculated with MCF-7 cells resulted in a significant decrease in NF- $\kappa \mathrm{B}$ activity, reduced phosphorylated $\mathrm{I} \kappa \mathrm{B} \alpha$ staining, and a decrease in the inflammatory interleukin IL- $1 \beta$, which is often increased in breast cancer (Monteiro et al., 2008). Although the tumor weights between the control and XH group were not significantly different, $\mathrm{XH}$ did significantly increase the amount of apoptotic cells and decreased microvessel density in the tumors (Monteiro et al., 2008). A spent hops extract, supplemented to the diet of 5 -week-old pigs for 4 weeks, also led to a significant decrease of the inflammation marker IL- $1 \beta$ in the duodenum, ileum, and colon (Fiesel et al., 2014).

COX-2 is another inflammatory marker in breast cancer that has been shown to be inhibited by many phytochemicals. COX-2 inhibition has been demonstrated for a hop extract and XH (Gerhäuser et al., 2002; Hougee et al., 2006). A hops $\mathrm{CO}_{2}$ extract inhibited 
PGE2 production in LPS-stimulated peripheral blood monocytes $\left(\mathrm{IC}_{50}=3.6 \mu \mathrm{g} / \mathrm{ml}\right)$ likely through inhibition of COX-2 $\left(\mathrm{IC}_{50}=20.4 \mu \mathrm{g} / \mathrm{ml}\right.$ ) (Hougee et al., 2006). Mice treated with this hop extract showed decreased production of PGE2 in a zymosan-induced acute arthritis model; however, the extract did not reduce joint swelling (Hougee et al., 2006). XH, but not IX, moderately inhibited COX-1 and COX-2 activity in microsomal fractions $\left(\mathrm{IC}_{50}=16.6 \mu \mathrm{M}\right.$ and $41.5 \mu \mathrm{M}$, respectively) (Gerhäuser et al., 2002), suggesting that other hop compounds might add to the COX inhibiting activity of hops extracts.

4. Epigenetic Pathway. The influence of hops or its compounds on epigenetic pathways has not been examined in detail. One study analyzed protein targets of the electrophile $\mathrm{XH}$ in the breast cancer cell line MCF-7/6 (Wyns et al., 2012). Immunoprecipitation experiments using a mouse-XH-antibody revealed that $\mathrm{XH}$ binds to proteins of the histone H2A family. Therefore, $\mathrm{XH}$ may exert epigenetic effects through modification of histones that could influence gene expression, potentially leading to chemopreventive effects.

In summary, the in vitro and in vivo studies suggest that hops may modulate estrogen carcinogenesis. 8-PN elicits estrogenic, proliferative, and aromatase inhibiting activities in the nanomolar range; however, other hops phytochemicals might counteract these proliferative activities. The CYP inhibiting activity of 8-PN might also influence estrogen metabolism, although much higher concentrations are needed (micromolar) for these effects, which may not be clinically relevant. 6-PN has been shown to be a potent inducer of P450 1A1, which catalyzes the benign estrogen 2-hydroxylation pathway leading to lower estrogen levels. Hops and XH have been shown to exert chemopreventive activity in vitro and in vivo through the Keap1-Nrf2 pathway, resulting in the induction of detoxification enzymes such as NQO1 and HO-1. Anti-inflammatory activities of hops and $\mathrm{XH}$ have been reported in vitro and in vivo through modulation of NF- $\kappa \mathrm{B}, \mathrm{COX}-2$, and prostaglandins. Hops and $\mathrm{XH}$ might have the potential to exhibit chemoprevention via epigenetic mechanisms, which needs further studies. Although a human pharmacokinetic study demonstrated the bioavailability of the hops compounds 8-PN and XH (van Breemen et al., 2014), clinical studies are needed to analyze the chemopreventive activities of hops. It is also important to define whether the concentrations of the bioactive compounds in standardized hop dietary supplements are sufficient to achieve chemopreventive effects in women.

\section{G. Glycyrrhiza Species (Licorice)}

As discussed in section IV.A.2.c, estrogenic properties have been reported for several licorice species and liquiritigenin (Fig. 27) is believed to be one of the estrogenic compounds (Hajirahimkhan et al., 2013b). Some studies suggest licorice could modulate proliferation in estrogen-sensitive cells/tissues, which could prevent hormonal carcinogenesis (Mersereau et al., 2008; Tang et al., 2015). Licorice also contains electrophilic compounds such as isoliquiritigenin and licochalcone A that might induce chemoprevention through activation of apoptotic pathways, anti-inflammatory effects, and inhibition of oxidative estrogen metabolism (Webb et al., 1992; Jo et al., 2004; Takahashi et al., 2004; Cuendet et al., 2006; Wu and Hsieh, 2011; Tsai et al., 2014; Dunlap et al., 2015; Peng et al., 2015; Tang et al., 2015). Involvement of the three medicinally used species of licorice (G. glabra, G. uralensis, and G. inflata) and their compounds in various mechanisms of breast cancer prevention is reviewed below.

1. Hormonal Pathway. It has been shown that extracts of Glycyrrhiza glabra and G. uralensis stimulated MCF-7 (ER $\alpha$ ) cell growth at low concentrations (Dong et al., 2007; Hu et al., 2009). In contrast, G. uralensis was reported to have antiproliferative effects in MCF-7 breast cancer cells at higher concentrations (Jo et al., 2004, 2005). The estrogenic compound liquiritigenin did not enhance proliferation of MCF-7 (ER $\alpha)$ xenograft or induce uterine weight in nude mice (Mersereau et al., 2008), likely because of its ER $\beta$ selectivity. The marker compound in G. glabra, glabridin (Fig. 27), was shown to stimulate ER-dependent cell growth at concentrations lower than $10 \mu \mathrm{M}$ and to inhibit cell growth at concentrations higher than $15 \mu \mathrm{M}$ (Tamir et al., 2000). Selective estrogen receptor modulator-like activities of isoliquiritigenin and liquiritigenin have also been reported in an array of cell based assays (Boonmuen et al., 2016). Finally, isoliquiritigenin inhibited aromatase in MCF-7 cells stably transfected with CYP19 (MCF-7aro) and deterred the growth of MCF-7aro xenograft tumors in ovariectomized athymic mice when administered in the diet (Ye et al., 2009b).

2. Chemical Pathway. Licorice species and its bioactive compounds have been shown to differentially modulate estrogen metabolism and thus may have varied effects on breast cancer prevention. It was shown that G. glabra and G. uralensis as well as isoliquiritigenin increased the genotoxic estrogen 4-hydroxylation pathway through enhanced AhR-mediated induction of P450 1B1 activity in MCF-10A cells (Dunlap et al., 2015). However, in the same study G. inflata and its specific compound licochalcone A (Fig. 27) inhibited estrogen metabolism by antagonizing AhR and blocking XRE at the promoter region of CYP1B1 (Dunlap et al., 2015). When DMBA or TCDD were used as the AhR agonists in MCF-7 cells, isoliquiritigenin reduced AhR translocation to the nucleus and diminished AhR interaction with XREs leading to downregulation of DMBA and TCDD-mediated induction of CYP1A1, 1A2, and 1B1 (Wong et al., 2014). Similarly, G. glabra extract suppressed the tumorigenic effects of TCDD by downregulating $A h R$, arylhydrocarbon receptor nuclear translocator, and CYP1A1 genes and inducing cell cycle 
arrest in MCF-7 cells (Chu et al., 2014). These data suggest that different species of licorice could have a variety of effects on estrogen metabolism, which further emphasizes the importance of authentication of material and standardization to bioactive compounds.

Various licorice species have also been shown to differentially modulate detoxification pathways. G. uralensis was reported to induce Nrf2-mediated genes in hepatoma cells and animal tissues (Wu et al., 2011). In a comparative analysis, G. glabra, G. uralensis, and G. inflata induced NQO1 dose dependently in murine hepatoma (hepa1c1c7) and breast epithelial (MCF-10A) cells (Hajirahimkhan et al., 2015). However, when G. glabra and $G$. uralensis were administered orally to female rats, no NQO1 induction was observed in liver, whereas G. glabra had a slight but significant NQO1 induction in mammary tissue (Hajirahimkhan et al., 2015). It was shown that the common Michael acceptor of licorice species, isoliquiritigenin activated NQO1 dose dependently in vitro but failed to induce this enzyme in liver and mammary glands of the female rats when administered orally (Hajirahimkhan et al., 2015). Other studies showed slight increase in NQO1 at very high doses of isoliquiritigenin, but the effect did not lead to the inhibition of mammary tumor growth (Cuendet et al., 2006, 2010). These observations with isoliquiritigenin could be associated with its conversion to the estrogenic compound liquiritigenin as discussed earlier and also to the low bioavailability of isoliquiritigenin in vivo (Hajirahimkhan et al., 2013b, 2015; Simmler et al., 2013). Licochalcone A in G. inflata is also a Michael acceptor and has been shown to induce NQO1 in hepa1c1c7 and MCF-10A cells (Kondo et al., 2007a; Hajirahimkhan et al., 2015; Simmler et al., 2015a). These data suggest that various species of licorice could differentially modulate the chemical estrogen carcinogenesis pathway.

3. Inflammatory Pathway. Licorice has been traditionally used for several ailments such as coughs or ulcer that could be associated with inflammation (Asl and Hosseinzadeh, 2008). There are also numerous scientific reports about the anti-inflammatory effects of licorice and compounds in different organs such as lung, skin, nervous system, liver, and cardiovascular system to name a few (Chu et al., 2013; Dohil, 2013 Kim et al., 2013b; Kang et al., 2015; Yu et al., 2015). The role of licorice in preventing breast cancer through modulating inflammatory pathways has also been studied. Comparative evaluation of three medicinally used licorice species showed that $G$. inflata followed by G. glabra and G. uralensis inhibited iNOS activity in macrophage cells. In this study, isoliquiritigenin and licochalcone A strongly blocked iNOS activity, suggesting the defining role of these two compounds in the anti-inflammatory effects of licorice species (Dunlap et al., 2015). Isoliquiritigenin was also shown to modulate COX-2 and iNOS and reduce PGE2 and nitric oxide levels and also exert antiproliferative chemopreventive effects in vitro and in vivo (Takahashi et al., 2004; Lau et al., 2010). Isoliquiritigenin has been shown to block colitisassociated tumorigenesis in animal models after 12 weeks of treatment through the suppression of PGE2 and interleukin-6 (IL-6) (Zhao et al., 2014). It was also reported that isoliquiritigenin exhibited growth inhibition and apoptosis in MCF-7 and MDA-MB-231 cells as well as in MDA-MB-231-inoculated nude mice through downregulating arachidonic acid metabolism enzymes such as COX-2 and deactivating phosphatidyl inositol 3 kinase/protein kinase B pathway (Li et al., 2013b). Additionally low concentrations of isoliquiritigenin might have breast cancer therapeutic benefits via inhibiting p38, phosphatidyl inositol 3 kinase/protein kinase $\mathrm{B}$, and NF- $\kappa \mathrm{B}$ pathways (Lorusso and Marech, 2013; Wang et al., 2013). Isoliquiritigenin inhibited phorbol ester-induced COX-2 expression in MCF-10A cells by modulating the ERK1/2 signaling pathway (Lau et al., 2010). Glabridin was reported to exert chemopreventive effects by attenuating angiogenesis via inhibiting NF-кB/IL-6/signal transducer and activator of transcription 3 signaling pathway in MDAMB-231 and Hs-578T cells (Mu et al., 2015).

4. Epigenetic Pathway. Licorice species and their related compounds have not been well studied for their potential to modulate epigenetic factors in breast cancer. It has been suggested that glabridin inhibits cancer stem cell-like properties of human breast cancer cells via modulation of micro RNA signaling (Jiang et al., 2016). In summary, licorice species have the potential to protect women from breast cancer; however, more rigorous studies with well-characterized/ standardized licorice extracts are needed before recommendations of long-term use for breast cancer chemoprevention.

\section{H. Silybum marianum (Milk Thistle)}

Milk thistle has a long tradition (2000 years) of use for liver and biliary disorders ["PDQ Milk Thistle" (http:// www.cancer.gov/about-cancer/treatment/cam/hp/milkthistle-pdq); Abenavoli et al., 2010; Vargas-Mendoza et al., 2014]. Currently, milk thistle and especially silymarin, a mixture of flavonolignans and precursor flavonoids contained in milk thistle seeds (Fig. 21), which are also used as galactagogues [Silybum marianum (Milk Thistle)], have been extensively studied as chemopreventive agents especially against hepatocarcinoma through antioxidant mechanisms (Abenavoli et al., 2010; Zapantis et al., 2012). Milk thistle could also be effective as a chemopreventive agent for breast cancer as outlined below (Agarwal et al., 2006; Malewicz et al., 2006; Cheung et al., 2010).

1. Hormonal Pathway. Weak estrogenic activities have been shown for silymarin in in vitro and in vivo studies [Silybum marianum (Milk Thistle)] (Seidlova-Wuttke 
et al., 2003; Pliskova et al., 2005; El-Shitany et al., 2010). The estrogenic activities of silymarin are at least in part mediated through the ER $\beta$-receptor; silibin B, taxifolin, and quercetin show some weak $\operatorname{ER} \beta$ estrogenic activity (Pliskova et al., 2005; El-Shitany et al., 2010; Powers and Setzer, 2015), although other studies reported no estrogenic activities for taxifolin and quercetin (Jefferson et al., 2002; Resende et al., 2013). The effect of dietary silymarin against mammary carcinogenesis was tested in a $N$-methyl- $N$-nitrosoureainduced Sprague-Dawley rat model. In contrast to the expected protective effect, the authors reported a modest increase in the number of $N$-methyl- $N$-nitrosoureainduced mammary tumors, suggesting a cautionary note for breast cancer prevention (Malewicz et al., 2006). Finally, silymarin has also been reported to inhibit aromatase activity with an $\mathrm{IC}_{50}$ of $6.7 \mu \mathrm{g} / \mathrm{ml}$ (Jeong et al., 1999); however, it is not known which constituents of silymarin are responsible for the aromatase inhibiting activity.

2. Chemical Pathway. Several studies report the antioxidant properties of silymarin that prevent toxininduced liver diseases and might also block estrogeninduced genotoxicity (Vargas-Mendoza et al., 2014). Strong inhibition of recombinant P450 1A1 has been shown for dehydrosilybin (Fig. 21) $\left(\mathrm{IC}_{50}=0.43 \mu \mathrm{M}\right)$, whereas silibinin only moderately inhibited the P450 $1 \mathrm{~A} 1$ activity $\left(\mathrm{IC}_{50}=23 \mu \mathrm{M}\right)$ (Dvorak et al., 2006). Silymarin also reduced benzo(a)pyrene (BaP)-induced hepatic $\mathrm{P} 4501 \mathrm{~A} / 1 \mathrm{~B}$ activity in an in vivo toxicity model (Kiruthiga et al., 2015). In addition, silymarin enhanced NQO1, sulfotransferase activities, and glutathione levels, leading to a reduction of BaP-induced toxicity in this animal model. There have been no reports on the effects of milk thistle or its bioactive compounds on estrogen metabolism.

3. Inflammatory Pathway. A number of studies support anti-inflammatory activity of silymarin and especially its major component, silibinin (Fig. 21) (Gazak et al., 2007; Deep and Agarwal, 2013). For example, silibinin treatment significantly reduced $\mathrm{NF}-\kappa \mathrm{B}$ activity, levels of IL- $1 \beta$, and TNF- $\alpha$ in inflammatory colitis models (Esmaily et al., 2011). Silymarin also suppressed NF- $\kappa$ B activation by a variety of inflammatory agents in cell culture (Manna et al., 1999; Polyak et al., 2010; Kim et al., 2013a). Mechanism of action studies showed inhibition of phosphorylation of $\mathrm{I} \kappa \mathrm{B} \alpha$ by silymarin, thus reducing NF- $\kappa \mathrm{B}$ activation and nuclear translocation (Fig. 36) (Kim et al., 2013a). Downregulation of TPA-induced COX-2 by silibinin (around $50 \mu \mathrm{M}$ ) was also observed in two breast cancer cell lines (Kim et al., 2009). These results demonstrate that silibinin has several anti-inflammatory properties in cells, including breast cell lines as well as in vivo; however, further studies are necessary to determine if the high concentrations used in these studies will translate into clinical activity.
4. Epigenetic Pathway. Silymarin has not been extensively studied for epigenetic effects and no studies in breast cancer models have been reported (Gerhauser, 2012 ; 2013). It has been reported that silibinin (80 or $160 \mathrm{mg} / \mathrm{kg} \mathrm{BW}$, gavage, for 5 weeks) reduced human hepatocellular xenograft growth, which was associated with increased histone acetylation of $\mathrm{H} 3$ and $\mathrm{H} 4$, indicating their potential role in hepatocellular carcinoma growth (Cui et al., 2009). In contrast, another study described silymarin (around $500 \mu \mathrm{M}$ ) as an activator of the deacetylase SIRT1 in human melanoma cells, (Li et al., 2007; Gerhauser, 2012). Because very high concentrations were used in these studies, their translation into clinical effects is questionable. In conclusion, several in vitro studies depict that silymarin and its constituents might influence breast cancer through the hormonal, chemical, inflammatory, and perhaps epigenetic pathways; however, it is not known yet whether these effects will translate into clinical effects. Also, more studies are needed to describe the exact bioactivities of individual silymarin compounds.

\section{Angelica sinensis (Dong Quai)}

The dried roots of Angelica sinensis (dong quai) have been used for centuries as women's tonic in traditional Chinese medicine, and the chemopreventive properties of this botanical are also well known (Wang et al., 2010). In Taiwan for example, 7 of the top 10 most frequently prescribed Chinese herbal products for breast cancer treatment contained dong quai (Lai et al., 2012). In traditional Chinese medicine, dong quai is usually used in combination with other herbs, and therefore many studies analyze the activity of these botanical combinations rather than dong quai and its bioactive compounds. Therefore, conclusive results about dong quai's bioactivity are limited (Jin et al., 2016). The chemopreventive properties of dong quai, which might lead to modulation of estrogen carcinogenesis, are described below.

1. Hormonal Pathway. As mentioned in section IV. A.3.a, whether dong quai has estrogenic activities is highly controversial (Liu et al., 2001; Lau et al., 2005; Circosta et al., 2006). Some studies describe proliferative activities of a dong quai extract in MCF-7 (ER $\alpha$ ) cells (Amato et al., 2002; Lau et al., 2005) and in BT20 (ER-negative cells) (Lau et al., 2005) and an increase in uterine weight in female Wistar rats (Circosta et al., 2006). Chang et al. (2006) demonstrated that one of dong quai's phytoconstituents, ferulic acid (100 nM) (Fig. 10), ER dependently stimulated MCF-7 breast cancer cell proliferation, likely through upregulation of ER $\alpha$-mRNA and HER2 oncogene expression. However, other investigations did not observe estrogenic activities or even described antiestrogenic effects (Zhang et al., 2005; Godecke et al., 2012). Therefore, the proliferative or estrogenic activities of dong quai might be dependent on the kind of extract, and further studies are necessary 
to delineate the estrogenic properties of dong quai and its phytochemicals. Aromatase modulating effects have not been reported for dong quai.

2. Chemical Pathway. Studies in breast cells are less known; however, phytochemicals of dong quai have been reported to modulate enzymes that are involved in estrogen metabolism. Z-ligustilide (5-50 $\mu \mathrm{M})$ (Fig. 10) has been demonstrated to significantly inhibit BaP-induced CYP1A1 upregulation in human keratinocytes $(\mathrm{Wu}$ et al., 2014). $Z$-ligustilide (50 $\mu \mathrm{M})$ did not activate or inhibit BaP-induced AhR translocation, but it induced Nrf2 translocation and transfection of si-Nrf2 abrogated the preventive effect of $Z$-ligustilide on $\mathrm{BaP}$ induced CYP1A1. Therefore, the authors concluded that $Z$-ligustilide reduced BaP-induced CYP1A1 upregulation likely through a Nrf2-mediated mechanism (Wu et al., 2014). $Z$-ligustilide (5-10 $\mu \mathrm{M})$ also induced Nrf2-dependent detoxification genes, such as hemeoxygenase and NQO1 in this cell line. Similarly, other studies demonstrated that lipophilic extracts of dong quai, such as the $\mathrm{CO}_{2}$ supercritical fluid-extract, which is rich in $Z$-ligustilide, induced NQO1 protein and activity, ARE-luciferase activity, and Nrf2 mRNA in hepatoma cells (Saw et al., 2013). Z-ligustilide was the main compound responsible for this activity and doubled the NQO1 activity at a concentration of $6.9 \pm 1.9 \mu \mathrm{M}$ and had a chemopreventive index of 10 in hepatoma cells (Dietz et al., 2008). Mechanism of action studies showed that $Z$-ligustilide alkylated cysteine residues in Keap1, thus enhancing Nrf2 translocation and AREluciferase activity (Fig. 35). This suggests that dong quai extracts that are rich in $Z$-ligustilide have the potential to induce detoxification enzymes and therefore might increase the detoxification of genotoxic estrogen metabolites (Fig. 34).

3. Inflammatory Pathway. Various in vitro and in vivo studies analyzed the inflammatory activity of dong quai and its phytochemicals. For example, the lipophilic supercritical $\mathrm{CO}_{2}$ extract of dong quai (1.1-17 $\mu \mathrm{g} / \mathrm{ml}$ ), which is rich in $Z$-ligustilide, and Z-ligustilide itself (6.3-50 $\mu \mathrm{M})$ suppressed LPS-induced NO production in macrophages (RAW 264.7 cells) (Saw et al., 2013). The extract $(17 \mu \mathrm{g} / \mathrm{ml})$ also significantly inhibited LPS-induced IL- $1 \beta$ in these macrophages. The volatile oil of dong quai $(0.176 \mathrm{ml} / \mathrm{kg} \mathrm{BW})$ inhibited LPS-induced acute inflammation in a rat model ( $\mathrm{Li}$ et al., 2016). Specifically, the oil decreased the levels of proinflammatory cytokines, such as TNF- $\alpha$ and IL- $1 \beta$; inflammatory mediators, such as PGE2 and NO; the inflammationrelated enzymes (iNOS and COX-2); and promoted the production of the anti-inflammatory cytokines IL-10. $Z$-ligustilide $(5-25 \mu \mathrm{M})$, one of the most abundant ingredients in the volatile oil, also significantly suppressed the production of NO, iNOS, PGE2, and TNF- $\alpha$ in LPS-induced inflammation in macrophages (RAW 264.7) (Su et al., 2011). Mechanism of action studies demonstrated that $Z$-ligustilide decreased the phosphorylation of IKK, thus reducing the cytosolic degradation of $\mathrm{I} \kappa \mathrm{B} \alpha$ and decreasing nuclear activation of NF- $\kappa \mathrm{B}$ (Fig. 36). In addition to its anti-inflammatory effects, $Z$-ligustilide also significantly reduced reactive oxygen species in this cell line. Not only $Z$-ligustilide $\left(\mathrm{IC}_{50}=25 \mu \mathrm{M}\right)$, but also another compound in dong quai, ferulic acid $\left(\mathrm{IC}_{50}=\right.$ $10 \mu \mathrm{M})$ (Fig. 10), inhibited NF- $\kappa \mathrm{B}$ luciferase activity in LPS and interferon- $\gamma$-stimulated murine macrophages (Chao et al., 2010) as well as decreased hydrogen peroxide-induced IL- $1 \beta$ and TNF- $\alpha$ mRNA in porcine chondrocytes (Chen et al., 2010a). An ethyl acetate extract of dong quai, which contains both compounds as major constituents, significantly reduced inflammatory markers, such as TNF- $\alpha$, in vitro (concentrations $\geq 5 \mu \mathrm{g} / \mathrm{ml}$ ) and in vivo $(1.56 \mathrm{mg} / \mathrm{kg}$ BW tube feeding for 1 week before LPS injection) inflammation models (Chao et al., 2010).

4. Epigenetic Pathway. There are very few studies on the influence of dong quai and its constituents on epigenetic pathways. Z-ligustilide $(50 \mu \mathrm{M})$ and the supercritical $\mathrm{CO}_{2}$ fluid extract of dong quai $(8.5 \mu \mathrm{g} / \mathrm{ml})$ significantly decreased the relative amount of methylated DNA in the Nrf2 gene promoter region, thus leading to enhanced Nrf2 mRNA and protein expression as well as to upregulation of its downstream target genes in TRAMP C1 prostate cancer cells ( $\mathrm{Su}$ et al., 2013; Guo et al., 2015b). In conclusion, several studies suggest that dong quai and its compounds, mainly $Z$-ligustilide and ferulic acid, might have the possibility to modulate estrogen carcinogenesis; however, conflicting data of dong quai on its effect of the hormonal pathway as well as limited data on its influence on estrogen metabolism and epigenetic pathways warrant further investigations before clinical studies can be performed.

\section{J. Others}

As mentioned in section IV.A.2.g, Epimedium species have a long history in treating sexual dysfunction and osteoporosis in traditional Chinese medicine and recent studies suggest chemopreventive properties (Ma et al., 2011). The aglycone icaritin and its metabolite desmethylicaritin (Fig. 30) demonstrated proliferative activities ( $1 \mathrm{nM}-10 \mu \mathrm{M})$ in breast cancer cells, MCF-7, potentially enhancing hormonal estrogen carcinogenesis (Wang and Lou, 2004). However, at higher concentrations $(>1 \mu \mathrm{M})$, icaritin inhibited estradiol induced MCF-7 cell proliferation (Tiong et al., 2012). Mechanism of action studies revealed that icaritin as an AhR agonist leads to destabilization of the $\mathrm{ER} \alpha$ protein and to subsequent reduction of estrogenic responses. This activity was confirmed in an in vivo model, in which icaritin treatment $(10 \mu \mathrm{mol} / \mathrm{kg}$ every 2 days for 9 weeks, i.p.) reduced ER $\alpha$ protein levels and estradiolstimulated breast cancer xenograft growth. Similarly, administration of the highest dose of an Epimedium extract $(5 \mathrm{~g} / \mathrm{kg})$ to ovariectomized athymic nude mice that 
were inoculated with MCF-7 breast cancer cells reduced ethinyl estradiol-induced xenograft growth and ER $\alpha$ protein content (Indran et al., 2014). None of the concentrations induced mammary tumors and only the lowest concentration of Epimedium extract (500 $\mathrm{mg} / \mathrm{kg} \mathrm{BW}$ ) induced uterine weight, suggesting that lower concentrations might be estrogenic and higher concentrations reduce estrogenic responses through destabilization of $\mathrm{ER} \alpha$. Icaritin might also have the potential to modulate estrogen metabolism, because icaritin has been shown to compete with $\mathrm{TCDD}$ at the $\mathrm{AhR}\left(\mathrm{IC}_{50} \approx 1 \mu \mathrm{M}\right)$ and to induce CYP1A1 (Tiong et al., 2012). Moreover, investigations with a water extract of herba Epimedii demonstrated antiinflammatory activities in macrophages as well as in an in vivo inflammation model (Oh et al., 2015). The extract inhibited NF- $\kappa \mathrm{B}$ activation and thus reduced iNOS and subsequent NO formation as well as reduced IL- 6 and IL-1 $\beta$. It also induced the detoxification enzyme heme oxygenase. Overall, these studies show that herba Epimedii might have the potential to modulate estrogen carcinogenesis; however, more studies describing details of the different extracts and influence on different estrogen metabolism enzymes are necessary to assess its overall effect on estrogen carcinogenesis. Chemopreventive properties have also been described for ginger and for flaxseed, which are likely due to antioxidant, anti-inflammatory, and induction of detoxification genes (Gan et al., 2013; Mason and Thompson, 2014). Clinical trials are needed to establish safety and efficacy (Baliga et al., 2011). In conclusion, many women's health botanicals have the potential to influence estrogen carcinogenesis through multiple pathways. However, more studies are needed to delineate the exact effects on different pathways and to standardize the extracts for further clinical studies.

\section{Botanicals/Phytochemicals with Potential Toxic Effects}

Most botanicals used for women's health have a long history of ethnobotanical use and are generally considered safe (van Breemen et al., 2008). However, it is important for women and their health practitioners to remember that most botanicals have not been rigorously tested for potential toxic effects, unlike FDA approved drugs. Some case studies of hepatotoxicity in particular have been reported for these botanicals (e.g., black cohosh), although these seem to be rare and probably the result of other health-related issues (Mahady et al., 2008; Teschke et al., 2013). Botanicaldrug interactions could also lead to toxicity through drug overdoses or decreased drug efficacy, and these effects are briefly reviewed below. More comprehensive reviews were recently published (van Breemen, 2015; Sprouse and van Breemen, 2016).
Because of the popularity of botanical dietary supplements (Lindstrom et al., 2014; Smith et al., 2015), it is likely that many consumers use herbal products together with other drugs. This is particularly noteworthy, because many users of botanical products do not mention the use of dietary supplements to their health care providers. Some prescription drugs, such as warfarin and digoxin, have a narrow therapeutic index and therefore botanical/drug interactions with these drugs could have detrimental consequences (de Lima Toccafondo Vieira and Huang, 2012). In fact, studies showed that around 20-25\% of all prescription drug users take herbal medicines concomitantly with conventional medications (Shord et al., 2009; de Lima Toccafondo Vieira and Huang, 2012). Because of the concurrent use of botanical products and prescription drugs, pharmacokinetic (de Lima Toccafondo Vieira and Huang, 2012; Gurley et al., 2012; Sprouse and van Breemen, 2016) and pharmacodynamic botanical-drug interactions (de Lima Toccafondo Vieira and Huang, 2012) are possible (Kennedy and Seely, 2010). The pharmacokinetic and pharmacodynamic botanical-drug interactions were reviewed recently in detail (Colalto, 2010; de Lima Toccafondo Vieira and Huang, 2012; Gurley et al., 2012; Sprouse and van Breemen, 2016). Therefore, mainly the major potential botanical-drug interactions based on pharmacological interferences of the aforementioned women's health botanicals/phytochemicals will be briefly reviewed.

Most of the reported drug-botanical interactions are due to interference with metabolism (pharmacokinetic effects). For example, cranberry juice was shown to inhibit the metabolism of the probe P450 3A4 substrate midazolam in healthy volunteers (Kim et al., 2011). Silybin was reported to inhibit P450 3A4 and 2E1 (Sprouse and van Breemen, 2016), although no effect of milk thistle extract on P450 3A4 activity was observed in a small clinical trial (Gurley et al., 2006). The same trial showed no effect of black cohosh on P450 3A4 activity. Similarly, there was no effect of valerian on marker substrates of $\mathrm{P} 450$ 3A4, 1A2, 2E1, and 2D6 (12 volunteers, $375 \mathrm{mg} /$ day, administered for 28 days) (Gurley et al., 2005; Colalto, 2010). Another clinical trial with 12 healthy volunteers showed induction of P450 3A4 after silymarin treatment (140 mg/day, 9 days) (Rajnarayana et al., 2004; Colalto, 2010). Mild pharmacokinetic interactions have also been described for licorice (Sprouse and van Breemen, 2016). The current information suggests that concomitant intake of botanicals used for women's health are not a major risk for drugs that are metabolized by P450s (Zadoyan and Fuhr, 2012); however, health practitioners should monitor potential drug/botanical interactions in their patients.

Some botanical drug interactions are due to pharmacological interactions. Chasteberry may interact with 
A<smiles>COc1cc(OC)c2ccc(=O)oc2c1C(=O)C=C(C)C</smiles>

B

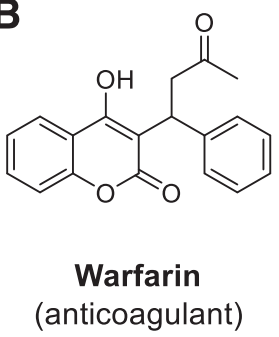

Fig. 38. (A) Coumarin derivative present in dong quai; (B) warfarin, anticoagulant drug.

drugs that antagonize the dopamine receptors and with oral contraceptive drugs (Daniele et al., 2005). Case reports describe that patients who took the anticoagulant warfarin (Fig. 38) together with Angelica sinensis (dong quai) extracts have an increased prothrombin time and showed widespread bruising (Fugh-Berman, 2000). Therefore, patients with clotting disorders or those that undergo anticoagulant therapy should be cautious with the concurrent use of dong quai products. The reason for the reported anticoagulant effect is likely that dong quai contains various coumarin derivatives (Fig. 38) that, like warfarin, inhibit the release of plasma clotting factor VII by vitamin $\mathrm{K}$ (Lowenthal and Birnbaum, 1969; Head, 2004). A caveat of these case reports is that the extract and specific phytochemicals are not well characterized (Fugh-Berman, 2000). Therefore, more research is warranted to delineate any potential interactive compounds as well as to use standardized extracts that give a consistent activity without showing drug interactions.

Licorice may lead to botanical-drug interactions and side effects after long-term use due to the sweet saponin compound, glycyrrhizin (or glycyrrhizinic acid), which is metabolized after oral administration to glycyrrhetinic acid (Fig. 27) (Fugh-Berman, 2000; Kao et al., 2014). Glycyrrhizin and especially glycyrrhetinic acid are

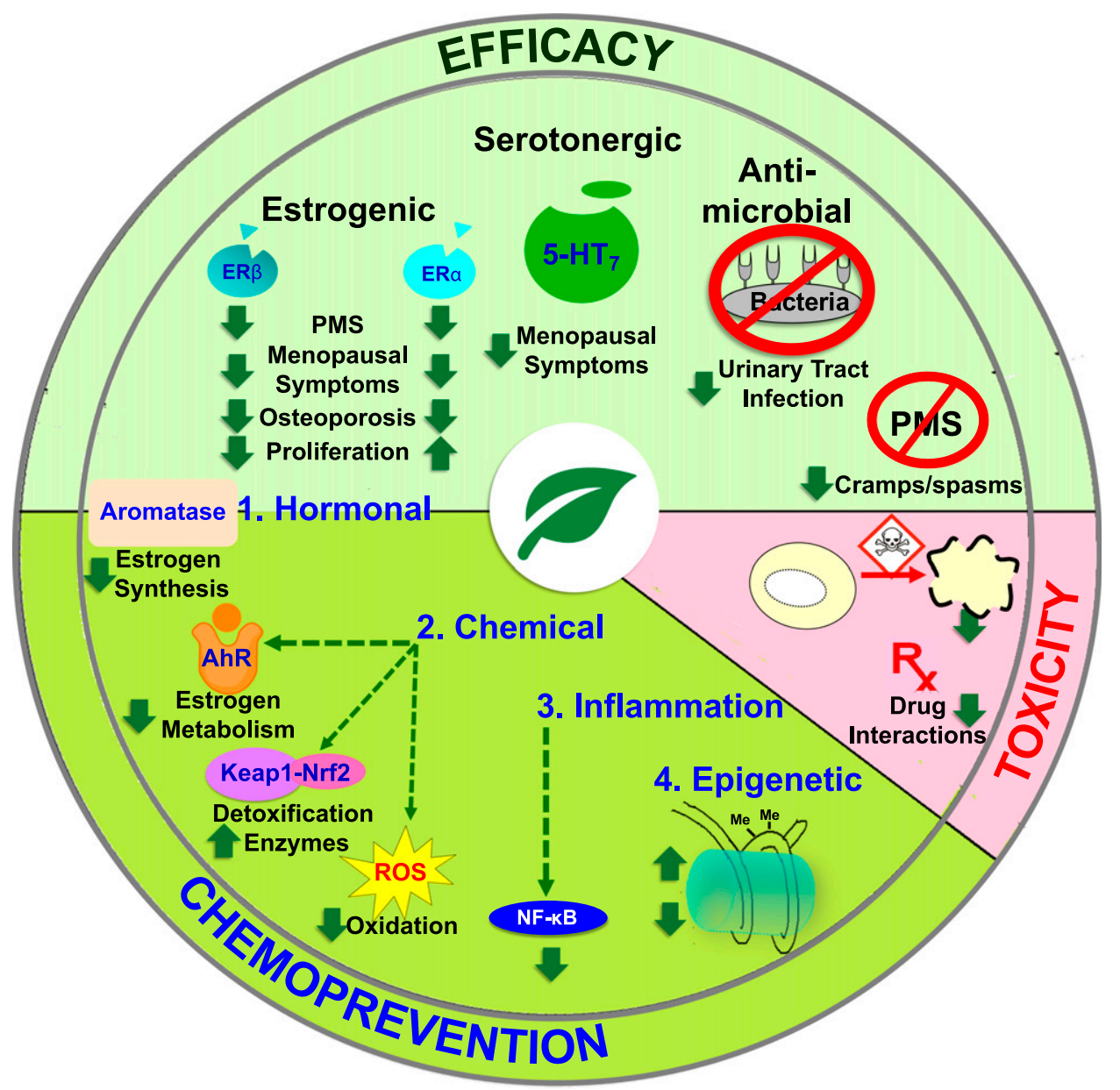

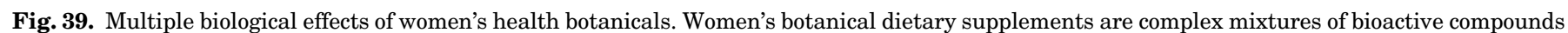

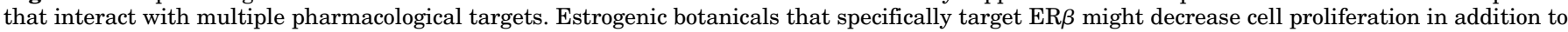

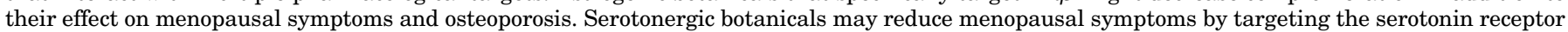

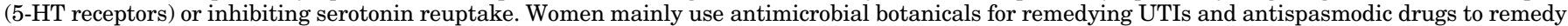

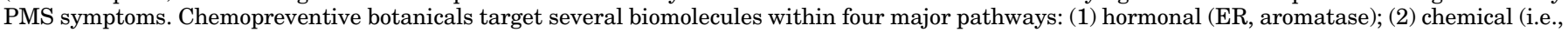

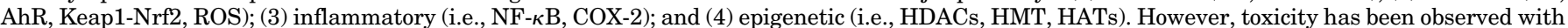

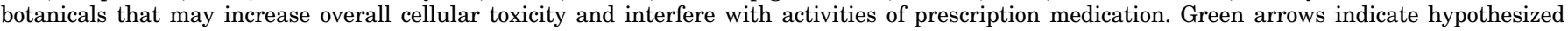
beneficial biological effects. 
potent inhibitors of the enzyme $11 \beta$-dehydrogenase, which is involved in the metabolism of cortisol (Kao et al., 2014). As a consequence, long-term usage of licorice products may lead to increased cortisol plasma levels, resulting in sodium retention, hypokalemia, edema that may eventually lead to hypertension and heart disease (Kao et al., 2014). When licorice is concurrently used with corticosteroid drugs, such as hydrocortisone or prednisolone, the corticosteroid treatment can be potentiated through licorice products (Teelucksingh et al., 1990; Fugh-Berman, 2000). A case report described profound hypokalemia after ingestion of small amounts of licorice contained in a laxative preparation taken together with an antihypertensive drug (not specified in the article) (Izzo et al., 2005). Because some antihypertensives and licorice may lead to an increase in potassium excretion, the combination might have led to an additive effect. Hypertension, edema, and hypokalemia were also reported after concurrent use of oral contraceptive and licorice products (Fugh-Berman, 2000). In addition to these adverse effects, glycyrrhizin and glycyrrhetinic acid have been reported to show many beneficial bioactivities that have been ascribed to licorice, such as anti-inflammatory, chemoprotective, antiulcerogenic, secretolytic, antiviral, and antimicrobial activities (Liu et al., 2013; Kao et al., 2014; Wang et al., 2015a).

\section{Conclusions and Future Directions}

Figure 39 summarizes the multiple effects of women's health botanicals described in this review. As women age, their botanical use changes from occasional use for UT infections, PMS, nausea associated with pregnancy, and lactation issues to more frequent use for menopausal symptoms, osteoporosis, and chemoprevention. Because botanicals are from natural sources they are perceived as harmless, although there are only a few studies on safety let alone efficacy. The increasing popularity of botanical supplements for women's health as well as the aging population requires rigorous clinical trials with standardized botanicals to determine efficacy. The effect of botanicals on hormone balance is likely dependent on age, and yet little information is available on efficacy in younger women versus older women. It is crucial that botanicals be fully standardized to botanical, phytochemical, and biological properties. In addition, the pharmacokinetic and distribution profile of the active constituents have to be determined to reach safe and efficacious concentrations in a clinical setting. Potential genetic polymorphisms in metabolism especially with the gut microbiota could have dramatic effects on the absorption and efficacy of botanicals. Drug-botanical interactions must be determined to avoid potentially toxic side effects or loss of activity of prescription drugs. Women must be encouraged to discuss their use of botanicals with their health care providers for assistance in managing all aspects of their health throughout their lives.

\section{Acknowledgments}

We very much appreciate the photographs of the plants in Fig. 1 provided by Dr. Doel Soejarto and Dan Lu, assistance with figures from Christina Sidorowych, and the helpful discussions with Dr. Richard van Breemen and Dr. Dejan Nikolic.

\section{Authorship Contributions}

Wrote or contributed to the writing of the manuscript: Dietz, Hajirahimkhan, Dunlap, and Bolton.

\section{References}

Abdel-Aziz H, Windeck T, Ploch M, and Verspohl EJ (2006) Mode of action of gingerols and shogaols on 5-HT3 receptors: binding studies, cation uptake by the receptor channel and contraction of isolated guinea-pig ileum. Eur $J$ Pharmacol 530:136-143.

Abenavoli L, Capasso R, Milic N, and Capasso F (2010) Milk thistle in liver diseases: past, present, future. Phytother Res 24:1423-1432.

AbouZahr C (2014) Progress and challenges in women's health: an analysis of levels and patterns of mortality and morbidity. Contraception 90(6, Suppl)S3-S13.

Adlercreutz H (2002) Phyto-oestrogens and cancer. Lancet Oncol 3:364-373.

Agarwal R, Agarwal C, Ichikawa H, Singh RP, and Aggarwal BB (2006) Anticancer potential of silymarin: from bench to bed side. Anticancer Res 26 (6B):4457-4498. Agarwal A, D'Souza P, Johnson TS, Dethe SM, and Chandrasekaran C (2014) Use of in vitro bioassays for assessing botanicals. Curr Opin Biotechnol 25:39-44.

Aghamiri V, Mirghafourvand M, Mohammad-Alizadeh-Charandabi S, and Nazemiyeh $\mathrm{H}$ (2016) The effect of Hop (Humulus lupulus L.) on early menopausal symptoms and hot flashes: A randomized placebo-controlled trial. Complement Ther Clin Pract 23: 130-135.

Ahmad B, Rehman MU, Amin I, Arif A, Rasool S, Bhat SA, Afzal I, Hussain I, Bilal S, and Mir $\mathrm{Mu}$ (2015) A review on pharmacological properties of zingerone (4-(4hydroxy-3-methoxyphenyl)-2-butanone). ScientificWorldJournal 2015:816364.

Alekel DL, Van Loan MD, Koehler KJ, Hanson LN, Stewart JW, Hanson KB, Kurzer MS, and Peterson CT (2010) The soy isoflavones for reducing bone loss (SIRBL) study: a 3-y randomized controlled trial in postmenopausal women. Am J Clin Nutr 91:218-230.

Ali BH, Blunden G, Tanira MO, and Nemmar A (2008) Some phytochemical, pharmacological and toxicological properties of ginger (Zingiber officinale Roscoe): a review of recent research. Food Chem Toxicol 46:409-420.

Allred CD, Allred KF, Ju YH, Clausen LM, Doerge DR, Schantz SL, Korol DL, Wallig MA, and Helferich WG (2004) Dietary genistein results in larger MNU-induced, estrogen-dependent mammary tumors following ovariectomy of Sprague-Dawley rats. Carcinogenesis 25:211-218.

Alzheimer's Association (2014) 2014 Alzheimer's disease facts and figures. Alzheimers Dement 10:e47-e92.

Amato P, Christophe S, and Mellon PL (2002) Estrogenic activity of herbs commonly used as remedies for menopausal symptoms. Menopause 9:145-150.

Amer MR, Cipriano GC, Venci JV, and Gandhi MA (2015) Safety of popular herbal supplements in lactating women. J Hum Lact 31:348-353.

Asl MN and Hosseinzadeh H (2008) Review of pharmacological effects of Glycyrrhiza sp. and its bioactive compounds. Phytother Res 22:709-724.

Ateba SB, Njamen D, Medjakovic S, Zehl M, Kaehlig H, Jungbauer A, and Krenn L (2014) Lupinalbin A as the most potent estrogen receptor $\alpha$ - and aryl hydrocarbon receptor agonist in Eriosema laurentii de Wild. (Leguminosae). BMC Complement Altern Med 14:294.

Atkinson C, Warren RM, Sala E, Dowsett M, Dunning AM, Healey CS, Runswick S Day NE, and Bingham SA (2004) Red-clover-derived isoflavones and mammographic breast density: a double-blind, randomized, placebo-controlled trial [ISRCTN42940165]. Breast Cancer Res 6:R170-R179.

Atteritano M, Pernice F, Mazzaferro S, Mantuano S, Frisina A, D'Anna R, Cannata ML, Bitto A, Squadrito F, Frisina N, et al. (2008) Effects of phytoestrogen genistein on cytogenetic biomarkers in postmenopausal women: 1 year randomized, placebocontrolled study. Eur J Pharmacol 589:22-26.

Baliga MS, Haniadka R, Pereira MM, D’Souza JJ, Pallaty PL, Bhat HP, and Popuri S (2011) Update on the chemopreventive effects of ginger and its phytochemicals. Crit Rev Food Sci Nutr 51:499-523.

Banerjee S, Li Y, Wang Z, and Sarkar FH (2008) Multi-targeted therapy of cancer by genistein. Cancer Lett 269:226-242.

Basse C and Arock M (2015) The increasing roles of epigenetics in breast cancer: Implications for pathogenicity, biomarkers, prevention and treatment. Int $J$ Cancer 137:2785-2794.

Bayles B and Usatine R (2009) Evening primrose oil. Am Fam Physician 80:1405-1408. Beavers KM, Serra MC, Beavers DP, Cooke MB, and Willoughby DS (2009) Soymilk supplementation does not alter plasma markers of inflammation and oxidative stress in postmenopausal women. Nutr Res 29:616-622.

Bent S, Padula A, Moore D, Patterson M, and Mehling W (2006) Valerian for sleep: a systematic review and meta-analysis. Am J Med 119:1005-1012.

Beral V; Million Women Study Collaborators (2003) Breast cancer and hormonereplacement therapy in the Million Women Study. Lancet 362:419-427.

Berger D, Schaffner W, Schrader E, Meier B, and Brattström A (2000) Efficacy of Vitex agnus castus L. extract Ze 440 in patients with pre-menstrual syndrome (PMS). Arch Gynecol Obstet 264:150-153.

Betz JM, Brown PN, and Roman MC (2011) Accuracy, precision, and reliability of chemical measurements in natural products research. Fitoterapia 82:44-52.

Bhavnani BR and Stanczyk FZ (2014) Pharmacology of conjugated equine estrogens: efficacy, safety and mechanism of action. J Steroid Biochem Mol Biol 142: $16-29$ 
Bialesova L, Novotna A, Macejova D, Brtko J, and Dvorak Z (2015) Agonistic effect of selected isoflavones on arylhydrocarbon receptor in a novel AZ-AhR transgenic gene reporter human cell line. Gen Physiol Biophys 34:331-334

Bianco NR, Chaplin LJ, and Montano MM (2005) Differential induction of quinone reductase by phytoestrogens and protection against oestrogen-induced DNA damage. Biochem $J$ 385:279-287.

Bisson J, McAlpine JB, Friesen JB, Chen SN, Graham J, and Pauli GF (2016) Can invalid bioactives undermine natural product-based drug discovery? $\mathrm{J}$ Med Chem 59:1671-1690.

Björnström L and Sjöberg M (2005) Mechanisms of estrogen receptor signaling: convergence of genomic and nongenomic actions on target genes. Mol Endocrinol 19:833-842.

Blei T, Soukup ST, Schmalbach K, Pudenz M, Möller FJ, Egert B, Wörtz N, Kurrat A, Müller D, Vollmer G, et al. (2015) Dose-dependent effects of isoflavone exposure during early lifetime on the rat mammary gland: Studies on estrogen sensitivity, isoflavone metabolism, and DNA methylation. Mol Nutr Food Res 59:270-283.

Blumenthal M; German Federal Institute for Drugs and Medical Devices Commission E (2000) Herbal Medicine: Expanded Commission E Monographs, Integrative Medicine Communications, Newton, MA.

Blumenthal M, Lindstom A, Ooyen C, and Lynch ME (2015) Herb supplement sales increase 6.8\% in 2014. HerbalGram 107:52-59.

Bolaños R, Del Castillo A, and Francia J (2010) Soy isoflavones versus placebo in the treatment of climacteric vasomotor symptoms: systematic review and meta-analysis. Menopause 17:660-666.

Bolton JL (2011) Mechanisms of estrogen carcinogenesis: modulation by botanical natural products, in Chemical Carcinogenesis (Penning TM ed) pp 75-93, Humana Press, New York.

Bonfield K, Amato E, Bankemper T, Agard H, Steller J, Keeler JM, Roy D, McCallum A, Paula S, and Ma L (2012) Development of a new class of aromatase inhibitors: design, synthesis and inhibitory activity of 3-phenylchroman-4-one (isoflavanone) derivatives. Bioorg Med Chem 20:2603-2613.

Boonmuen N, Gong P, Ali Z, Chittiboyina AG, Khan I, Doerge DR, Helferich WG, Carlson KE, Martin T, Piyachaturawat P, et al. (2016) Licorice root components in dietary supplements are selective estrogen receptor modulators with a spectrum of estrogenic and anti-estrogenic activities. Steroids 105:42-49.

Booth NL, Overk CR, Yao P, Burdette JE, Nikolic D, Chen SN, Bolton JL, van Breemen RB, Pauli GF, and Farnsworth NR (2006) The chemical and biologic profile of a red clover (Trifolium pratense L.) phase II clinical extract. J Altern Complement Med 12:133-139.

Borrelli F and Ernst E (2008) Black cohosh (Cimicifuga racemosa) for menopausal symptoms: a systematic review of its efficacy. Pharmacol Res 58:8-14.

Borrelli F and Ernst E (2010) Alternative and complementary therapies for the menopause. Maturitas 66:333-343.

Bosviel R, Dumollard E, Déchelotte P, Bignon YJ, and Bernard-Gallon D (2012) Can soy phytoestrogens decrease DNA methylation in BRCA1 and BRCA2 oncosuppressor genes in breast cancer? OMICS 16:235-244.

Brahmbhatt M, Gundala SR, Asif G, Shamsi SA, and Aneja R (2013) Ginger phytochemicals exhibit synergy to inhibit prostate cancer cell proliferation. Nutr Cancer 65:263-272.

Brass LM (2004) Hormone replacement therapy and stroke: clinical trials review. Stroke 35(11, Suppl 1):2644-2647.

Budeiri D, Li Wan Po A, and Dornan JC (1996) Is evening primrose oil of value in the treatment of premenstrual syndrome? Control Clin Trials 17:60-68.

Burdette CQ and Marcus RK (2013) Determination of isoflavone content in soy, red clover, and kudzu dietary supplement materials by liquid chromatography-particle beam/electron ionization mass spectrometry. J AOAC Int 96:925-932.

Byler S, Goldgar S, Heerboth S, Leary M, Housman G, Moulton K, and Sarkar S (2014) Genetic and epigenetic aspects of breast cancer progression and therapy. Anticancer Res 34:1071-1077.

Cao YF, He RR, Cao J, Chen JX, Huang T, and Liu Y (2012) Drug-Drug Interactions Potential of Icariin and Its Intestinal Metabolites via Inhibition of Intestinal UDP-Glucuronosyltransferases. Evid Based Complement Alternat Med 2012 . 395912.

Cavalieri E and Rogan E (2014) The molecular etiology and prevention of estrogeninitiated cancers: Ockham's Razor: Pluralitas non est ponenda sine necessitate. Plurality should not be posited without necessity. Mol Aspects Med 36:1-55.

Chadwick LR, Nikolic D, Burdette JE, Overk CR, Bolton JL, van Breemen RB, Fröhlich R, Fong HH, Farnsworth NR, and Pauli GF (2004) Estrogens and congeners from spent hops (Humulus lupulus). J Nat Prod 67:2024-2032.

Chadwick LR, Pauli GF, and Farnsworth NR (2006) The pharmacognosy of Humulus lupulus L. (hops) with an emphasis on estrogenic properties. Phytomedicine 13: $119-131$.

Chan HY and Leung LK (2003) A potential protective mechanism of soya isoflavones against 7,12-dimethylbenz[a]anthracene tumour initiation. Br J Nutr 90:457-465.

Chan HY, Wang H, and Leung LK (2003) The red clover (Trifolium pratense) isoflavone biochanin A modulates the biotransformation pathways of 7,12 dimethylbenz[a] anthracene. Br J Nutr 90:87-92.

Chandrasena RE, Edirisinghe PD, Bolton JL, and Thatcher GR (2008) Problematic detoxification of estrogen quinones by $\mathrm{NAD}(\mathrm{P}) \mathrm{H}$-dependent quinone oxidoreductase and glutathione-S-transferase. Chem Res Toxicol 21:1324-1329.

Chang CJ, Chiu JH, Tseng LM, Chang CH, Chien TM, Wu CW, and Lui WY (2006) Modulation of HER2 expression by ferulic acid on human breast cancer MCF7 cells. Eur J Clin Invest 36:588-596.

Chao WW, Hong YH, Chen ML, and Lin BF (2010) Inhibitory effects of Angelica sinensis ethyl acetate extract and major compounds on NF-kappaB transactivation activity and LPS-induced inflammation. $J$ Ethnopharmacol $\mathbf{1 2 9}$ 244-249.

Chen SN, Friesen JB, Webster D, Nikolic D, van Breemen RB, Wang ZJ, Fong HH, Farnsworth NR, and Pauli GF (2011) Phytoconstituents from Vitex agnus-castus fruits. Fitoterapia 82:528-533.
Chen SN, Li W, Fabricant DS, Santarsiero BD, Mesecar A, Fitzloff JF, Fong HH, and Farnsworth NR (2002) Isolation, structure elucidation, and absolute configuration of 26-deoxyactein from Cimicifuga racemosa and clarification of nomenclature associated with 27-deoxyactein. J Nat Prod 65:601-605.

Chen MP, Yang SH, Chou CH, Yang KC, Wu CC, Cheng YH, and Lin FH (2010a) The chondroprotective effects of ferulic acid on hydrogen peroxide-stimulated chondrocytes: inhibition of hydrogen peroxide-induced pro-inflammatory cytokines and metalloproteinase gene expression at the mRNA level. Inflamm Res 59:587-595.

Chen XP, Li W, Xiao XF, Zhang LL, and Liu CX (2013) Phytochemical and pharmacological studies on Radix Angelica sinensis. Chin J Nat Med 11:577-587.

Chen Y, Huang C, Zhou T, Zhang S, and Chen G (2010b) Biochanin A induction of sulfotransferases in rats. J Biochem Mol Toxicol 24:102-114.

Cheung CW, Gibbons N, Johnson DW, and Nicol DL (2010) Silibinin-a promising new treatment for cancer. Anticancer Agents Med Chem 10:186-195.

Chiang SS, Chang SP, and Pan TM (2011) Osteoprotective effect of Monascusfermented dioscorea in ovariectomized rat model of postmenopausal osteoporosis. J Agric Food Chem 59:9150-9157.

Chlebowski RT, Manson JE, Anderson GL, Cauley JA, Aragaki AK, Stefanick ML, Lane DS, Johnson KC, Wactawski-Wende J, Chen C, et al. (2013) Estrogen plus progestin and breast cancer incidence and mortality in the Women's Health Initiative Observational Study. J Natl Cancer Inst 105:526-535.

Cho YC, You SK, Kim HJ, Cho CW, Lee IS, and Kang BY (2010) Xanthohumol inhibits IL-12 production and reduces chronic allergic contact dermatitis. Int Immunopharmacol 10:556-561.

Choi SY, Ha TY, Ahn JY, Kim SR, Kang KS, Hwang IK, and Kim S (2008) Estrogenic activities of isoflavones and flavones and their structure-activity relationships. Planta Med 74:25-32.

Chou TC and Talalay P (1984) Quantitative analysis of dose-effect relationships: the combined effects of multiple drugs or enzyme inhibitors. Adv Enzyme Regul 22: $27-55$

Chu XT, de la Cruz J, Hwang SG, and Hong H (2014) Tumorigenic effects of endocrine-disrupting chemicals are alleviated by licorice (Glycyrrhiza glabra) root extract through suppression of AhR expression in mammalian cells. Asian Pac $J$ Cancer Prev 15:4809-4813.

Chu X, Jiang L, Wei M, Yang X, Guan M, Xie X, Wei J, Liu D, and Wang D (2013) Attenuation of allergic airway inflammation in a murine model of asthma by Licochalcone A. Immunopharmacol Immunotoxicol 35:653-661.

Chung MH, Kim DH, Na HK, Kim JH, Kim HN, Haegeman G, and Surh YJ (2014) Genistein inhibits phorbol ester-induced NF-kB transcriptional activity and COX-2 expression by blocking the phosphorylation of p65/RelA in human mammary epithelial cells. Mutat Res 768:74-83.

Circosta C, Pasquale RD, Palumbo DR, Samperi S, and Occhiuto F (2006) Estrogenic activity of standardized extract of Angelica sinensis. Phytother Res 20:665-669.

Clark IA and Vissel B (2013) Treatment implications of the altered cytokine-insulin axis in neurodegenerative disease. Biochem Pharmacol 86:862-871.

Cojocneanu Petric R, Braicu C, Raduly L, Zanoaga O, Dragos N, Monroig P, Dumitrascu D, and Berindan-Neagoe I (2015) Phytochemicals modulate carcinogenic signaling pathways in breast and hormone-related cancers. Onco Targets Ther 8:2053-2066.

Colalto C (2010) Herbal interactions on absorption of drugs: Mechanisms of action and clinical risk assessment. Pharmacol Res 62:207-227.

Colli MC, Bracht A, Soares AA, de Oliveira AL, Bôer CG, de Souza CG, and Peralta RM (2012) Evaluation of the efficacy of flaxseed meal and flaxseed extract in reducing menopausal symptoms. J Med Food 15:840-845.

Cometa MF, Parisi L, Palmery M, Meneguz A, and Tomassini L (2009) In vitro relaxant and spasmolytic effects of constituents from Viburnum prunifolium and HPLC quantification of the bioactive isolated iridoids. $J$ Ethnopharmacol 123: 201-207.

Constantinou AI, Lantvit D, Hawthorne M, Xu X, van Breemen RB, and Pezzuto JM (2001) Chemopreventive effects of soy protein and purified soy isoflavones on DMBAinduced mammary tumors in female Sprague-Dawley rats. Nutr Cancer 41:75-81.

Creatsas G, Deligeoroglou E, Zachari A, Loutradis D, Papadimitriou T, Miras K, and Aravantinos D (1990) Prostaglandins: PGF2 alpha, PGE2, 6-keto-PGF1 alpha and TXB2 serum levels in dysmenorrheic adolescents before, during and after treatment with oral contraceptives. Eur J Obstet Gynecol Reprod Biol 36 $292-298$

Cuendet M, Guo J, Luo Y, Chen S, Oteham CP, Moon RC, van Breemen RB, Marler LE, and Pezzuto JM (2010) Cancer chemopreventive activity and metabolism of isoliquiritigenin, a compound found in licorice. Cancer Prev Res (Phila) 3:221-232.

Cuendet M, Oteham CP, Moon RC, and Pezzuto JM (2006) Quinone reductase induction as a biomarker for cancer chemoprevention. J Nat Prod 69:460-463.

Cui W, Gu F, and Hu KQ (2009) Effects and mechanisms of silibinin on human hepatocellular carcinoma xenografts in nude mice. World $J$ Gastroenterol 15: $1943-1950$

Daniele C, Thompson Coon J, Pittler MH, and Ernst E (2005) Vitex agnus castus: a systematic review of adverse events. Drug Saf 28:319-332.

Dante G, Bellei G, Neri I, and Facchinetti F (2014) Herbal therapies in pregnancy: what works? Curr Opin Obstet Gynecol 26:83-91.

Davidson E, Zimmermann BF, Jungfer E, and Chrubasik-Hausmann S (2014) Prevention of urinary tract infections with vaccinium products. Phytother Res 28: $465-470$

Dawood MY (2006) Primary dysmenorrhea: advances in pathogenesis and management. Obstet Gynecol 108:428-441.

de Arriba SG, Naser B, and Nolte KU (2013) Risk assessment of free hydroquinone derived from Arctostaphylos Uva-ursi folium herbal preparations. Int J Toxicol 32: $442-453$

de la Cruz MS, Sarfaty M, and Wender RC (2014) An update on breast cancer screening and prevention. Prim Care 41:283-306.

de Lima Toccafondo Vieira M and Huang SM (2012) Botanical-drug interactions: a scientific perspective. Planta Med 78:1400-1415. 
Deep G and Agarwal R (2013) Targeting tumor microenvironment with silibinin: promise and potential for a translational cancer chemopreventive strategy. Curr Cancer Drug Targets 13:486-499.

Delmanto A, Nahas-Neto J, Traiman P, Uemura G, Pessoa EC, and Nahas EA (2013) Effects of soy isoflavones on mammographic density and breast parenchyma in postmenopausal women: a randomized, double-blind, placebo-controlled clinical trial. Menopause 20:1049-1054

Delmonte P, Perry J, and Rader JI (2006) Determination of isoflavones in dietary supplements containing soy, Red Clover and kudzu: extraction followed by basic or acid hydrolysis. $J$ Chromatogr A 1107:59-69.

Depypere HT and Comhaire FH (2014) Herbal preparations for the menopause: beyond isoflavones and black cohosh. Maturitas 77:191-194.

Deroo BJ and Buensuceso AV (2010) Minireview: Estrogen receptor-beta: mechanistic insights from recent studies. Mol Endocrinol 24:1703-1714.

Dew TP and Williamson G (2013) Controlled flax interventions for the improvement of menopausal symptoms and postmenopausal bone health: a systematic review. Menopause 20:1207-1215.

Dewanjee S, Gangopadhyay M, Bhattacharya N, Khanra R, and Dua TK (2015) Bioautography and its scope in the field of natural product chemistry. $J$ Pharm Anal 5:75-84.

Diel P, Geis RB, Caldarelli A, Schmidt S, Leschowsky UL, Voss A, and Vollmer G (2004a) The differential ability of the phytoestrogen genistein and of estradiol to induce uterine weight and proliferation in the rat is associated with a substance specific modulation of uterine gene expression. Mol Cell Endocrinol 221: 21-32.

Diel P, Thomae RB, Caldarelli A, Zierau O, Kolba S, Schmidt S, Schwab P, Metz P, and Vollmer G (2004b) Regulation of gene expression by 8-prenylnaringenin in uterus and liver of Wistar rats. Planta Med 70:39-44.

Dietz B and Bolton JL (2007) Botanical dietary supplements gone bad. Chem Res Toxicol 20:586-590

Dietz BM and Bolton JL (2013) Anti-inflammatory botanical dietary supplements for women's health: role in breast cancer prevention? in Inflammation, Oxidative Stress, and Cancer: Dietary Approaches for Cancer Chemprevention (Kong ANT ed) pp 529-548, CRC Press, Boca Raton, FL.

Dietz BM, Hagos GK, Eskra JN, Wijewickrama GT, Anderson JR, Nikolic D, Guo J, Wright B, Chen SN, Pauli GF, et al. (2013) Differential regulation of detoxification enzymes in hepatic and mammary tissue by hops (Humulus lupulus) in vitro and in vivo. Mol Nutr Food Res 57:1055-1066.

Dietz BM, Kang YH, Liu G, Eggler AL, Yao P, Chadwick LR, Pauli GF, Farnsworth NR, Mesecar AD, van Breemen RB, et al. (2005a) Xanthohumol isolated from Humulus lupulus Inhibits menadione-induced DNA damage through induction of quinone reductase. Chem Res Toxicol 18:1296-1305.

Dietz BM, Liu D, Hagos GK, Yao P, Schinkovitz A, Pro SM, Deng S, Farnsworth NR Pauli GF, van Breemen RB, et al. (2008) Angelica sinensis and its alkylphthalides induce the detoxification enzyme NAD(P)H: quinone oxidoreductase 1 by alkylating Keap1. Chem Res Toxicol 21:1939-1948.

Dietz BM, Mahady GB, Pauli GF, and Farnsworth NR (2005b) Valerian extract and valerenic acid are partial agonists of the 5-HT5a receptor in vitro. Brain Res Mol Brain Res 138:191-197.

Dijsselbloem N, Vanden Berghe W, De Naeyer A, and Haegeman G (2004) Soy isoflavone phyto-pharmaceuticals in interleukin-6 affections. Multi-purpose nutraceuticals at the crossroad of hormone replacement, anti-cancer and antiinflammatory therapy. Biochem Pharmacol 68:1171-1185.

Ding M, Leach M, and Bradley H (2013) The effectiveness and safety of ginger for pregnancy-induced nausea and vomiting: a systematic review. Women Birth 26 e26-e30.

Dini A, Migliuolo G, Rastrelli L, Saturnino P, and Schettino O (1994) ChemicalComposition of Lepidium-Meyenii. Food Chem 49:347-349.

Djaldetti $\mathrm{M}$ and Bessler $\mathrm{H}$ (2014) Modulators affecting the immune dialogue between human immune and colon cancer cells. World J Gastrointest Oncol 6:129-138.

Dohil MA (2013) Natural ingredients in atopic dermatitis and other inflammatory skin disease. J Drugs Dermatol 12(9, Suppl)s128-s132.

Dong S, Inoue A, Zhu Y, Tanji M, and Kiyama R (2007) Activation of rapid signaling pathways and the subsequent transcriptional regulation for the proliferation of breast cancer MCF-7 cells by the treatment with an extract of Glycyrrhiza glabra root. Food Chem Toxicol 45:2470-2478.

Dong JY and Qin LQ (2011) Soy isoflavones consumption and risk of breast cancer incidence or recurrence: a meta-analysis of prospective studies. Breast Cancer Res Treat 125:315-323.

Donovan TJ and Buchanan K (2012) Medications for increasing milk supply in mothers expressing breastmilk for their preterm hospitalised infants. Cochrane Database Syst Rev 3:CD005544.

Dorn C, Heilmann J, and Hellerbrand C (2012) Protective effect of xanthohumol on toxin-induced liver inflammation and fibrosis. Int J Clin Exp Pathol 5:29-36.

Dorn C, Massinger S, Wuzik A, Heilmann J, and Hellerbrand C (2013) Xanthohumol suppresses inflammatory response to warm ischemia-reperfusion induced liver injury. Exp Mol Pathol 94:10-16.

Du J, Bai B, Kuang X, Yu Y, Wang C, Ke Y, Xu Y, Tzang AH, and Qian ZM (2006) Ligustilide inhibits spontaneous and agonists- or $\mathrm{K}+$ depolarization-induced contraction of rat uterus. J Ethnopharmacol 108:54-58.

Dunlap TL, Wang S, Simmler C, Chen SN, Pauli GF, Dietz BM, and Bolton JL (2015) Differential effects of glycyrrhiza species on genotoxic estrogen metabolism: licochalcone A downregulates P450 1B1, whereas isoliquiritigenin stimulates it. Chem Res Toxicol 28:1584-1594.

Dvorák Z, Vrzal R, and Ulrichová J (2006) Silybin and dehydrosilybin inhibit cytochrome P450 1A1 catalytic activity: a study in human keratinocytes and human hepatoma cells. Cell Biol Toxicol 22:81-90.

Edmunds KM, Holloway AC, Crankshaw DJ, Agarwal SK, and Foster WG (2005) The effects of dietary phytoestrogens on aromatase activity in human endometrial stromal cells. Reprod Nutr Dev 45:709-720.
Einbond LS, Shimizu M, Nuntanakorn P, Seter C, Cheng R, Jiang B, Kronenberg F, Kennelly EJ, and Weinstein IB (2006) Actein and a fraction of black cohosh potentiate antiproliferative effects of chemotherapy agents on human breast cancer cells. Planta Med 72:1200-1206.

El-Shitany NA, Hegazy S, and El-Desoky K (2010) Evidences for antiosteoporotic and selective estrogen receptor modulator activity of silymarin compared with ethinylestradiol in ovariectomized rats. Phytomedicine 17:116-125.

Ellmann S, Sticht H, Thiel F, Beckmann MW, Strick R, and Strissel PL (2009) Estrogen and progesterone receptors: from molecular structures to clinical targets. Cell Mol Life Sci 66:2405-2426.

Erkkola R, Vervarcke S, Vansteelandt S, Rompotti P, De Keukeleire D, and Heyerick A (2010) A randomized, double-blind, placebo-controlled, cross-over pilot study on the use of a standardized hop extract to alleviate menopausal discomforts. Phy tomedicine 17:389-396.

Esmaily H, Vaziri-Bami A, Miroliaee AE, Baeeri M, and Abdollahi M (2011) The correlation between $\mathrm{NF}-\mathrm{\kappa B}$ inhibition and disease activity by coadministration of silibinin and ursodeoxycholic acid in experimental colitis. Fundam Clin Pharmacol 25:723-733.

Farmer C, Lapointe J, and Palin MF (2014) Effects of the plant extract silymarin on prolactin concentrations, mammary gland development, and oxidative stress in gestating gilts. J Anim Sci 92:2922-2930.

Farnsworth NR, Krause EC, Bolton JL, Pauli GF, van Breemen RB, and Graham JG (2008) The University of Illinois at Chicago/National Institutes of Health Center for Botanical Dietary Supplements Research for Women's Health: from plant to clinical use. Am J Clin Nutr 87:504S-508S.

Farzaneh F, Fatehi S, Sohrabi MR, and Alizadeh K (2013) The effect of oral evening primrose oil on menopausal hot flashes: a randomized clinical trial. Arch Gynecol Obstet 288:1075-1079.

Fiesel A, Gessner DK, Most E, and Eder K (2014) Effects of dietary polyphenol-rich plant products from grape or hop on pro-inflammatory gene expression in the intestine, nutrient digestibility and faecal microbiota of weaned pigs. BMC Vet Res 10:196.

Fillet M and Frédérich M (2015) The emergence of metabolomics as a key discipline in the drug discovery process. Drug Discov Today Technol 13:19-24.

Folwarczna J, Zych M, Nowińska B, and Pytlik M (2014) Effects of fenugreek (Trigonella foenum-graecum L.) seed on bone mechanical properties in rats. Eur Rev Med Pharmacol Sci 18:1937-1947.

Foo LY, Lu Y, Howell AB, and Vorsa N (2000) The structure of cranberry proanthocyanidins which inhibit adherence of uropathogenic P-fimbriated Escherichia coli in vitro. Phytochemistry 54:173-181.

Forinash AB, Yancey AM, Barnes KN, and Myles TD (2012) The use of galactogogues in the breastfeeding mother. Ann Pharmacother 46:1392-1404.

Freeman EW (2010) Therapeutic management of premenstrual syndrome. Expert Opin Pharmacother 11:2879-2889.

Froyen EB, Reeves JL, Mitchell AE, and Steinberg FM (2009) Regulation of phase II enzymes by genistein and daidzein in male and female Swiss Webster mice. J Med Food 12:1227-1237.

Froyen EB and Steinberg FM (2011) Soy isoflavones increase quinone reductase in hepa-1c1c7 cells via estrogen receptor beta and nuclear factor erythroid 2-related factor 2 binding to the antioxidant response element. $J$ Nutr Biochem 22:843-848 Fu SW, Zeng GF, Zong SH, Zhang ZY, Zou B, Fang Y, Lu L, and Xiao DQ (2014) Systematic review and meta-analysis of the bone protective effect of phytoestrogens on osteoporosis in ovariectomized rats. Nutr Res 34:467-477.

Fugh-Berman A (2000) Herb-drug interactions. Lancet 355:134-138.

Gabay MP (2002) Galactogogues: medications that induce lactation. J Hum Lact 18 $274-279$.

Gan FF, Ling H, Ang X, Reddy SA, Lee SS, Yang H, Tan SH, Hayes JD, Chui WK and Chew EH (2013) A novel shogaol analog suppresses cancer cell invasion and inflammation, and displays cytoprotective effects through modulation of NF-kB and Nrf2-Keap1 signaling pathways. Toxicol Appl Pharmacol 272:852-862.

Ganai AA, Khan AA, Malik ZA, and Farooqi H (2015) Genistein modulates the expression of NF-кB and MAPK (p-38 and ERK1/2), thereby attenuating d-Galactosamine induced fulminant hepatic failure in Wistar rats. Toxicol Appl Pharmacol 283: 139-146.

Ganesan P, Ko HM, Kim IS, and Choi DK (2015) Recent trends in the development of nanophytobioactive compounds and delivery systems for their possible role in reducing oxidative stress in Parkinson's disease models. Int J Nanomedicine 10: $6757-6772$

Gao F, Wei D, Bian T, Xie P, Zou J, Mu H, Zhang B, and Zhou X (2012) Genistein attenuated allergic airway inflammation by modulating the transcription factors T-bet, GATA-3 and STAT-6 in a murine model of asthma. Pharmacology 89:229-236. Garcia-Cazarin ML, Wambogo EA, Regan KS, and Davis CD (2014) Dietary supplement research portfolio at the NIH, 2009-2011. J Nutr 144:414-418.

Gazák R, Walterová D, and Kren V (2007) Silybin and silymarin-new and emerging applications in medicine. Curr Med Chem 14:315-338.

Geller SE, Shulman LP, van Breemen RB, Banuvar S, Zhou Y, Epstein G, Hedayat S, Nikolic D, Krause EC, Piersen CE, et al. (2009) Safety and efficacy of black cohosh and red clover for the management of vasomotor symptoms: a randomized controlled trial. Menopause 16:1156-1166.

Gerhauser C (2012) Cancer cell metabolism, epigenetics and the potential influence of dietary components - A perspective. Biomed Res-India 23:69-89.

Gerhauser C (2013) Cancer chemoprevention and nutriepigenetics: state of the art and future challenges. Top Curr Chem 329:73-132.

Gerhäuser C, Alt A, Heiss E, Gamal-Eldeen A, Klimo K, Knauft J, Neumann I, Scherf HR, Frank N, Bartsch H, et al. (2002) Cancer chemopreventive activity of Xanthohumol, a natural product derived from hop. Mol Cancer Ther 1:959-969.

Gertsch J (2011) Botanical drugs, synergy, and network pharmacology: forth and back to intelligent mixtures. Planta Med 77:1086-1098.

Giacinti L, Claudio PP, Lopez M, and Giordano A (2006) Epigenetic information and estrogen receptor alpha expression in breast cancer. Oncologist 11:1-8. 
Giacosa A, Morazzoni P, Bombardelli E, Riva A, Bianchi Porro G, and Rondanelli M (2015) Can nausea and vomiting be treated with ginger extract? Eur Rev Med Pharmacol Sci 19:1291-1296.

Girman A, Lee R, and Kligler B (2003) An integrative medicine approach to premenstrual syndrome. Am J Obstet Gynecol 188(5, Suppl):S56-S65.

Gödecke T, Yao P, Napolitano JG, Nikolić D, Dietz BM, Bolton JL, van Breemen RB, Farnsworth NR, Chen SN, Lankin DC, et al. (2012) Integrated standardization concept for Angelica botanicals using quantitative NMR. Fitoterapia 83:18-32.

Gong L, Li Y, Nedeljkovic-Kurepa A, and Sarkar FH (2003) Inactivation of NF-kappaB by genistein is mediated via Akt signaling pathway in breast cance cells. Oncogene 22:4702-4709.

Gonzales GF (2012) Ethnobiology and ethnopharmacology of Lepidium meyenii (Maca), a plant from the Peruvian Highlands. Evid Based Complement Alternat Med 2012:193496.

Greenlee H, Abascal K, Yarnell E, and Ladas E (2007) Clinical applications of Silybum marianum in oncology. Integr Cancer Ther 6:158-165.

Gross-Steinmeyer K, Stapleton PL, Tracy JH, Bammler TK, Strom SC, Buhler DR, and Eaton DL (2009) Modulation of aflatoxin B1-mediated genotoxicity in primary cultures of human hepatocytes by diindolylmethane, curcumin, and xanthohumols. Toxicol Sci 112:303-310.

Guay DR (2009) Cranberry and urinary tract infections. Drugs 69:775-807.

Guerra MC, Speroni E, Broccoli M, Cangini M, Pasini P, Minghett A, Crespi-Perellino N, Mirasoli M, Cantelli-Forti G, and Paolini M (2000) Comparison between chinese medical herb Pueraria lobata crude extract and its main isoflavone puerarin antioxidant properties and effects on rat liver CYP-catalysed drug metabolism. Life Sci 67:2997-3006.

Guo Y, Su ZY, and Kong AT (2015a) Current perspectives on epigenetic modification by dietary chemopreventive and herbal phytochemicals. Curr Pharmacol Rep 1:245-257.

Guo Y, Yu S, Zhang C, and Kong AN (2015b) Epigenetic regulation of Keap1-Nrf2 signaling. Free Radic Biol Med 88 (Pt B):337-349.

Gupta SC, Tyagi AK, Deshmukh-Taskar P, Hinojosa M, Prasad S, and Aggarwal BB (2014) Downregulation of tumor necrosis factor and other proinflammatory biomarkers by polyphenols. Arch Biochem Biophys 559:91-99.

Gurley BJ, Fifer EK, and Gardner Z (2012) Pharmacokinetic herb-drug interactions (part 2): drug interactions involving popular botanical dietary supplements and their clinical relevance. Planta Med 78:1490-1514.

Gurley BJ, Gardner SF, Hubbard MA, Williams DK, Gentry WB, Khan IA, and Shah A (2005) In vivo effects of goldenseal, kava kava, black cohosh, and valerian on human cytochrome P450 1A2, 2D6, 2E1, and 3A4/5 phenotypes. Clin Pharmacol Ther 77:415-426.

Gurley B, Hubbard MA, Williams DK, Thaden J, Tong Y, Gentry WB, Breen P, Carrier DJ, and Cheboyina S (2006) Assessing the clinical significance of botanical supplementation on human cytochrome P450 3A activity: comparison of a milk thistle and black cohosh product to rifampin and clarithromycin. J Clin Pharmacol 46:201-213.

Hajirahimkhan A, Dietz BM, and Bolton JL (2013a) Botanical modulation of menopausal symptoms: mechanisms of action? Planta Med 79:538-553.

Hajirahimkhan A, Simmler C, Dong H, Lantvit DD, Li G, Chen SN, Nikolić D, Pauli GF, van Breemen RB, Dietz BM, et al. (2015) Induction of NAD(P)H:quinone oxidoreductase 1 (NQO1) by Glycyrrhiza species used for women's health: differential effects of the Michael acceptors isoliquiritigenin and licochalcone A. Chem Res Toxicol 28:2130-2141.

Hajirahimkhan A, Simmler C, Yuan Y, Anderson JR, Chen SN, Nikolić D, Dietz BM, Pauli GF, van Breemen RB, and Bolton JL (2013b) Evaluation of estrogenic activity of licorice species in comparison with hops used in botanicals for menopausal symptoms. PLoS One 8:e67947.

Hall E, Frey BN, and Soares CN (2011) Non-hormonal treatment strategies for vasomotor symptoms: a critical review. Drugs 71:287-304.

Han EH, Kim JY, and Jeong HG (2006) Effect of biochanin A on the aryl hydrocarbon receptor and cytochrome P450 1A1 in MCF-7 human breast carcinoma cells. Arch Pharm Res 29:570-576.

Harikumar KB, Kunnumakkara AB, Ahn KS, Anand P, Krishnan S, Guha S, and Aggarwal BB (2009) Modification of the cysteine residues in IkappaBalpha kinase and NF-kappaB (p65) by xanthohumol leads to suppression of NF-kappaBregulated gene products and potentiation of apoptosis in leukemia cells. Blood 113 2003-2013.

Harris RE, Casto BC, and Harris ZM (2014) Cyclooxygenase-2 and the inflammogenesis of breast cancer. World J Clin Oncol 5:677-692.

Hasegawa E, Nakagawa S, Sato M, Tachikawa E, and Yamato S (2013) Effect of polyphenols on production of steroid hormones from human adrenocortical NCI H295R cells. Biol Pharm Bull 36:228-237.

Hasper I, Ventskovskiy BM, Rettenberger R, Heger PW, Riley DS, and KaszkinBettag M (2009) Long-term efficacy and safety of the special extract ERr 731 of Rheum rhaponticum in perimenopausal women with menopausal symptoms. Menopause 16:117-131.

He Z, Chen R, Zhou Y, Geng L, Zhang Z, Chen S, Yao Y, Lu J, and Lin S (2009) Treatment for premenstrual syndrome with Vitex agnus castus: A prospective, randomized, multi-center placebo controlled study in China. Maturitas 63:99-103.

Head K (2004) Monograph. Angelica sinensis. Altern Med Rev 9:429-433.

Heger M, Ventskovskiy BM, Borzenko I, Kneis KC, Rettenberger R, Kaszkin-Bettag M, and Heger PW (2006) Efficacy and safety of a special extract of Rheum rhaponticum (ERr 731) in perimenopausal women with climacteric complaints: a 12-week randomized, double-blind, placebo-controlled trial. Menopause 13:744-759.

Helle J, Kräker K, Bader MI, Keiler AM, Zierau O, Vollmer G, Welsh J, and Kretzschmar G (2014) Assessment of the proliferative capacity of the flavanones 8-prenylnaringenin, 6-(1.1-dimethylallyl)naringenin and naringenin in MCF-7 cells and the rat mammary gland. Mol Cell Endocrinol 392:125-135.

Hemachandra LP, Madhubhani P, Chandrasena R, Esala P, Chen SN, Main M, Lankin DC, Scism RA, Dietz BM, Pauli GF, et al. (2012) Hops (Humulus lupulus) inhibits oxidative estrogen metabolism and estrogen-induced malignant transformation in human mammary epithelial cells (MCF-10A). Cancer Prev Res (Phila) 5:73-81.

Henderson MC, Miranda CL, Stevens JF, Deinzer ML, and Buhler DR (2000) In vitro inhibition of human P450 enzymes by prenylated flavonoids from hops, Humulus lupulus. Xenobiotica 30:235-251.

Hervouet E, Cartron PF, Jouvenot M, and Delage-Mourroux R (2013) Epigenetic regulation of estrogen signaling in breast cancer. Epigenetics 8:237-245.

Heyerick A, Vervarcke S, Depypere H, Bracke M, and De Keukeleire D (2006) A first prospective, randomized, double-blind, placebo-controlled study on the use of a standardized hop extract to alleviate menopausal discomforts. Maturitas 54: 164-175.

Hirata JD, Swiersz LM, Zell B, Small R, and Ettinger B (1997) Does dong quai have estrogenic effects in postmenopausal women? A double-blind, placebo-controlled trial. Fertil Steril 68:981-986.

Hong KW, Ko KP, Ahn Y, Kim CS, Park SJ, Park JK, Kim SS, and Kim Y (2012) Epidemiological profiles between equol producers and nonproducers: a genomewide association study of the equol-producing phenotype. Genes Nutr 7 567-574.

Hong YH, Wang SC, Hsu C, Lin BF, Kuo YH, and Huang CJ (2011) Phytoestrogenic compounds in alfalfa sprout (Medicago sativa) beyond coumestrol. J Agric Food Chem 59:131-137.

Hopkins AL (2008) Network pharmacology: the next paradigm in drug discovery. Nat Chem Biol 4:682-690.

Hook IL (2014) Danggui to Angelica sinensis root: are potential benefits to European women lost in translation? A review. J Ethnopharmacol 152:1-13.

Horia E and Watkins BA (2007) Complementary actions of docosahexaenoic acid and genistein on COX-2, PGE2 and invasiveness in MDA-MB-231 breast cancer cells. Carcinogenesis 28:809-815.

Hougee S, Faber J, Sanders A, Berg WB, Garssen J, Smit HF, and Hoijer MA (2006) Selective inhibition of COX-2 by a standardized $\mathrm{CO} 2$ extract of Humulus lupulus in vitro and its activity in a mouse model of zymosan-induced arthritis. Planta Med 72:228-233.

Howe LR (2007) Inflammation and breast cancer. Cyclooxygenase/prostaglandin signaling and breast cancer. Breast Cancer Res 9:210.

Hsieh CY, Santell RC, Haslam SZ, and Helferich WG (1998) Estrogenic effects of genistein on the growth of estrogen receptor-positive human breast cancer (MCF-7) cells in vitro and in vivo. Cancer Res 58:3833-3838.

Hu Y, Hou TT, Xin HL, Zhang QY, Zheng HC, Rahman K, and Qin LP (2007a) Estrogen-like activity of volatile components from Vitex rotundifolia L. Indian $J$ Med Res 126:68-72.

Hu C, Liu H, Du J, Mo B, Qi H, Wang X, Ye S, and Li Z (2009) Estrogenic activities of extracts of Chinese licorice (Glycyrrhiza uralensis) root in MCF-7 breast cancer cells. J Steroid Biochem Mol Biol 113:209-216.

Hu Y, Zhang QY, Hou TT, Xin HL, Zheng HC, Rahman K, and Qin LP (2007b) Estrogen-like activities in Vitex species from China determined by a cell based proliferation assay. Pharmazie 62:872-875.

Huang B, Warner M, and Gustafsson JA (2015) Estrogen receptors in breast carcinogenesis and endocrine therapy. Mol Cell Endocrinol 418:240-244.

Hwang KA and Choi KC (2015) Anticarcinogenic effects of dietary phytoestrogens and their chemopreventive mechanisms. Nutr Cancer 67:796-803.

Hwang JT, Lee YK, Shin JI, and Park OJ (2009) Anti-inflammatory and anticarcinogenic effect of genistein alone or in combination with capsaicin in TPA-treated rat mammary glands or mammary cancer cell line. Ann N Y Acad Sci 1171 $415-420$.

Ibrahim NA, Shalaby AS, Farag RS, Elbaroty GS, Nofal SM, and Hassan EM (2008) Gynecological efficacy and chemical investigation of Vitex agnus-castus $L$. fruits growing in Egypt. Nat Prod Res 22:537-546.

Imai A, Ichigo S, Matsunami K, and Takagi H (2015) Premenstrual syndrome: management and pathophysiology. Clin Exp Obstet Gynecol 42:123-128.

Indran IR, Liang RL, Min TE, and Yong EL (2016) Preclinical studies and clinical evaluation of compounds from the genus Epimedium for osteoporosis and bone health. Pharmacol Ther 162:188-205.

Indran IR, Zhang SJ, Zhang ZW, Sun F, Gong Y, Wang X, Li J, Erdelmeier CA Koch E, and Yong EL (2014) Selective estrogen receptor modulator effects of epimedium extracts on breast cancer and uterine growth in nude mice. Planto Med 80:22-28.

Izadjoo M, Taavoni S, Ekbatani NN, and Haghani H (2015) The effect of valerian ora capsules on menopausal symptoms in women. Int J Rev Life Sci 5:220-226.

Izzi V, Masuelli L, Tresoldi I, Sacchetti P, Modesti A, Galvano F, and Bei R (2012) The effects of dietary flavonoids on the regulation of redox inflammatory networks. Front Biosci (Landmark Ed) 17:2396-2418.

Izzo AA, Di Carlo G, Borrelli F, and Ernst E (2005) Cardiovascular pharmacotherapy and herbal medicines: the risk of drug interaction. Int $J$ Cardiol 98:1-14.

Jacobsgaard H (2008) [The pharmacists request that pregnant women consult physicians prior to GraviFrisk use]. Ugeskr Laeger 170:867.

Jarboe CH, Schmidt CM, Nicholson JA, and Zirvi KA (1966) Uterine relaxant properties of Viburnum. Nature 212:837.

Jarboe CH, Zirvi KA, Nicholson JA, and Schmidt CM (1967) Scopoletin, an antispasmodic component of Viburnum opulus and prunifolium. J Med Chem 10 488-489.

Jarema M (2008) Herbal drug treatment. Neuroendocrinol Lett 29 (Suppl 1):93-104. Jarry H, Spengler B, Porzel A, Schmidt J, Wuttke W, and Christoffel V (2003) Evidence for estrogen receptor beta-selective activity of Vitex agnus-castus and isolated flavones. Planta Med 69:945-947.

Jefferson WN, Padilla-Banks E, Clark G, and Newbold RR (2002) Assessing estrogenic activity of phytochemicals using transcriptional activation and immature mouse uterotrophic responses. J Chromatogr B Analyt Technol Biomed Life Sci 777:179-189.

Jeong HJ, Shin YG, Kim IH, and Pezzuto JM (1999) Inhibition of aromatase activity by flavonoids. Arch Pharm Res 22:309-312. 
Jepson RG, Williams G, and Craig JC (2012) Cranberries for preventing urinary tract infections. Cochrane Database Syst Rev 10:CD001321.

Jia M, Dahlman-Wright K, and Gustafsson JA (2015) Estrogen receptor alpha and beta in health and disease. Best Pract Res Clin Endocrinol Metab 29 $557-568$.

Jiang Y, Gong P, Madak-Erdogan Z, Martin T, Jeyakumar M, Carlson K, Khan I, Smillie TJ, Chittiboyina AG, Rotte SC, et al. (2013) Mechanisms enforcing the estrogen receptor $\beta$ selectivity of botanical estrogens. FASEB J 27:4406-4418.

Jiang F, Li Y, Mu J, Hu C, Zhou M, Wang X, Si L, Ning S, and Li Z (2016) Glabridin inhibits cancer stem cell-like properties of human breast cancer cells: An epigenetic regulation of miR-148a/SMAd2 signaling. Mol Carcinog 55:929-940.

Jin Y, Qu C, Tang Y, Pang H, Liu L, Zhu Z, Shang E, Huang S, Sun D, and Duan JA (2016) Herb pairs containing Angelicae Sinensis Radix (Danggui): A review of bio-active constituents and compatibility effects. J Ethnopharmacol 181: $158-171$.

Jo EH, Hong HD, Ahn NC, Jung JW, Yang SR, Park JS, Kim SH, Lee YS, and Kang KS (2004) Modulations of the Bcl-2/Bax family were involved in the chemopreventive effects of licorice root (Glycyrrhiza uralensis Fisch) in MCF-7 human breast cancer cell. J Agric Food Chem 52:1715-1719.

Jo EH, Kim SH, Ra JC, Kim SR, Cho SD, Jung JW, Yang SR, Park JS, Hwang JW, Aruoma OI, et al. (2005) Chemopreventive properties of the ethanol extract of chinese licorice (Glycyrrhiza uralensis) root: induction of apoptosis and G1 cell cycle arrest in MCF-7 human breast cancer cells. Cancer Lett 230:239-247.

$\mathrm{Ju}$ YH, Allred KF, Allred CD, and Helferich WG (2006) Genistein stimulates growth of human breast cancer cells in a novel, postmenopausal animal model, with low plasma estradiol concentrations. Carcinogenesis 27:1292-1299.

Junio HA, Sy-Cordero AA, Ettefagh KA, Burns JT, Micko KT, Graf TN, Richter SJ, Cannon RE, Oberlies NH, and Cech NB (2011) Synergy-directed fractionation of botanical medicines: a case study with goldenseal (Hydrastis canadensis). J Nat Prod 74:1621-1629.

Kamel M, Shouman S, El-Merzebany M, Kilic G, Veenstra T, Saeed M, Wagih M, Diaz-Arrastia C, Patel D, and Salama S (2012) Effect of tumour necrosis factoralpha on estrogen metabolic pathways in breast cancer cells. J Cancer 3: 310-321.

Kang MR, Park KH, Oh SJ, Yun J, Lee CW, Lee MY, Han SB, and Kang JS (2015) Cardiovascular protective effect of glabridin: Implications in LDL oxidation and inflammation. Int Immunopharmacol 29:914-918.

Kao TC, Wu CH, and Yen GC (2014) Bioactivity and potential health benefits of licorice. J Agric Food Chem 62:542-553.

Kashani L, Saedi N, and Akhondzadeh S (2010) Femicomfort in the treatment of premenstrual syndromes: a double-blind, randomized and placebo controlled trial. Iran $J$ Psychiatry 5:47-50.

Kashefi F, Khajehei M, Alavinia M, Golmakani E, and Asili J (2015) Effect of ginger (Zingiber officinale) on heavy menstrual bleeding: a placebo-controlled, randomized clinical trial. Phytother Res 29:114-119.

Kashefi F, Khajehei M, Tabatabaeichehr M, Alavinia M, and Asili J (2014) Comparison of the effect of ginger and zinc sulfate on primary dysmenorrhea: a placebocontrolled randomized trial. Pain Manag Nurs 15:826-833.

Kaspar JW and Jaiswal AK (2010) Antioxidant-induced phosphorylation of tyrosine 486 leads to rapid nuclear export of Bach1 that allows Nrf2 to bind to the antioxidant response element and activate defensive gene expression. J Biol Chem $\mathbf{2 8 5}$ 153-162.

Kaszkin-Bettag M, Beck S, Richardson A, Heger PW, and Beer AM (2008) Efficacy of the special extract ERr 731 from rhapontic rhubarb for menopausal complaints: a 6-month open observational study. Altern Ther Health Med 14:32-38.

Kelber O, Nieber K, and Kraft K (2014) Valerian: no evidence for clinically relevant interactions. Evid Based Complement Alternat Med 2014:879396.

Kelderhouse K and Taylor JS (2013) A review of treatment and management modalities for premenstrual dysphoric disorder. Nurs Womens Health 17:294-305.

Kennedy DA, Lupattelli A, Koren G, and Nordeng H (2013) Herbal medicine use in pregnancy: results of a multinational study. BMC Complement Altern Med 13: 355

Kennedy D and Seely D (2010) Clinically based evidence of drug-herb interactions: a systematic review. Expert Opin Drug Saf 9:79-124.

Khan SA, Chatterton RT, Michel N, Bryk M, Lee O, Ivancic D, Heinz R, Zalles CM, Helenowski IB, Jovanovic BD, et al. (2012b) Soy isoflavone supplementation for breast cancer risk reduction: a randomized phase II trial. Cancer Prev Res (Phila) 5:309-319

Khan AQ, Khan R, Rehman MU, Lateef A, Tahir M, Ali F, and Sultana S (2012a) Soy isoflavones (daidzein \& genistein) inhibit 12-O-tetradecanoylphorbol-13-acetate (TPA)-induced cutaneous inflammation via modulation of COX-2 and NF-кB in Swiss albino mice. Toxicology 302:266-274.

Kim IG, Kang SC, Kim KC, Choung ES, and Zee OP (2008) Screening of estrogenic and antiestrogenic activities from medicinal plants. Environ Toxicol Pharmacol 25: $75-82$.

Kim S, Kim SH, Hur SM, Lee SK, Kim WW, Kim JS, Kim JH, Choe JH, Nam SJ, Lee JE, et al. (2009) Silibinin prevents TPA-induced MMP-9 expression by downregulation of COX-2 in human breast cancer cells. $J$ Ethnopharmacol 126: $252-257$

Kim J, Kim J, Shim J, Lee S, Kim J, Lim SS, Lee KW, and Lee HJ (2013b) Licoricederived dehydroglyasperin C increases MKP-1 expression and suppresses inflammation-mediated neurodegeneration. Neurochem Int 63:732-740.

Kim DH, Park EK, Bae EA, and Han MJ (2000) Metabolism of rhaponticin and chrysophanol 8-o-beta-D-glucopyranoside from the rhizome of rheum undulatum by human intestinal bacteria and their anti-allergic actions. Biol Pharm Bull 23: 830-833.

Kim BR, Seo HS, Ku JM, Kim GJ, Jeon CY, Park JH, Jang BH, Park SJ, Shin YC, and Ko SG (2013a) Silibinin inhibits the production of pro-inflammatory cytokines through inhibition of NF-кB signaling pathway in HMC-1 human mast cells. Inflamm Res 62:941-950.
Kim E, Sy-Cordero A, Graf TN, Brantley SJ, Paine MF, and Oberlies NH (2011) Isolation and identification of intestinal CYP3A inhibitors from cranberry (Vaccinium macrocarpon) using human intestinal microsomes. Planta Med 77:265-270.

Kintscher U (2012) Reuptake inhibitors of dopamine, noradrenaline, and serotonin. Handbook Exp Pharmacol 209:339-347.

Kiruthiga PV, Karthikeyan K, Archunan G, Pandian SK, and Devi KP (2015) Silymarin prevents benzo(a)pyrene-induced toxicity in Wistar rats by modulating xenobiotic-metabolizing enzymes. Toxicol Ind Health 31:523-541.

Köhle C and Bock KW (2007) Coordinate regulation of Phase I and II xenobiotic metabolisms by the Ah receptor and Nrf2. Biochem Pharmacol 73: 1853-1862.

Kole L, Giri B, Manna SK, Pal B, and Ghosh S (2011) Biochanin-A, an isoflavon, showed anti-proliferative and anti-inflammatory activities through the inhibition of iNOS expression, p38-MAPK and ATF-2 phosphorylation and blocking $\mathrm{NF}_{\kappa} \mathrm{B}$ nuclear translocation. Eur J Pharmacol 653:8-15.

Komesaroff PA, Black CV, Cable V, and Sudhir K (2001) Effects of wild yam extract on menopausal symptoms, lipids and sex hormones in healthy menopausal women. Climacteric 4:144-150.

Kondo K, Shiba M, Nakamura R, Morota T, and Shoyama Y (2007a) Constituent properties of licorices derived from Glycyrrhiza uralensis, G. glabra, or G. inflata identified by genetic information. Biol Pharm Bull 30:1271-1277.

Kondo K, Shiba M, Yamaji H, Morota T, Zhengmin C, Huixia P, and Shoyama Y (2007b) Species identification of licorice using nrDNA and cpDNA genetic markers. Biol Pharm Bull 30:1497-1502.

Krajka-Kuźniak V, Paluszczak J, and Baer-Dubowska W (2013) Xanthohumol induces phase II enzymes via Nrf2 in human hepatocytes in vitro. Toxicol In Vitro 27: 149-156.

Kumar A, Goyal R, Kumar S, Jain S, Jain N, and Kumar P (2015) Estrogenic and anti-Alzheimer's studies of Zingiber officinalis as well as Amomum subulatum Roxb.: the success story of dry techniques. Med Chem Res 24:1089-1097.

Lacey JV Jr (2014) The WHI ten year's later: an epidemiologist's view. J Steroid Biochem Mol Biol 142:12-15.

Lagari VS and Levis S (2014) Phytoestrogens for menopausal bone loss and climacteric symptoms. J Steroid Biochem Mol Biol 139:294-301.

Lai JN, Wu CT, and Wang JD (2012) Prescription pattern of chinese herbal products for breast cancer in taiwan: a population-based study. Evid Based Complement Alternat Med 2012:891893.

Lam AN, Demasi M, James MJ, Husband AJ, and Walker C (2004) Effect of red clover isoflavones on cox-2 activity in murine and human monocyte/macrophage cells. Nutr Cancer 49:89-93.

Lampe JW, Nishino Y, Ray RM, Wu C, Li W, Lin MG, Gao DL, Hu Y, Shannon J, Stalsberg H, et al. (2007) Plasma isoflavones and fibrocystic breast conditions and breast cancer among women in Shanghai, China. Cancer Epidemiol Biomarkers Prev 16:2579-2586.

Lau CB, Ho TC, Chan TW, and Kim SC (2005) Use of dong quai (Angelica sinensis) to treat peri- or postmenopausal symptoms in women with breast cancer: is it appropriate? Menopause 12:734-740.

Lau GT, Ye L, and Leung LK (2010) The licorice flavonoid isoliquiritigenin suppresses phorbol ester-induced cyclooxygenase-2 expression in the non-tumorigenic MCF-10A breast cell line. Planta Med 76:780-785.

Lee PG, Kim J, Kim EJ, Jung E, Pandey BP, and Kim BG (2016) P212A mutant of dihydrodaidzein reductase enhances (S)-equol productivity and enantioselectivity in recombinant Escherichia coli whole cell reaction system. Appl Environ Microbiol 22:1992-2002.

Lee IS, Lim J, Gal J, Kang JC, Kim HJ, Kang BY, and Choi HJ (2011) Antiinflammatory activity of xanthohumol involves heme oxygenase-1 induction via NRF2-ARE signaling in microglial BV2 cells. Neurochem Int 58:153-160.

Lee NM and Saha S (2011) Nausea and vomiting of pregnancy. Gastroenterol Clin North Am 40:309-334, vii.

Lee MS, Shin BC, Yang EJ, Lim HJ, and Ernst E (2011c) Maca (Lepidium meyenii) for treatment of menopausal symptoms: A systematic review. Maturitas $\mathbf{7 0}$ $227-233$

Lethaby A, Marjoribanks J, Kronenberg F, Roberts H, Eden J, and Brown J (2013) Phytoestrogens for menopausal vasomotor symptoms. Cochrane Database Syst Rev 12:CD001395.

Leung HY, Yung LH, Poon CH, Shi G, Lu AL, and Leung LK (2009) Genistein protects against polycyclic aromatic hydrocarbon-induced oxidative DNA damage in non-cancerous breast cells MCF-10A. Br J Nutr 101:257-262.

L'Héritier F, Marques M, Fauteux M, and Gaudreau L (2014) Defining molecular sensors to assess long-term effects of pesticides on carcinogenesis. Int J Mol Sci $\mathbf{1 5}$ : 17148-17161.

Li Y, Chen H, Hardy TM, and Tollefsbol TO (2013a) Epigenetic regulation of multiple tumor-related genes leads to suppression of breast tumorigenesis by dietary genistein. PLoS One 8:e54369.

Li J, Hua Y, Ji P, Yao W, Zhao H, Zhong L, and Wei Y (2016) Effects of volatile oils of Angelica sinensis on an acute inflammation rat model. Pharm Biol DOI: 10.3109/ 13880209.2015.1133660 [published ahead of print].

Li Y, Meeran SM, Patel SN, Chen H, Hardy TM, and Tollefsbol TO (2013c) Epigenetic reactivation of estrogen receptor- $\alpha(\mathrm{ER} \alpha)$ by genistein enhances hormonal therapy sensitivity in ER $\alpha$-negative breast cancer. Mol Cancer 12:9 10.1186/1476-45981112-1189.

Li LH, Wu LJ, Tashiro SI, Onodera S, Uchiumi F, and Ikejima T (2007) Activation of the SIRT1 pathway and modulation of the cell cycle were involved in silymarin's protection against UV-induced A375-S2 cell apoptosis. J Asian Nat Prod Res 9: $245-252$.

Li S, Zhang B, and Zhang N (2011) Network target for screening synergistic drug combinations with application to traditional Chinese medicine. BMC Syst Biol 5 (Suppl 1):S10.

Li Y, Zhao H, Wang Y, Zheng H, Yu W, Chai H, Zhang J, Falck JR, Guo AM, Yue J, et al. (2013b) Isoliquiritigenin induces growth inhibition and apoptosis through 
downregulating arachidonic acid metabolic network and the deactivation of PI3K/ Akt in human breast cancer. Toxicol Appl Pharmacol 272:37-48.

Lin F and Giusti MM (2005) Effects of solvent polarity and acidity on the extraction efficiency of isoflavones from soybeans (Glycine max). J Agric Food Chem 53: 3795-3800.

Lindstrom A, Ooyen C, Lynch ME, Blumenthal M, and Kawa K (2014) Sales of herbal dietary supplements increase by $7.9 \%$ in 2013 , marking a decade of rising sales: turmeric supplements climb to top ranking in natural channel. HerbalGram 103: $52-56$.

Little P, Moore MV, Turner S, Rumsby K, Warner G, Lowes JA, Smith H, Hawke C Leydon G, Arscott A, et al. (2010) Effectiveness of five different approaches in management of urinary tract infection: randomised controlled trial. BMJ 340: c199.

Liu J, Burdette JE, Sun Y, Deng S, Schlecht SM, Zheng W, Nikolic D, Mahady G, van Breemen RB, Fong $\mathrm{HH}$, et al. (2004) Isolation of linoleic acid as an estrogenic compound from the fruits of Vitex agnus-castus L. (chaste-berry). Phytomedicine 11:18-23.

Liu J, Burdette JE, Xu H, Gu C, van Breemen RB, Bhat KP, Booth N, Constantinou AI, Pezzuto JM, Fong HH, et al. (2001) Evaluation of estrogenic activity of plant extracts for the potential treatment of menopausal symptoms. J Agric Food Chem 49:2472-2479.

Liu G, Eggler AL, Dietz BM, Mesecar AD, Bolton JL, Pezzuto JM, and van Breemen $\mathrm{RB}$ (2005) Screening method for the discovery of potential cancer chemoprevention agents based on mass spectrometric detection of alkylated Keap1. Anal Chem $\mathbf{7 7}$ $6407-6414$

Liu H, Wang J, Zhou W, Wang Y, and Yang L (2013) Systems approaches and polypharmacology for drug discovery from herbal medicines: an example using licorice. $J$ Ethnopharmacol 146:773-793.

Lloyd KB and Hornsby LB (2009) Complementary and alternative medications for women's health issues. Nutr Clin Pract 24:589-608.

López-Gutiérrez N, Romero-González R, Garrido Frenich A, and Martínez Vidal JL (2014) Identification and quantification of the main isoflavones and other phytochemicals in soy based nutraceutical products by liquid chromatography-orbitrap high resolution mass spectrometry. J Chromatogr A 1348:125-136.

Lorusso V and Marech I (2013) Novel plant-derived target drugs: a step forward from licorice? Expert Opin Ther Targets 17:333-335.

Low Dog T (2005) Menopause: a review of botanical dietary supplements. Am J Med 118 (Suppl 12B):98-108

Lowenthal J and Birnbaum H (1969) Vitamin K and coumarin anticoagulants: dependence of anticoagulant effect on inhibition of vitamin K transport. Science $\mathbf{1 6 4}$ 181-183.

Lupinacci E, Meijerink J, Vincken JP, Gabriele B, Gruppen H, and Witkamp RF (2009) Xanthohumol from hop (Humulus lupulus L.) is an efficient inhibitor of monocyte chemoattractant protein-1 and tumor necrosis factor-alpha release in LPS-stimulated RAW 264.7 mouse macrophages and U937 human monocytes. $J$ Agric Food Chem 57:7274-7281.

Ma H, He X, Yang Y, Li M, Hao D, and Jia Z (2011) The genus Epimedium: an ethnopharmacological and phytochemical review. $J$ Ethnopharmacol 134: $519-541$.

Ma L, Lin S, Chen R, Zhang Y, Chen F, and Wang X (2010) Evaluating therapeutic effect in symptoms of moderate-to-severe premenstrual syndrome with Vitex agnus castus (BNO 1095) in Chinese women. Aust N Z J Obstet Gynaecol 50:189-193.

Maggiolini M, Statti G, Vivacqua A, Gabriele S, Rago V, Loizzo M, Menichini F, and Amdò S (2002) Estrogenic and antiproliferative activities of isoliquiritigenin in MCF7 breast cancer cells. J Steroid Biochem Mol Biol 82:315-322.

Mahady GB, Low Dog T, Barrett ML, Chavez ML, Gardiner P, Ko R, Marles RJ, Pellicore LS, Giancaspro GI, and Sarma DN (2008) United States Pharmacopeia review of the black cohosh case reports of hepatotoxicity. Menopause 15:628-638.

Malewicz B, Wang Z, Jiang C, Guo J, Cleary MP, Grande JP, and Lü J (2006) Enhancement of mammary carcinogenesis in two rodent models by silymarin dietary supplements. Carcinogenesis 27:1739-1747.

Manna SK, Mukhopadhyay A, Van NT, and Aggarwal BB (1999) Silymarin suppresses TNF-induced activation of NF-kappa B, c-Jun N-terminal kinase, and apoptosis. J Immunol 163:6800-6809.

Manson J and Branch H (2014) The women's health initiative: the latest findings from long-term follow-up. Womens Health (Lond Engl) 10:125-128.

Marcellin L and Chantry AA (2015) [Breastfeeding (part III): Breastfeeding complications-Guidelines for clinical practice]. J Gynecol Obstet Biol Reprod (Paris) 44 $1084-1090$

Marini H, Minutoli L, Polito F, Bitto A, Altavilla D, Atteritano M, Gaudio A, Mazzaferro S, Frisina A, Frisina N, et al. (2007) Effects of the phytoestrogen genistein on bone metabolism in osteopenic postmenopausal women: a randomized trial. Ann Intern Med 146:839-847.

Marjoribanks J, Brown J, O'Brien PM, and Wyatt K (2013) Selective serotonin reuptake inhibitors for premenstrual syndrome. Cochrane Database Syst Rev 6: CD001396.

Marx W, Kiss N, and Isenring L (2015) Is ginger beneficial for nausea and vomiting? An update of the literature. Curr Opin Support Palliat Care 9:189-195.

Masilamani M, Wei J, and Sampson HA (2012) Regulation of the immune response by soybean isoflavones. Immunol Res 54:95-110.

Maskarinec G, Steude JS, Franke AA, and Cooney RV (2009) Inflammatory markers in a 2-year soy intervention among premenopausal women. $J$ Inflamm (Lond) 6:9

Mason JK and Thompson LU (2014) Flaxseed and its lignan and oil components: can they play a role in reducing the risk of and improving the treatment of breast cancer? Appl Physiol Nutr Metab 39:663-678.

Medjakovic S and Jungbauer A (2008) Red clover isoflavones biochanin A and formononetin are potent ligands of the human aryl hydrocarbon receptor. $J$ Steroid Biochem Mol Biol 108:171-177.

Meier B, Berger D, Hoberg E, Sticher O, and Schaffner W (2000) Pharmacological activities of Vitex agnus-castus extracts in vitro. Phytomedicine 7:373-381.
Menati L, Khaleghinezhad K, Tadayon M, and Siahpoosh A (2014) Evaluation of contextual and demographic factors on licorice effects on reducing hot flashes in postmenopause women. Health Care Women Int 35:87-99.

Mense SM, Hei TK, Ganju RK, and Bhat HK (2008) Phytoestrogens and breast cancer prevention: possible mechanisms of action. Environ Health Perspect 116: 426-433.

Mersereau JE, Levy N, Staub RE, Baggett S, Zogovic T, Chow S, Ricke WA, Tagliaferri M, Cohen I, Bjeldanes LF, et al. (2008) Liquiritigenin is a plant-derived highly selective estrogen receptor beta agonist. Mol Cell Endocrinol 283:49-57.

Messina M, Ho S, and Alekel DL (2004) Skeletal benefits of soy isoflavones: a review of the clinical trial and epidemiologic data. Curr Opin Clin Nutr Metab Care 7: $649-658$

Micklefield GH, Redeker Y, Meister V, Jung O, Greving I, and May B (1999) Effects of ginger on gastroduodenal motility. Int J Clin Pharmacol Ther 37:341-346.

Milić N, Milosević N, Suvajdzić L, Zarkov M, and Abenavoli L (2013) New therapeutic potentials of milk thistle (Silybum marianum). Nat Prod Commun 8:1801-1810.

Milligan SR, Kalita JC, Heyerick A, Rong H, De Cooman L, and De Keukeleire D (1999) Identification of a potent phytoestrogen in hops (Humulus lupulus L.) and beer. J Clin Endocrinol Metab 84:2249-2252.

Mirabi P, Dolatian M, Mojab F, and Majd HA (2011) Effects of valerian on the severity and systemic manifestations of dysmenorrhea. Int J Gynaecol Obstet 115 $285-288$.

Mirabi P and Mojab F (2013) The effects of valerian root on hot flashes in menopausal women. Iran J Pharm Res 12:217-222.

Mirza S, Sharma G, Parshad R, Gupta SD, Pandya P, and Ralhan R (2013) Expression of DNA methyltransferases in breast cancer patients and to analyze the effect of natural compounds on DNA methyltransferases and associated proteins. $J$ Breast Cancer 16:23-31.

Möller F, Zierau O, Jandausch A, Rettenberger R, Kaszkin-Bettag M, and Vollmer G (2007) Subtype-specific activation of estrogen receptors by a special extract of Rheum rhaponticum (ERr 731), its aglycones and structurally related compounds in U2OS human osteosarcoma cells. Phytomedicine 14:716-726.

Monteiro R, Calhau C, Silva AO, Pinheiro-Silva S, Guerreiro S, Gärtner F, Azevedo I, and Soares R (2008) Xanthohumol inhibits inflammatory factor production and angiogenesis in breast cancer xenografts. J Cell Biochem 104:1699-1707.

Monteiro R, Faria A, Azevedo I, and Calhau C (2007) Modulation of breast cancer cell survival by aromatase inhibiting hop (Humulus lupulus L.) flavonoids. J Steroid Biochem Mol Biol 105:124-130.

Montserrat-de la Paz S, Fernandez-Arche MA, Angel-Martin M, and Garcia-Gimenez MD (2014) Phytochemical characterization of potential nutraceutical ingredients from Evening Primrose oil (Oenothera biennis L.). Phytochem Lett 8:158-162.

Morabito N, Crisafulli A, Vergara C, Gaudio A, Lasco A, Frisina N, D'Anna R, Corrado F, Pizzoleo MA, Cincotta M, et al. (2002) Effects of genistein and hormonereplacement therapy on bone loss in early postmenopausal women: a randomized double-blind placebo-controlled study. J Bone Miner Res 17:1904-1912.

Morimoto Y, Maskarinec G, Park SY, Ettienne R, Matsuno RK, Long C, Steffen AD, Henderson BE, Kolonel LN, Le Marchand L, et al. (2014) Dietary isoflavone intake is not statistically significantly associated with breast cancer risk in the Multiethnic Cohort. Br J Nutr 112:976-983.

Morris E and Currie H (2013) Estrogen deficiency: education for all! Menopause Int 19:55.

Mortel M and Mehta SD (2013) Systematic review of the efficacy of herbal galactogogues. J Hum Lact 29:154-162.

Mrozikiewicz PM, Bogacz A, Czerny B, Karasiewicz M, Kujawski R, Mikolajczak PL Seremak-Mrozikiewicz A, Grzeskowiak E, and Bobkiewicz-Kozlowska T (2010) The influence of a standardized soybean extract (Glycine max) on the expression level of cytochrome P450 genes in vivo. Ginekol Pol 81:516-520.

Mu J, Ning SL, Wang XX, Si L, Jiang F, Li Y, and Li Z (2015) The repressive effect of miR-520a on NF-kappa B/IL-6/STAT-3 signal involved in the glabridin-induced anti-angiogenesis in human breast cancer cells. Rsc Adv 5:34257-34264.

Mueller M, Hobiger S, and Jungbauer A (2010) Red clover extract: a source for substances that activate peroxisome proliferator-activated receptor alpha and ameliorate the cytokine secretion profile of lipopolysaccharide-stimulated macrophages. Menopause 17:379-387.

Murray IA, Patterson AD, and Perdew GH (2014) Aryl hydrocarbon receptor ligands in cancer: friend and foe. Nat Rev Cancer 14:801-814.

Nachtigall LE (2010) Therapy: nonhormonal treatment of hot flashes-a viable alternative? Nat Rev Endocrinol 6:66-67.

Nagaraju GP, Zafar SF, and El-Rayes BF (2013) Pleiotropic effects of genistein in metabolic, inflammatory, and malignant diseases. Nutr Rev 71:562-572.

Nahidi F, Zare E, Mojab F, and Alavi-Majd H (2012) Effects of licorice on relief and recurrence of menopausal hot flashes. Iran J Pharm Res 11:541-548.

Nakamura K, Nishihata T, Jin JS, Ma CM, Komatsu K, Iwashima M, and Hattori M (2011) The C-glucosyl bond of puerarin was cleaved hydrolytically by a human intestinal bacterium strain PUE to yield its aglycone daidzein and an intact glucose. Chem Pharm Bull (Tokyo) 59:23-27.

Nedrow A, Miller J, Walker M, Nygren P, Huffman LH, and Nelson HD (2006) Complementary and alternative therapies for the management of menopauserelated symptoms: a systematic evidence review. Arch Intern Med 166 $1453-1465$

Nelson HD (2008) Menopause. Lancet 371:760-770.

Nelson HD, Vesco KK, Haney E, Fu R, Nedrow A, Miller J, Nicolaidis C, Walker M, and Humphrey L (2006) Nonhormonal therapies for menopausal hot flashes: systematic review and meta-analysis. JAMA 295:2057-2071.

Nicholson JA, Darby TD, and Jarboe CH (1972) Viopudial, a hypotensive and smooth muscle antispasmodic from Viburnum opulus. Proc Soc Exp Biol Med 140:457-461.

Nickel JC (2007) Urinary tract infections and resistant bacteria: Highlights of a symposium at the Combined Meeting of the 25th International Congress of Chemotherapy (ICC) and the 17th European Congress of Clinical Microbiology and 
Infectious Diseases (ECCMID), March 31-April 3, 2007, Munich, Germany. Rev Urol 9:78-80.

Nierode G, Kwon PS, Dordick JS, and Kwon SJ (2016) Cell-based assay design for high-content screening of drug candidates. J Microbiol Biotechnol 26:213-225.

Nieves JW (2013) Skeletal effects of nutrients and nutraceuticals, beyond calcium and vitamin D. Osteoporos Int 24:771-786.

Nikolic D, Li Y, Chadwick LR, Pauli GF, and van Breemen RB (2005) Metabolism of xanthohumol and isoxanthohumol, prenylated flavonoids from hops (Humulus lupulus L.), by human liver microsomes. J Mass Spectrom 40:289-299.

Nilsson S, Mäkelä S, Treuter E, Tujague M, Thomsen J, Andersson G, Enmark E, Pettersson K, Warner M, and Gustafsson JA (2001) Mechanisms of estrogen action. Physiol Rev 81:1535-1565.

North American Menopause Society (2004) Treatment of menopause-associated vasomotor symptoms: position statement of The North American Menopause Society. Menopause 11:11-33.

North American Menopause Society (2011) The role of soy isoflavones in menopausal health: report of The North American Menopause Society/Wulf H. Utian Translational Science Symposium in Chicago, IL (October 2010). Menopause 18: $732-753$.

Ntie-Kang F, Lifongo LL, Mbaze LM, Ekwelle N, Owono Owono LC, Megnassan E, Judson PN, Sippl W, and Efange SM (2013) Cameroonian medicinal plants: a bioactivity versus ethnobotanical survey and chemotaxonomic classification. BMC Complement Altern Med 13:147.

Obeid E, Nanda R, Fu YX, and Olopade OI (2013) The role of tumor-associated macrophages in breast cancer progression (review). review Int $J$ Oncol 43: $5-12$.

Occhiuto F, Pino A, Palumbo DR, Samperi S, De Pasquale R, Sturlese E, and Circosta C (2009) Relaxing effects of Valeriana officinalis extracts on isolated human nonpregnant uterine muscle. $J$ Pharm Pharmacol 61:251-256.

Oh YC, Jeong YH, Cho WK, Ha JH, Lee SJ, and Ma JY (2015) Inhibitory effects of epimedium herb on the inflammatory response in vitro and in vivo. Am J Chin Med 43:953-968.

Overk CR, Guo J, Chadwick LR, Lantvit DD, Minassi A, Appendino G, Chen SN, Lankin DC, Farnsworth NR, Pauli GF, et al. (2008) In vivo estrogenic comparisons of Trifolium pratense (red clover) Humulus lupulus (hops), and the pure compounds isoxanthohumol and 8-prenylnaringenin. Chem Biol Interact 176:30-39.

Overk CR, Peng KW, Asghodom RT, Kastrati I, Lantvit DD, Qin Z, Frasor J, Bolton JL, and Thatcher GR (2007) Structure-activity relationships for a family of benzothiophene selective estrogen receptor modulators including raloxifene and arzoxifene. ChemMedChem 2:1520-1526.

Overk CR, Yao P, Chadwick LR, Nikolic D, Sun Y, Cuendet MA, Deng Y, Hedayat AS, Pauli GF, Farnsworth NR, et al. (2005) Comparison of the in vitro estrogenic activities of compounds from hops (Humulus lupulus) and red clover (Trifolium pratense). J Agric Food Chem 53:6246-6253.

Ozgoli G, Goli M, and Moattar F (2009) Comparison of effects of ginger, mefenamic acid, and ibuprofen on pain in women with primary dysmenorrhea. J Altern Complement Med 15:129-132.

Park KS, Park KI, Hwang DS, Lee JM, Jang JB, and Lee CH (2014) A review of in vitro and in vivo studies on the efficacy of herbal medicines for primary dysmenorrhea. Evid Based Complement Alternat Med 2014:296860.

Park EK, Shin J, Bae EA, Lee YC, and Kim DH (2006) Intestinal bacteria activate estrogenic effect of main constituents puerarin and daidzin of Pueraria thunbergiana. Biol Pharm Bull 29:2432-2435.

Pasternak B, Svanström H, Mølgaard-Nielsen D, Melbye M, and Hviid A (2013) Metoclopramide in pregnancy and risk of major congenital malformations and fetal death. JAMA 310:1601-1611.

Patocka J and Jaki J (2010) Biomedically relevant chemical constituents of Valeriana officinalis. J Appl Biomed 8:11-18.

Pauli GF, Chen SN, Simmler C, Lankin DC, Gödecke T, Jaki BU, Friesen JB, McAlpine JB, and Napolitano JG (2014) Importance of purity evaluation and the potential of quantitative ${ }^{1} \mathrm{H}$ NMR as a purity assay. J Med Chem 57:9220-9231.

Pelkonen O, Xu Q, and Fan TP (2014) Why is research on herbal medicinal products important and how can we improve its quality? J Tradit Complement Med 4:1-7.

Peng F, Du Q, Peng C, Wang N, Tang H, Xie X, Shen J, and Chen J (2015) A Review: The pharmacology of isoliquiritigenin. Phytother Res 29:969-977.

Pertz HH, Lehmann J, Roth-Ehrang R, and Elz S (2011) Effects of ginger constituents on the gastrointestinal tract: role of cholinergic M3 and serotonergic 5-HT3 and 5-HT4 receptors. Planta Med 77:973-978.

Pferschy-Wenzig EM and Bauer R (2015) The relevance of pharmacognosy in pharmacological research on herbal medicinal products. Epilepsy Behav 52 (Pt B): 344-362.

Piersen CE (2003) Phytoestrogens in botanical dietary supplements: implications for cancer. Integr Cancer Ther 2:120-138.

Piersen CE, Booth NL, Sun Y, Liang W, Burdette JE, van Breemen RB, Geller SE, Gu C, Banuvar S, Shulman LP, et al. (2004) Chemical and biological characterization and clinical evaluation of botanical dietary supplements: a phase I red clover extract as a model. Curr Med Chem 11:1361-1374.

Piotrowska H, Kucinska M, and Murias M (2012) Biological activity of piceatannol: leaving the shadow of resveratrol. Mutat Res 750:60-82.

Plazar J, Zegura B, Lah TT, and Filipic M (2007) Protective effects of xanthohumol against the genotoxicity of benzo(a)pyrene (BaP), 2-amino-3-methylimidazo[4,5f] quinoline (IQ) and tert-butyl hydroperoxide (t-BOOH) in HepG2 human hepatoma cells. Mutat Res 632:1-8.

Plísková M, Vondrácek J, Kren V, Gazák R, Sedmera P, Walterová D, Psotová J, Simánek V, and Machala M (2005) Effects of silymarin flavonolignans and synthetic silybin derivatives on estrogen and aryl hydrocarbon receptor activation Toxicology 215:80-89.

Poluzzi E, Piccinni C, Raschi E, Rampa A, Recanatini M, and De Ponti F (2014) Phytoestrogens in postmenopause: the state of the art from a chemical, pharmacological and regulatory perspective. Curr Med Chem 21:417-436.
Polyak SJ, Morishima C, Lohmann V, Pal S, Lee DY, Liu Y, Graf TN, and Oberlies $\mathrm{NH}$ (2010) Identification of hepatoprotective flavonolignans from silymarin. Proc Natl Acad Sci USA 107:5995-5999.

Pons DG, Nadal-Serrano M, Torrens-Mas M, Oliver J, and Roca P (2016) The phytoestrogen genistein affects breast cancer cells treatment depending on the ER $\alpha /$ ERß ratio. J Cell Biochem 117:218-229.

Possemiers S, Bolca S, Grootaert C, Heyerick A, Decroos K, Dhooge W, De Keukeleire D, Rabot S, Verstraete W, and Van de Wiele T (2006) The prenylflavonoid isoxanthohumol from hops (Humulus lupulus L.) is activated into the potent phytoestrogen 8-prenylnaringenin in vitro and in the human intestine. J Nutr 136:1862-1867.

Powell SL, Gödecke T, Nikolic D, Chen SN, Ahn S, Dietz B, Farnsworth NR, van Breemen RB, Lankin DC, Pauli GF, et al. (2008) In vitro serotonergic activity of black cohosh and identification of N(omega)-methylserotonin as a potential active constituent. J Agric Food Chem 56:11718-11726.

Powers CN and Setzer WN (2015) A molecular docking study of phytochemical estrogen mimics from dietary herbal supplements. In Silico Pharmacol 3:4.

Qiu F, McAlpine JB, Krause EC, Chen S-N, and Pauli GF (2014) Pharmacognosy of Black Cohosh: The Phytochemical and Biological Profile of a Major Botanical Dietary Supplement, in Progress in the Chemistry of Organic Natural Products (book 99) (Kinghorn DA, Falk H, and Kobayashi J, eds) pp 1-68, Springer, NY.

Raftogianis R, Creveling C, Weinshilboum R, and Weisz J (2000) Estrogen metabolism by conjugation. J Natl Cancer Inst Monogr 27:113-124.

Rahman I, Biswas SK, and Kirkham PA (2006) Regulation of inflammation and redox signaling by dietary polyphenols. Biochem Pharmacol 72:1439-1452.

Rajnarayana K, Reddy MS, Vidyasagar J, and Krishna DR (2004) Study on the influence of silymarin pretreatment on metabolism and disposition of metronidazole. Arzneimittelforschung 54:109-113.

Reinwald S and Weaver CM (2006) Soy isoflavones and bone health: a double-edged sword? J Nat Prod 69:450-459.

Ren SY and Xu X (2015) Role of autophagy in metabolic syndrome-associated heart disease. Biochim Biophys Acta 1852:225-231.

Resende FA, de Oliveira AP, de Camargo MS, Vilegas W, and Varanda EA (2013) Evaluation of estrogenic potential of flavonoids using a recombinant yeast strain and MCF7/BUS cell proliferation assay. PLoS One 8:e74881.

Rice-Evans C (2001) Flavonoid antioxidants. Curr Med Chem 8:797-807.

Roberts DW, Doerge DR, Churchwell MI, Gamboa da Costa G, Marques MM, and Tolleson WH (2004) Inhibition of extrahepatic human cytochromes P450 1A1 and $1 \mathrm{~B} 1$ by metabolism of isoflavones found in Trifolium pratense (red clover). J Agric Food Chem 52:6623-6632.

Rodríguez-Morató J, Farré M, Pérez-Mañá C, Papaseit E, Martínez-Riera R, de la Torre R, and Pizarro N (2015) Pharmacokinetic comparison of soy isoflavone extracts in human plasma. J Agric Food Chem 63:6946-6953.

Rossi R, Porta S, and Canovi B (2010) Overview on cranberry and urinary tract infections in females. J Clin Gastroenterol 44 (Suppl 1):S61-S62.

Rossouw JE, Anderson GL, Prentice RL, LaCroix AZ, Kooperberg C, Stefanick ML, Jackson RD, Beresford SA, Howard BV, Johnson KC, et al.; Writing Group for the Women's Health Initiative Investigators (2002) Risks and benefits of estrogen plus progestin in healthy postmenopausal women: principal results From the Women's Health Initiative randomized controlled trial. JAMA 288:321-333.

Rowe H, Baker T, and Hale TW (2013) Maternal medication, drug use, and breastfeeding. Pediatr Clin North Am 60:275-294.

Ruhlen RL, Haubner J, Tracy JK, Zhu W, Ehya H, Lamberson WR, Rottinghaus GE, and Sauter ER (2007) Black cohosh does not exert an estrogenic effect on the breast. Nutr Cancer 59:269-277.

Russo M, Russo GL, Daglia M, Kasi PD, Ravi S, Nabavi SF, and Nabavi SM (2016) Understanding genistein in cancer: The "good" and the "bad" effects: A review. Food Chem 196:589-600.

Sahin K, Tuzcu M, Sahin N, Akdemir F, Ozercan I, Bayraktar S, and Kucuk O (2011) Inhibitory effects of combination of lycopene and genistein on 7,12- dimethyl benz(a)anthracene-induced breast cancer in rats. Nutr Cancer 63:1279-1286.

Sakla MS, Shenouda NS, Ansell PJ, Macdonald RS, and Lubahn DB (2007) Genistein affects HER2 protein concentration, activation, and promoter regulation in BT-474 human breast cancer cells. Endocrine 32:69-78.

Salter S and Brownie S (2010) Treating primary insomnia - the efficacy of valerian and hops. Aust Fam Physician 39:433-437.

Samadi AK, Bilsland A, Georgakilas AG, Amedei A, Amin A, Bishayee A, Azmi AS, Lokeshwar BL, Grue B, Panis C, et al. (2015) A multi-targeted approach to suppress tumor-promoting inflammation. Semin Cancer Biol 35 (Suppl):S151-S184.

Santen RJ, Yue W, and Wang JP (2015) Estrogen metabolites and breast cancer. Steroids 99 (Pt A):61-66.

Sas L, Lardon F, Vermeulen PB, Hauspy J, Van Dam P, Pauwels P, Dirix LY, and Van Laere SJ (2012) The interaction between ER and NFאB in resistance to endocrine therapy. Breast Cancer Res 14:212

Saugspier M, Dorn C, Thasler WE, Gehrig M, Heilmann J, and Hellerbrand C (2012) Hop bitter acids exhibit anti-fibrogenic effects on hepatic stellate cells in vitro. Exp Mol Pathol 92:222-228.

Saunders EJ and Saunders JA (1990) Drug therapy in pregnancy: the lessons of diethylstilbestrol, thalidomide, and bendectin. Health Care Women Int 11: 423-432.

Saw CL, Wu Q, Su ZY, Wang H, Yang Y, Xu X, Huang Y, Khor TO, and Kong AN (2013) Effects of natural phytochemicals in Angelica sinensis (Danggui) on Nrf2mediated gene expression of phase II drug metabolizing enzymes and antiinflammation. Biopharm Drug Dispos 34:303-311.

Schellenberg R (2001) Treatment for the premenstrual syndrome with agnus castus fruit extract: prospective, randomised, placebo controlled study. BMJ 322:134-137. Schellenberg R, Zimmermann C, Drewe J, Hoexter G, and Zahner C (2012) Dosedependent efficacy of the Vitex agnus castus extract Ze 440 in patients suffering from premenstrual syndrome. Phytomedicine 19:1325-1331.

Schierbeck LL, Rejnmark L, Tofteng CL, Stilgren L, Eiken P, Mosekilde L, Køber L, and Jensen JE (2012) Effect of hormone replacement therapy on 
cardiovascular events in recently postmenopausal women: randomised trial. BMJ 345:e6409.

Schinkovitz A, Dietz B, Deng S, Chen SN, Pro S, Lankin D, Nikolic D, van Breemen RB, Bolton JL, Farnsworth NR, et al. (2007) The stability of Z-ligustilide and its relevance for the biological evaluation of Angelica botanicals (Abstract). Planta Med 73:817.

Schinkovitz A, Pro SM, Main M, Chen SN, Jaki BU, Lankin DC, and Pauli GF (2008) Dynamic nature of the ligustilide complex. J Nat Prod 71:1604-1611.

Scott LM, Durant P, Leone-Kabler S, Wood CE, Register TC, Townsend A, and Cline JM (2008) Effects of prior oral contraceptive use and soy isoflavonoids on estrogen-metabolizing cytochrome P450 enzymes. J Steroid Biochem Mol Biol 112:179-185.

Seibel J, Molzberger AF, Hertrampf T, Laudenbach-Leschowski U, and Diel P (2009) Oral treatment with genistein reduces the expression of molecular and biochemica markers of inflammation in a rat model of chronic TNBS-induced colitis. Eur $J$ Nutr 48:213-220.

Seidlová-Wuttke D, Becker T, Christoffel V, Jarry H, and Wuttke W (2003) Silymarin is a selective estrogen receptor beta (ERbeta) agonist and has estrogenic effects in the metaphysis of the femur but no or antiestrogenic effects in the uterus of ovariectomized (ovx) rats. J Steroid Biochem Mol Biol 86:179-188.

Setchell KD, Clerici C, Lephart ED, Cole SJ, Heenan C, Castellani D, Wolfe BE, Nechemias-Zimmer L, Brown NM, Lund TD, et al. (2005) S-equol, a potent ligand for estrogen receptor beta, is the exclusive enantiomeric form of the soy isoflavone metabolite produced by human intestinal bacterial flora. Am J Clin Nutr 81: 1072-1079.

Shakeri F, Taavoni S, Goushegir A, and Haghani H (2015) Effectiveness of red clover in alleviating menopausal symptoms: a 12 -week randomized, controlled trial. Climacteric 18:568-573.

Shams T, Setia MS, Hemmings R, McCusker J, Sewitch M, and Ciampi A (2010) Efficacy of black cohosh-containing preparations on menopausal symptoms: a meta-analysis. Altern Ther Health Med 16:36-44.

Shanafelt TD, Barton DL, Adjei AA, and Loprinzi CL (2002) Pathophysiology and treatment of hot flashes. Mayo Clin Proc 77:1207-1218.

Shanle EK and Xu W (2010) Selectively targeting estrogen receptors for cancer treatment. Adv Drug Deliv Rev 62:1265-1276.

Sharma U and Stevermer JJ (2009) Bisphosphonate therapy: when not to monitor BMD. J Fam Pract 58:594-596.

Shen W and Stearns V (2009) Treatment strategies for hot flushes. Expert Opin Pharmacother 10:1133-1144.

Shimada T (2006) Xenobiotic-metabolizing enzymes involved in activation and detoxification of carcinogenic polycyclic aromatic hydrocarbons. Drug Metab Pharmacokinet 21:257-276.

Shimada Y, Takahashi M, Miyazawa N, Ohtani T, Abiru Y, Uchiyama S, and Hishigaki H (2011) Identification of two novel reductases involved in equol biosynthesis in Lactococcus strain 20-92. J Mol Microbiol Biotechnol 21: 160-172.

Shord SS, Shah K, and Lukose A (2009) Drug-botanical interactions: a review of the laboratory, animal, and human data for 8 common botanicals. Integr Cancer Ther 8:208-227.

Siegel RL, Miller KD, and Jemal A (2016) Cancer statistics, 2016. CA Cancer J Clin 66:7-30

Simmler C, Anderson JR, Gauthier L, Lankin DC, McAlpine JB, Chen SN, and Pauli GF (2015a) Metabolite Profiling and Classification of DNA-Authenticated Licorice Botanicals. J Nat Prod 78:2007-2022.

Simmler C, Chen SN, Anderson J, Lankin DC, Phansalkar R, Krause E, Dietz BM, Bolton JL, Nikolic D, van Breemen RB, et al. (2015b) Botanical integrity: the importance of the integration of chemical, biological, and botanical analyses, and the role of DNA barcoding. HerbalGram 106:56-58.

Simmler C, Hajirahimkhan A, Lankin DC, Bolton JL, Jones T, Soejarto DD, Chen SN, and Pauli GF (2013) Dynamic residual complexity of the isoliquiritigeninliquiritigenin interconversion during bioassay. J Agric Food Chem 61 2146-2157.

Sink R, Gobec S, Pečar S, and Zega A (2010) False positives in the early stages of drug discovery. Curr Med Chem 17:4231-4255.

Smith T, Lynch ME, Johnson J, Kawa K, Bauman H, and Blumenthal M (2015) Herbal dietary supplement sales in US increase $6.8 \%$ in 2014. Retail sales total more than $\$ 6.4$ billion in 11th consecutive year of growth. HerbalGram 107:52-59.

Snelten CS, Dietz B, and Bolton JL (2012) Modulation of estrogen chemical carcinogenesis by botanical supplements used for postmenopausal women's health Drug Discov Today Dis Mech 9:e47-e54.

Sprouse AA and van Breemen RB (2016) Pharmacokinetic interactions between drugs and botanical dietary supplements. Drug Metab Dispos 44:162-171.

Sreeja S, Anju VS, and Sreeja S (2010) In vitro estrogenic activities of fenugreek Trigonella foenum graecum seeds. Indian J Med Res 131:814-819.

Stanczyk FZ and Bhavnani BR (2014) Use of medroxyprogesterone acetate for hormone therapy in postmenopausal women: is it safe? J Steroid Biochem Mol Biol 142:30-38

Stärkel P, De Saeger C, Delire B, Magat J, Jordan B, Konda VR, Tripp ML, and Borbath I (2015) Tetrahydro iso-alpha acids and hexahydro iso-alpha acids from hops inhibit proliferation of human hepatocarcinoma cell lines and reduce diethylnitrosamine induced liver tumor formation in rats. Nutr Cancer 67: 748-760.

Strathmann J, Klimo K, Sauer SW, Okun JG, Prehn JH, and Gerhäuser C (2010) Xanthohumol-induced transient superoxide anion radical formation triggers cancer cells into apoptosis via a mitochondria-mediated mechanism. FASEB J 24: $2938-2950$

Su YW, Chiou WF, Chao SH, Lee MH, Chen CC, and Tsai YC (2011) Ligustilide prevents LPS-induced iNOS expression in RAW 264.7 macrophages by preventing ROS production and down-regulating the MAPK, NF-кB and AP-1 signaling pathways. Int Immunopharmacol 11:1166-1172.
Su ZY, Khor TO, Shu L, Lee JH, Saw CL, Wu TY, Huang Y, Suh N, Yang CS, Conney $\mathrm{AH}$, et al. (2013) Epigenetic reactivation of Nrf2 in murine prostate cancer TRAMP $\mathrm{C} 1$ cells by natural phytochemicals Z-ligustilide and Radix angelica sinensis via promoter CpG demethylation. Chem Res Toxicol 26:477-485.

Swedenborg E and Pongratz I (2010) AhR and ARNT modulate ER signaling. Toxicology 268:132-138.

Taavoni S, Nazem Ekbatani N, and Haghani H (2013) Valerian/lemon balm use for sleep disorders during menopause. Complement Ther Clin Pract 19:193-196.

Takemura H, Sakakibara H, Yamazaki S, and Shimoi K (2013) Breast cancer and flavonoids - a role in prevention. Curr Pharm Des 19:6125-6132.

Takahashi T, Takasuka N, Iigo M, Baba M, Nishino $\mathrm{H}$, Tsuda $\mathrm{H}$, and Okuyama T (2004) Isoliquiritigenin, a flavonoid from licorice, reduces prostaglandin E2 and nitric oxide, causes apoptosis, and suppresses aberrant crypt foci development. Cancer Sci 95:448-453.

Tamagno G (2009) Are changes of prolactin levels the effectors of vitex agnus castus beneficial effects on the pre-menstrual syndrome? Maturitas 63:369.

Tamir S, Eizenberg M, Somjen D, Stern N, Shelach R, Kaye A, and Vaya J (2000) Estrogenic and antiproliferative properties of glabridin from licorice in human breast cancer cells. Cancer Res 60:5704-5709.

Tang ZH, Li T, Tong YG, Chen XJ, Chen XP, Wang YT, and Lu JJ (2015) A systematic review of the anticancer properties of compounds isolated from licorice (Gancao). Planta Med 81:1670-1687.

Tanmahasamut P, Vichinsartvichai P, Rattanachaiyanont M, Techatraisak K, Dangrat C, and Sardod P (2015) Cimicifuga racemosa extract for relieving menopausal symptoms: a randomized controlled trial. Climacteric 18:79-85.

Taylor M (2012) Complementary and alternative medicine preparations used to treat symptoms of menopause. Menopausal Medicine 20:S1-S8.

Taylor M (2015) Complementary and alternative approaches to menopause. Endocrinol Metab Clin North Am 44:619-648.

Taylor CK, Levy RM, Elliott JC, and Burnett BP (2009) The effect of genistein aglycone on cancer and cancer risk: a review of in vitro, preclinical, and clinical studies. Nutr Rev 67:398-415.

Teelucksingh S, Mackie AD, Burt D, McIntyre MA, Brett L, and Edwards CR (1990) Potentiation of hydrocortisone activity in skin by glycyrrhetinic acid. Lancet $\mathbf{3 3 5}$ : 1060-1063

Teschke R, Schulze J, Schwarzenboeck A, Eickhoff A, and Frenzel C (2013) Herbal hepatotoxicity: suspected cases assessed for alternative causes. Eur J Gastroenterol Hepatol 25:1093-1098.

Thomas AJ, Ismail R, Taylor-Swanson L, Cray L, Schnall JG, Mitchell ES and Woods NF (2014) Effects of isoflavones and amino acid therapies for hot flashes and co-occurring symptoms during the menopausal transition and early postmenopause: a systematic review. Maturitas 78:263-276.

Thomas C and Gustafsson JA (2011) The different roles of ER subtypes in cancer biology and therapy. Nat Rev Cancer 11:597-608.

Thorne N, Auld DS, and Inglese J (2010) Apparent activity in high-throughput screening: origins of compound-dependent assay interference. Curr Opin Chem Biol 14:315-324.

Thorup AC, Lambert MN, Kahr HS, Bjerre M, and Jeppesen PB (2015) Intake of novel red clover supplementation for 12 weeks improves bone status in healthy menopausal women. Evid Based Complement Alternat Med DOI: 10.1155/2015/ 689138.

Tiong CT, Chen C, Zhang SJ, Li J, Soshilov A, Denison MS, Lee LS, Tam VH, Wong SP, Xu HE, et al. (2012) A novel prenylflavone restricts breast cancer cell growth through AhR-mediated destabilization of ER $\alpha$ protein. Carcinogenesis $\mathbf{3 3}$ 1089-1097.

Tolleson WH, Doerge DR, Churchwell MI, Marques MM, and Roberts DW (2002) Metabolism of biochanin A and formononetin by human liver microsomes in vitro. I Agric Food Chem 50:4783-4790.

Tsai JP, Hsiao PC, Yang SF, Hsieh SC, Bau DT, Ling CL, Pai CL, and Hsieh YH (2014) Licochalcone A suppresses migration and invasion of human hepatocellular carcinoma cells through downregulation of MKK4/JNK via NF-кB mediated urokinase plasminogen activator expression. PLoS One 9:e86537.

Turkyılmaz C, Onal E, Hirfanoglu IM, Turan O, Koç E, Ergenekon E, and Atalay Y (2011) The effect of galactagogue herbal tea on breast milk production and shortterm catch-up of birth weight in the first week of life. J Altern Complement Med 17 $139-142$

Ulbricht C, Costa D, Dam C, D'Auria D, Giese N, Isaac R, LeBlanc Y, Rusie E, Weissner W, and Windsor RC (2015) An evidence-based systematic review of kudzu (Pueraria lobata) by the Natural Standard Research Collaboration. J Diet Suppl 12:36-104

Valentová K, Buckiová D, Kren V, Peknicová J, Ulrichová J, and Simánek V (2006) The in vitro biological activity of Lepidium meyenii extracts. Cell Biol Toxicol 22: 91-99.

van Andel T, de Boer HJ, Barnes J, and Vandebroek I (2014) Medicinal plants used for menstrual disorders in Latin America, the Caribbean, sub-Saharan Africa, South and Southeast Asia and their uterine properties: a review. J Ethnopharmacol 155: 992-1000.

van Breemen RB (2015) Development of safe and effective botanical dietary supplements. J Med Chem 58:8360-8372.

van Breemen RB, Fong HH, and Farnsworth NR (2008) Ensuring the safety of botanical dietary supplements. Am J Clin Nutr 87:509S-513S.

van Breemen RB, Yuan Y, Banuvar S, Shulman LP, Qiu X, Alvarenga RF, Chen SN Dietz BM, Bolton JL, Pauli GF, et al. (2014) Pharmacokinetics of prenylated hop phenols in women following oral administration of a standardized extract of hops. Mol Nutr Food Res 58:1962-1969.

van der Velpen V, Geelen A, Schouten EG, Hollman PC, Afman LA, and van 't Vee P (2013) Estrogen receptor-mediated effects of isoflavone supplementation were not observed in whole-genome gene expression profiles of peripheral blood mononuclear cells in postmenopausal, equol-producing women. J Nutr 143: $774-780$. 
van Die MD, Burger HG, Teede HJ, and Bone KM (2009) Vitex agnus-castus (Chaste-Tree/ Berry) in the treatment of menopause-related complaints. J Altern Complement Med 15:853-862.

van Duursen MB, Smeets EE, Rijk JC, Nijmeijer SM, and van den Berg M (2013) Phytoestrogens in menopausal supplements induce ER-dependent cell proliferation and overcome breast cancer treatment in an in vitro breast cancer model. Toxicol Appl Pharmacol 269:132-140.

van Meeuwen JA, Korthagen N, de Jong PC, Piersma AH, and van den Berg M (2007) (Anti)estrogenic effects of phytochemicals on human primary mammary fibroblasts, MCF-7 cells and their co-culture. Toxicol Appl Pharmacol 221: $372-383$

van Meeuwen JA, Nijmeijer S, Mutarapat T, Ruchirawat S, de Jong PC, Piersma AH, and van den Berg M (2008) Aromatase inhibition by synthetic lactones and flavonoids in human placental microsomes and breast fibroblasts-a comparative study. Toxicol Appl Pharmacol 228:269-276.

Vanherweghem LJ (1998) Misuse of herbal remedies: the case of an outbreak of terminal renal failure in Belgium (Chinese herbs nephropathy). J Altern Complement Med 4:9-13.

Vanhoecke B, Derycke L, Van Marck V, Depypere H, De Keukeleire D, and Bracke M (2005) Antiinvasive effect of xanthohumol, a prenylated chalcone present in hops (Humulus lupulus L.) and beer. Int $J$ Cancer 117:889-895.

Vargas-Mendoza N, Madrigal-Santillán E, Morales-González A, Esquivel-Soto J, Esquivel-Chirino C, García-Luna Y González-Rubio M, Gayosso-de-Lucio JA, and Morales-González JA (2014) Hepatoprotective effect of silymarin. World $J$ Hepatol 6:144-149.

Vasileiou I, Katsargyris A, Theocharis S, and Giaginis C (2013) Current clinical status on the preventive effects of cranberry consumption against urinary tract infections. Nutr Res 33:595-607.

Vollmer G, Papke A, and Zierau O (2010) Treatment of menopausal symptoms by an extract from the roots of rhapontic rhubarb: the role of estrogen receptors. Chin Med 5:7.

Vona-Davis L and Rose DP (2013) The obesity-inflammation-eicosanoid axis in breast cancer. J Mammary Gland Biol Neoplasia 18:291-307.

Wagner H (2011) Synergy research: approaching a new generation of phytopharmaceuticals. Fitoterapia 82:34-37.

Wagner J, Jiang L, and Lehmann L (2008) Phytoestrogens modulate the expression of 17alpha-estradiol metabolizing enzymes in cultured MCF-7 cells. Adv Exp Med Biol 617:625-632.

Wang S, Dunlap TL, Howell CE, Mbachu OC, Rue EA, Chen SN, Pauli GF, Dietz $\mathrm{BM}$, and Bolton JL (2016) Hops extract and the AhR agonist, 6-prenylnaringenin increase P450 1A1 catalyzed estrogen metabolism. Chem Res Toxicol 29 1142-1150.

Wang KL, Hsia SM, Chan CJ, Chang FY, Huang CY, Bau DT, and Wang PS (2013) Inhibitory effects of isoliquiritigenin on the migration and invasion of human breast cancer cells. Expert Opin Ther Targets 17:337-349.

Wang W, Liu LQ, Higuchi CM, and Chen H (1998) Induction of NADPH:quinone reductase by dietary phytoestrogens in colonic Colo205 cells. Biochem Pharmacol 56:189-195.

Wang ZQ and Lou YJ (2004) Proliferation-stimulating effects of icaritin and desmethylicaritin in MCF-7 cells. Eur J Pharmacol 504:147-153.

Wang S, Penchala S, Prabhu S, Wang J, and Huang Y (2010) Molecular basis of traditional Chinese medicine in cancer chemoprevention. Curr Drug Discov Technol 7:67-75.

Wang X, Simpson ER, and Brown KA (2015b) Aromatase overexpression in dysfunctional adipose tissue links obesity to postmenopausal breast cancer. J Steroid Biochem Mol Biol 153:35-44.

Wang L, Yang R, Yuan B, Liu Y, and Liu C (2015a) The antiviral and antimicrobial activities of licorice, a widely-used Chinese herb. Acta Pharm Sin B 5 310-315

Warri A, Saarinen NM, Makela S, and Hilakivi-Clarke L (2008) The role of early life genistein exposures in modifying breast cancer risk. $\mathrm{Br} J$ Cancer 98 1485-1493.

Webb TE, Stromberg PC, Abou-Issa H, Curley RW Jr, and Moeschberger M (1992) Effect of dietary soybean and licorice on the male F344 rat: an integrated study of some parameters relevant to cancer chemoprevention. Nutr Cancer 18 $215-230$

Webster DE, He Y, Chen SN, Pauli GF, Farnsworth NR, and Wang ZJ (2011) Opioidergic mechanisms underlying the actions of Vitex agnus-castus L. Biochem Pharmacol 81:170-177.

Webster DE, Lu J, Chen SN, Farnsworth NR, and Wang ZJ (2006) Activation of the mu-opiate receptor by Vitex agnus-castus methanol extracts: implication for its use in PMS. J Ethnopharmacol 106:216-221.

Wei YK, Gamra I, Davenport A, Lester R, Zhao L, and Wei Y (2015) Genistein Induces cytochrome P450 1B1 gene expression and cell proliferation in human breast cancer MCF-7 Cells. J Environ Pathol Toxicol Oncol 34:153-159.

Weller MG (2012) A unifying review of bioassay-guided fractionation, effect-directed analysis and related techniques. Sensors (Basel) 12:9181-9209.

Wettasinghe M, Shahidi F, and Amarowicz R (2002) Identification and quantification of low molecular weight phenolic antioxidants in seeds of evening primrose (Oenothera biennis L.). J Agric Food Chem 50:1267-1271.

Wiegand H, Wagner AE, Boesch-Saadatmandi C, Kruse HP, Kulling S, and Rimbach G (2009) Effect of dietary genistein on Phase II and antioxidant enzymes in rat liver Cancer Genomics Proteomics 6:85-92.

Willenberg I, Meschede AK, Gueler F, Jang MS, Shushakova N, and Schebb NH (2015) Food Polyphenols Fail to Cause a Biologically Relevant Reduction of COX-2 Activity. PLoS One 10:e0139147.

Winterhoff H, Spengler B, Christoffel V, Butterweck V, and Löhning A (2003) Cimicifuga extract BNO 1055: reduction of hot flushes and hints on antidepressant activity. Maturitas 44 (Suppl 1):S51-S58.
Wober J, Möller F, Richter T, Unger C, Weigt C, Jandausch A, Zierau O, Rettenberger R, Kaszkin-Bettag M, and Vollmer G (2007) Activation of estrogen receptor-beta by a special extract of Rheum rhaponticum (ERr 731), its aglycones and structurally related compounds. J Steroid Biochem Mol Biol 107: 191-201.

Wolfender JL, Marti G, Thomas A, and Bertrand S (2015) Current approaches and challenges for the metabolite profiling of complex natural extracts. J Chromatogr A 1382:136-164.

Wong VC, Lim CE, Luo X, and Wong WS (2009) Current alternative and complementary therapies used in menopause. Gynecol Endocrinol 25:166-174.

Wong TY, Lin SM, Poon CH, and Leung LK (2014) The licorice flavonoid isoliquiritigenin reduces DNA-binding activity of AhR in MCF-7 cells. Chem Biol Interact 221:70-76.

Woo J, Lau E, Ho SC, Cheng F, Chan C, Chan AS, Haines CJ, Chan TY, Li M, and Sham A (2003) Comparison of Pueraria lobata with hormone replacement therapy in treating the adverse health consequences of menopause. Menopause 10: 352-361.

Wright AL, Zinn R, Hohensinn B, Konen LM, Beynon SB, Tan RP, Clark IA, Abdipranoto A, and Vissel B (2013) Neuroinflammation and neuronal loss precede A $\beta$ plaque deposition in the hAPP-J20 mouse model of Alzheimer's disease. PLoS One 8:e59586.

Wu YC and Hsieh CL (2011) Pharmacological effects of Radix Angelica Sinensis (Danggui) on cerebral infarction. Chin Med 6:32.

Wu C, Kim HK, van Wezel GP, and Choi YH (2015a) Metabolomics in the natural products field-a gateway to novel antibiotics. Drug Discov Today Technol 13: $11-17$.

Wu TY, Khor TO, Saw CL, Loh SC, Chen AI, Lim SS, Park JH, Cai L, and Kong AN (2011) Anti-inflammatory/Anti-oxidative stress activities and differential regulation of Nrf2-mediated genes by non-polar fractions of tea Chrysanthemum zawadskii and licorice Glycyrrhiza uralensis. AAPS J 13:1-13.

Wu KL, Rayner CK, Chuah SK, Changchien CS, Lu SN, Chiu YC, Chiu KW, and Lee CM (2008b) Effects of ginger on gastric emptying and motility in healthy humans. Eur J Gastroenterol Hepatol 20:436-440.

Wu Z, Uchi H, Morino-Koga S, Nakamura-Satomura A, Kita K, Shi W, and Furue M (2014) Z-Ligustilide inhibits benzo(a)pyrene-induced CYP1A1 upregulation in cultured human keratinocytes via ROS-dependent Nrf2 activation. Exp Dermatol 23:260-265.

Wu WY, Wu YY, Huang H, He C, Li WZ, Wang HL, Chen HQ, and Yin YY (2015b) Biochanin A attenuates LPS-induced pro-inflammatory responses and inhibits the activation of the MAPK pathway in BV2 microglial cells. Int $\mathrm{J}$ Mol Med 35: 391-398.

Wu AH, Yu MC, Tseng CC, and Pike MC (2008a) Epidemiology of soy exposures and breast cancer risk. Br J Cancer 98:9-14.

Wuttke W, Jarry H, Christoffel V, Spengler B, and Seidlová-Wuttke D (2003) Chaste tree (Vitex agnus-castus)-pharmacology and clinical indications. Phytomedicine 10:348-357.

Wuttke W, Jarry H, Haunschild J, Stecher G, Schuh M, and Seidlova-Wuttke D (2014) The non-estrogenic alternative for the treatment of climacteric complaints: Black cohosh (Cimicifuga or Actaea racemosa). J Steroid Biochem Mol Biol 139: 302-310.

Wyns C, van Steendam K, Vanhoecke B, Deforce D, Bracke M, and Heyerick A (2012) Prenylated chalcone xanthohumol associates with histones in breast cancer cells-a novel target identified by a monoclonal antibody. Mol Nutr Food Res 56:1688-1696.

Xiao HH, Fung CY, Mok SK, Wong KC, Ho MX, Wang XL, Yao XS, and Wong MS (2014) Flavonoids from Herba epimedii selectively activate estrogen receptor alpha $(\mathrm{ER} \alpha)$ and stimulate ER-dependent osteoblastic functions in UMR-106 cells. $J$ Steroid Biochem Mol Biol 143:141-151.

Yadav UC and Baquer NZ (2014) Pharmacological effects of Trigonella foenumgraecum L. in health and disease. Pharm Biol 52:243-254.

Yager JD (2015) Mechanisms of estrogen carcinogenesis: The role of E2/E1-quinone metabolites suggests new approaches to preventive intervention-A review. Ste roids 99 ( $\mathrm{Pt} \mathrm{A}): 56-60$.

Yamamoto S, Sobue T, Kobayashi M, Sasaki S, and Tsugane S; Japan Public Health Center-Based Prospective Study on Cancer Cardiovascular Diseases Group (2003) Soy, isoflavones, and breast cancer risk in Japan. J Natl Cancer Inst 95:906-913.

Yan FF, Liu Y, Liu YF, and Zhao YX (2008) Herba Epimedii water extract elevates estrogen level and improves lipid metabolism in postmenopausal women. Phytother Res 22:1224-1228.

Yang X, Belosay A, Hartman JA, Song H, Zhang Y, Wang W, Doerge DR and Helferich WG (2015) Dietary soy isoflavones increase metastasis to lungs in an experimental model of breast cancer with bone micro-tumors. Clin Exp Metastasis 32:323-333.

Yang Z, Kulkarni K, Zhu W, and Hu M (2012) Bioavailability and pharmacokinetics of genistein: mechanistic studies on its ADME. Anticancer Agents Med Chem 12: $1264-1280$

Yarnell E (2002) Botanical medicines for the urinary tract. World $J$ Urol 20:285-293. Ye L, Chan MY, and Leung LK (2009a) The soy isoflavone genistein induces estrogen synthesis in an extragonadal pathway. Mol Cell Endocrinol 302:73-80.

Ye L, Gho WM, Chan FL, Chen S, and Leung LK (2009b) Dietary administration of the licorice flavonoid isoliquiritigenin deters the growth of MCF-7 cells overexpressing aromatase. Int $J$ Cancer 124:1028-1036.

Yoshimaru T, Komatsu M, Tashiro E, Imoto M, Osada H, Miyoshi Y, Honda J, Sasa M, and Katagiri T (2014) Xanthohumol suppresses oestrogen-signalling in breast cancer through the inhibition of BIG3-PHB2 interactions. Sci Rep 4:7355.

Yu JY, Ha JY, Kim KM, Jung YS, Jung JC, and Oh S (2015) Anti-Inflammatory activities of licorice extract and its active compounds, glycyrrhizic acid, liquiritin and liquiritigenin, in BV2 cells and mice liver. Molecules 20:13041-13054. 
Zadoyan G and Fuhr U (2012) Phenotyping studies to assess the effects of phytopharmaceuticals on in vivo activity of main human cytochrome p450 enzymes. Planta Med 78:1428-1457.

Zahradnik HP, Hanjalic-Beck A, and Groth K (2010) Nonsteroidal anti-inflammatory drugs and hormonal contraceptives for pain relief from dysmenorrhea: a review. Contraception 81:185-196.

Zamani M, Neghab N, and Torabian S (2012) Therapeutic effect of Vitex agnus castus in patients with premenstrual syndrome. Acta Med Iran 50:101-106.

Zanoli P and Zavatti M (2008) Pharmacognostic and pharmacological profile of Humulus lupulus L. J Ethnopharmacol 116:383-396.

Zapantis A, Steinberg JG, and Schilit L (2012) Use of herbals as galactagogues. $J$ Pharm Pract 25:222-231.

Zhang D, Ren Y, Dai S, Liu W, and Li G (2009) [Isoflavones from vines of Pueraria lobata]. Zhongguo Zhong Yao Za Zhi 34:3217-3220.
Zhang CZ, Wang SX, Zhang Y, Chen JP, and Liang XM (2005) In vitro estrogenic activities of Chinese medicinal plants traditionally used for the management of menopausal symptoms. J Ethnopharmacol 98:295-300.

Zhang Y, Yu L, Ao M, and Jin W (2006) Effect of ethanol extract of Lepidium meyenii Walp. on osteoporosis in ovariectomized rat. J Ethnopharmacol 105:274-279.

Zhao H, Zhang X, Chen X, Li Y, Ke Z, Tang T, Chai H, Guo AM, Chen H, and Yang J (2014) Isoliquiritigenin, a flavonoid from licorice, blocks M2 macrophage polarization in colitis-associated tumorigenesis through downregulating PGE2 and IL-6. Toxicol Appl Pharmacol 279:311-321.

Ziegler RG, Fuhrman BJ, Moore SC, and Matthews CE (2015) Epidemiologic studies of estrogen metabolism and breast cancer. Steroids 99 (Pt A):67-75.

Zuppa AA, Sindico P, Orchi C, Carducci C, Cardiello V, and Romagnoli C (2010) Safety and efficacy of galactogogues: substances that induce, maintain and increase breast milk production. J Pharm Pharm Sci 13:162-174. 